\title{
Transport impacts on atmosphere and climate: Metrics
}

\author{
J.S. Fuglestvedt ${ }^{a}$, K.P. Shine ${ }^{b, *}$, T. Berntsen ${ }^{a}$, J. Cook $^{b}$, D.S. Lee ${ }^{c}$, A. Stenke $^{\text {d }}$, R.B. Skeie ${ }^{a}$, \\ G.J.M. Velders ${ }^{\text {, }}$ I.A. Waitz ${ }^{\mathrm{f}}$ \\ ${ }^{a}$ CICERO-Center for International Climate and Environmental Research-Oslo, P.O. Box 1129, Blindern, 0318 Oslo, Norway \\ ${ }^{\mathrm{b}}$ Department of Meteorology, University of Reading, Earley Gate, Reading RG6 6BB, UK \\ ${ }^{\mathrm{c}}$ Dalton Research Institute, Department of Environmental and Geographical Sciences, Manchester Metropolitan University, \\ John Dalton Building, Chester Street, Manchester M1 5GD, UK \\ ${ }^{\mathrm{d}}$ DLR, Institut für Physik der Atmosphäre, Oberpfaffenhofen, D-82234 Wessling, Germany \\ e Netherlands Environmental Assessment Agency, P.O. Box 303, 3720 AH Bilthoven, The Netherlands \\ ${ }^{\mathrm{f}}$ Department of Aeronautics and Astronautics, Massachusetts Institute of Technology, Cambridge, MA 02139, USA
}

\section{A R T I C L E I N F O}

\section{Article history:}

Received 18 July 2008

Received in revised form

15 April 2009

Accepted 16 April 2009

\section{Keywords:}

Transport

Climate change

Global warming potential

GWP

Global Temperature Change Potential (GTP) Radiative forcing

\begin{abstract}
A B S T R A C T
The transport sector emits a wide variety of gases and aerosols, with distinctly different characteristics which influence climate directly and indirectly via chemical and physical processes. Tools that allow these emissions to be placed on some kind of common scale in terms of their impact on climate have a number of possible uses such as: in agreements and emission trading schemes; when considering potential trade-offs between changes in emissions resulting from technological or operational developments; and/or for comparing the impact of different environmental impacts of transport activities. Many of the non- $\mathrm{CO}_{2}$ emissions from the transport sector are short-lived substances, not currently covered by the Kyoto Protocol. There are formidable difficulties in developing metrics and these are particularly acute for such short-lived species. One difficulty concerns the choice of an appropriate structure for the metric (which may depend on, for example, the design of any climate policy it is intended to serve) and the associated value judgements on the appropriate time periods to consider; these choices affect the perception of the relative importance of short- and long-lived species. A second difficulty is the quantification of input parameters (due to underlying uncertainty in atmospheric processes). In addition, for some transport-related emissions, the values of metrics (unlike the gases included in the Kyoto Protocol) depend on where and when the emissions are introduced into the atmosphere - both the regional distribution and, for aircraft, the distribution as a function of altitude, are important.

In this assessment of such metrics, we present Global Warming Potentials (GWPs) as these have traditionally been used in the implementation of climate policy. We also present Global Temperature Change Potentials (GTPs) as an alternative metric, as this, or a similar metric may be more appropriate for use in some circumstances. We use radiative forcings and lifetimes from the literature to derive GWPs and GTPs for the main transport-related emissions, and discuss the uncertainties in these estimates. We find large variations in metric (GWP and GTP) values for $\mathrm{NO}_{x}$, mainly due to the dependence on location of emissions but also because of inter-model differences and differences in experimental design. For aerosols we give only global-mean values due to an inconsistent picture amongst available studies regarding regional dependence. The uncertainty in the presented metric values reflects the current state of understanding; the ranking of the various components with respect to our confidence in the given metric values is also given. While the focus is mostly on metrics for comparing the climate impact of emissions, many of the issues are equally relevant for stratospheric ozone depletion metrics, which are also discussed.
\end{abstract}

(c) 2009 Elsevier Ltd. All rights reserved.

\footnotetext{
* Corresponding author. Tel.: +44 118378 8405; fax: +44 1183788905.

E-mail address: k.p.shine@reading.ac.uk (K.P. Shine).
}

\section{Introduction}

Transportation is an important contributor to global emissions of many different gases and aerosols that can have an impact on climate and stratospheric ozone, either directly or indirectly (e.g. Eyring et al., 2005; Fuglestvedt et al., 2008; Kahn Ribeiro et al., 
2007). In 2000, the global transport sector was the largest source of man-made emissions of oxides of nitrogen $\left(\mathrm{NO}_{x}\right)$ (estimated to be $37 \%$ of the total anthropogenic emissions of $\mathrm{NO}_{x}$ ) and was also a major contributor of fossil-fuel $\mathrm{CO}_{2}(21 \%)$, volatile organic carbon (VOC) (19\%), CO (18\%) and black carbon (BC) (14\%) (Fuglestvedt et al., 2008); in addition, mobile air conditioning is the dominant source of emissions of HFC-134a (IPCC, 2005). The contributions of the different sectors of transport are discussed in detail in the accompanying assessments of aviation (Lee et al., 2010), shipping (Eyring et al., 2010) and land transport (Uherek et al., 2010).

The emissions from transportation have a wide range in their atmospheric lifetimes (which influences the overall concentrations and spatial distributions) and in their abilities, per unit change in atmospheric concentration, to affect climate or ozone. Despite the widely varying characteristics of the emitted substances, there is often a requirement to place their impacts on a common scale, to allow some kind of direct comparison, both across substances emitted and across sectors/sources. Here we use the word "metric" to refer to methods and tools which attempt to achieve this aim.

Such metrics can be used in a legislative framework, such as in the Kyoto Protocol (see below) which places emissions of a sub-set of greenhouse gases onto a " $\mathrm{CO}_{2}$-equivalent" scale. Ideally, the same equivalent $\mathrm{CO}_{2}$ emissions should produce the same climate effect regardless of their composition. The metrics can be used in an industrial or policymaking setting - for example to assess the net climate impact of technological, policy or operational changes of a mode of transport. Ultimately they could also be used to compare climate and non-climate impacts (such as noise or air quality) on some kind of common scale (e.g. monetary).

There are a number of important assumptions that underlie our discussion here. The first is that any metric that is used for these purposes should be transparent and relatively easy to apply. This is because the metric needs to be used by non-specialists without expert input at the point of use. An alternative might be to employ more sophisticated models (see Section 3) which, for example, include possible scenarios of future emissions. Such models are certainly invaluable and may, in the future, come to replace the metrics proposed here. However, it would be challenging to reach agreement on a single such model for use in the legislative framework of multi-gas agreements, such as the Kyoto and Montreal Protocols. ${ }^{1}$ As discussed below, in these Protocols, the relative weighting of the importance of different gases is represented by a single-valued metric. Such metrics have been successful in the sense that, despite any shortcomings, they have been easily and widely applied, and have not been perceived to be controversial amongst the user community. The formulation of these singlevalued metrics can also be capable of illustrating some of the basic physics of the atmospheric processes that may not be readily apparent in "black-box" usage of more sophisticated models.

A second assumption is that the presence of uncertainties is not itself a reason for refraining from providing values for metrics. If specialists in climate and ozone science do not deliver metric values, then the user community might either use inappropriate measures for comparing emissions, or develop metrics which do not represent the current state of understanding.

\footnotetext{
${ }^{1}$ Of course, future climate change agreements may adopt different types of multi-gas principles. Instead of a basket of gases, a multi-gas approach may also be achieved through a set of single-gas agreements or several different baskets of components. In that case metrics would still be needed to compare the total reductions that the individual agreements require. Even in the case of a one-gas policy (e.g. a $\mathrm{CO}_{2}$-only), metrics would be needed to motivate a decision to pursue such a single-gas policy. In both cases, the use of metrics would be lifted up from the implementation level to the design level in climate policy.
}

Nevertheless, as will become clear in this assessment, there is no single perfect metric. The methodological framework for metrics requires simplifications to complex atmospheric and climatic processes to be made, as well as value-laden decisions on, for example, the timescales that are to be considered. In addition, for many of the emissions considered here, there is a substantial underlying uncertainty in quantifying their impact, such that the values for some metrics may prove to be quite volatile as understanding increases. The users of metrics need to be aware of the simplifications and uncertainties when they are applied. Indeed, the metrics themselves can serve as an important tool in communicating the state of knowledge and overall uncertainty. Finally, the nature of the metric depends on the nature of the problem being addressed - different climate policy goals may lead to different conclusions about what are the most suitable metrics with which to implement that policy.

It is important to stress that the metrics proposed here do not define the policy - they are tools that enable implementation of the multi-gas policy. A policy which defines the overall future emission paths of climate or ozone-depleting gases, may seek to avoid some future amount of warming or ozone depletion. More sophisticated models will be required to define these total emission paths; the metrics will then be used to allow signatories to any agreements to decide how they will implement their responsibilities (i.e. which emissions they choose to abate).

The current situation in terms of the legislative use of metrics in climate science can be illustrated by a brief discussion of their application in the Kyoto Protocol to the United Nations Framework Convention on Climate Change (UNFCCC) (www.unfccc.int). Parties to the Kyoto Protocol are required to meet their commitments in terms of $\mathrm{CO}_{2}$-equivalent emissions of a group of greenhouse gases carbon dioxide, nitrous oxide, methane, the hydrofluorocarbons, the perfluorocarbons and sulphur hexafluoride. The emissions of these gases are placed on a common $\mathrm{CO}_{2}$-equivalent scale using the GWP (see Section 4 for details), that measures the radiative forcing $(\Delta F)$ of a pulse emission of a gas, integrated over 100 years, relative to the radiative forcing of a pulse emission of carbon dioxide integrated over 100 years, for some background atmospheric state (although the choice of this background state is not always made explicit - see Section 5.3). The Protocol uses values of GWP specified in the Second Assessment Report of the Intergovernmental Panel on Climate Change (IPCC, 1996) despite the fact that later assessments have revised (and expanded) the tables reporting values for GWPs.

The implementation of the Kyoto Protocol, and the use of the 100 -year GWP, can be used to focus on a number of issues in metric design and use. Why is the Protocol limited to a particular sub-set of emissions, rather than a wider set? This is particularly important for the transport sector where the vast majority of non- $\mathrm{CO}_{2}$ climate emissions are of substances not included in the Kyoto Protocol, some causing warming and some causing cooling. ${ }^{2}$ Why is the GWP chosen, as it quantifies a somewhat abstract characteristic of the climate effect of emissions (time-integrated radiative forcing)? Could some other measure (such as temperature change, sea-level change, economic damage) be used instead? What kind of equivalence does GWP express? Why is 100 years chosen as a time horizon? Does the cost-effectiveness of emission controls, or a costbenefit analysis, change if some other metric or parameter choice is

\footnotetext{
2 As discussed by Skodvin and Fuglestvedt (1997) the UNFCCC does not cover aerosols since its Article 3.3 links the principle of comprehensiveness to the term "greenhouse gases", defined as "...those gaseous constituents of the atmosphere, both natural and anthropogenic, that absorb and re-emit infrared radiation" (Article 1.5).
} 
made? And how would advances in understanding, which change the recommended values of the GWPs, affect how the Protocol is implemented?

Similar issues are also apparent in the application of Ozone Depletion Potentials (which formed a model for the use of GWPs) within the United Nations' Montreal Protocol on Substances that Deplete the Ozone Layer (ozone.unep.org).

Our assessment builds on previous assessments of climate and ozone metrics. These include general assessments under the auspices of the United Nations, such as by IPCC (IPCC, 1990, 1992, 1995, 1996, 1999, 2001, 2007) ${ }^{3}$ and WMO/UNEP Scientific Assessments of Ozone Depletion (WMO, 1999, 2003, 2007), as well as discussions and reviews in the wider science literature. For the most part, these have not focused on specific sources or sectors. There are relatively few discussions targeted specifically at metrics for transport, and the majority of these concern aircraft emissions (Berntsen and Fuglestvedt, 2008; Forster et al., 2006, 2007b; Forster and Rogers, 2008; Fuglestvedt et al., 2008; IPCC, 1999; Marais et al., 2008; Svensson et al., 2004; Wit et al., 2005; Wuebbles et al., 2008).

This assessment will first illustrate some fundamental concepts that determine the relevant timescales of the response of both atmospheric concentrations and the climate system to changes in emissions (Section 2), discuss the nature of the tools available to evaluate the impact of climate emissions (Section 3) and discuss how the use of emission metrics depends on the application (Section 4). Section 5 presents the climate metrics $\Delta F$, GWP and GTP and the practicalities and difficulties in their application. Section 6 will then focus more specifically on transport emissions and the difficulties in quantifying their climate impact. Section 7 will present metric values for transport-related emissions and assess them for their quality and robustness. For the most part, the discussion will focus on climate metrics, but Section 8 will discuss stratospheric ozone depletion metrics in the context of the transport sector. Finally, Section 9 will present conclusions regarding the status of our current understanding of the calculation and use of metrics, and discuss prospects for future improvements.

Note that the focus here will be on providing metrics to measure and intercompare the impact of emissions from the operation of vehicles in each transport sector. We do not present a methodology for the total environmental (or even climate) impact of a particular transport activity, including the effects of construction, the extraction and production of the fuel supply, operation and decommissioning (life-cycle analyses); nevertheless, the metrics discussed here can contribute to more holistic assessments.

\section{A general perspective}

Fig. 1 shows a standard cause and effect chain from emissions through physical changes in climate to ecological, health, or economic damage. ${ }^{4}$ In general, as one moves down the chain, the parameters become more relevant to society, but, at the same time, they become more difficult to quantify. In this section we illustrate the first steps in this chain for a simplified example, with all quantities taken to be global averages. Note that neither Fig. 1 nor the simplified example here, attempt to represent the many complex feedbacks that could exist in the climate system; changes in any of the boxes in Fig. 1 have the potential to impact on the processes in all the other boxes (for example, changes in

\footnotetext{
${ }^{3}$ IPCC also recently convened an expert group on the science of alternative metrics - the summary is reported in IPCC (2009).

4 This term is used here, following convention, although it is recognised that emissions can also have positive impacts.
}

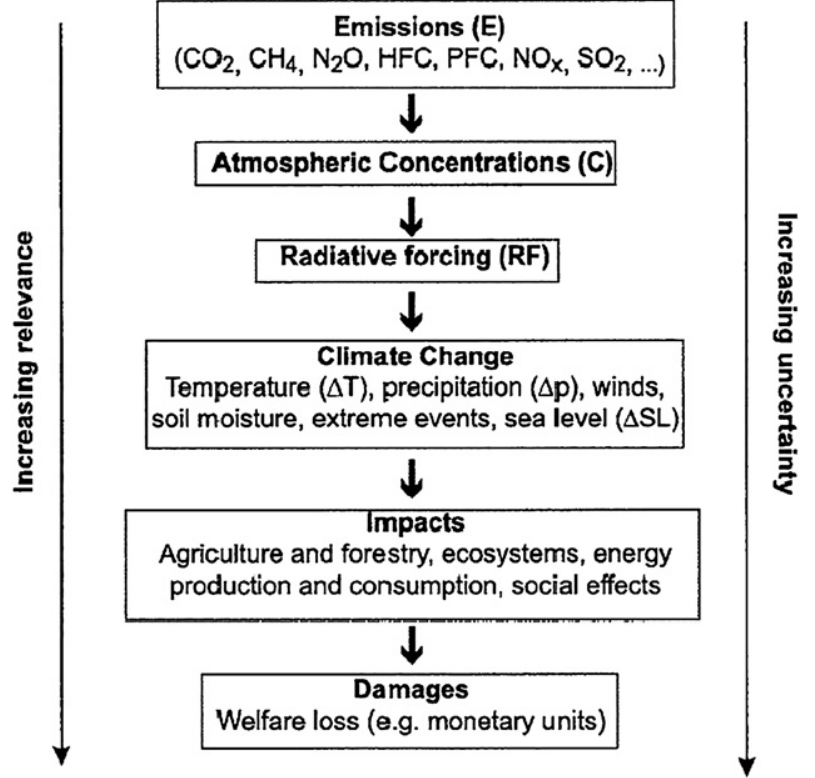

Fig. 1. Cause and effect chain of the potential climate effect of emissions (from Fuglestvedt et al., 2003).

temperature can impact on $\mathrm{CO}_{2}$ emissions or the rate of reactions that destroy methane).

Consider a gas or aerosol component, whose removal from the atmosphere can be represented by a simple exponential decay, with lifetime $\alpha$. We assume that there are no natural emissions or background concentrations of this gas, but the analysis can be easily modified to consider perturbations from this natural background. We take the emission sources of this gas to be $S(t)$ (in units of, say, $\mathrm{kg}$ year $^{-1}$ ), where $t$ is time. If $X(t)$ is the time-dependent abundance of this gas (which could be presented as a global abundance in the atmosphere in $\mathrm{kg}$, or as a mixing ratio, etc.), then its rate of change is determined by the balance between emissions and sinks. In the absence of sources, the abundance will be represented as $X(t)=X_{o} \exp (-t / \alpha)$ where $X_{o}$ is the abundance at the initial time; the rate of change of $X$ is then $-X(t) / \alpha$. Hence, the net rate of change, including sources, is

$\frac{\mathrm{d} X(t)}{\mathrm{d} t}=S(t)-\frac{X(t)}{\alpha}$.

For a case with $S=$ constant from time $t=0$, the solution to (1) is

$X(t)=\alpha S[1-\exp (-t / \alpha)]$.

This shows that the timescale for the approach of $X$ to equilibrium is determined by the lifetime, and the equilibrium abundance is equal to the emissions multiplied by this lifetime.

The radiative forcing, $\Delta F$, can be determined from the change in concentration. We assume the simplest case whereby the forcing is linearly proportional to the abundance. If $A$ is the specific forcing (i.e. the forcing per unit change in abundance), then

$\Delta F(t)=A X(t)$.

The radiative forcing drives a surface temperature change $\Delta T(t)$. The simplest representation of the climate system (see, e.g. Hartmann, 1996) assumes that it can be represented as single component with heat capacity $C$ (in such a model, this could represent, for example, the heat capacity of the mixed layer of the ocean) so that 
$C \frac{\mathrm{d} \Delta T(t)}{\mathrm{d} t}=\Delta F(t)-\frac{\Delta T(t)}{\lambda}$.

Here $\lambda$ is a climate sensitivity, which indicates the equilibrium surface temperature change per unit radiative forcing; its reciprocal gives the change in radiative balance following a unit change in temperature, and hence it characterises the strength of the response necessary to re-establish equilibrium with any imposed forcing. Low values of $\lambda$ indicate an insensitive climate and high values indicate a sensitive climate. The value of $\lambda$ is uncertain, due to poorly understood feedbacks (such as those relating to clouds). IPCC (2007) reports that $\lambda$ is likely to lie in the range from about 0.54 to $1.2 \mathrm{~K}\left(\mathrm{Wm}^{-2}\right)^{-1}$; IPCC (2007) also reports that $\lambda$ is very unlikely to be less than the lower limit, but values substantially higher than the upper limit cannot be ruled out; in addition, as will be discussed in Section 5.2, the value of $\lambda$ may vary among different climate change mechanisms.

General solutions to Equation (4) are available (e.g. Hartmann, 1996) but for a hypothetical case of a constant radiative forcing imposed at time $t=0$, the solution is

$\Delta T(t)=\lambda \Delta F[1-\exp (-t / \lambda C)]$

This illustrates two important points. Firstly, the timescale for the climate system to respond to a perturbation is given by the product $\lambda C$, and is typically of order of decades. Second, the equilibrium response to the constant forcing is given by $\Delta T_{\mathrm{eq}}=\lambda \Delta F$.

The above analysis shows that there are two important timescales. The first is the lifetime of gases or aerosols in the atmosphere (or for $\mathrm{CO}_{2}$, the lifetime of the perturbation), which is dictated by chemical reactions or other removal mechanisms (such as wet or dry deposition). The second is the timescale of the response of the climate system to a radiative forcing, which is determined by the product of the climate sensitivity and the heat capacity of the climate system. An important point here is that if there were to be a pulse emission of gas into the atmosphere, then the climate response will depend on the relative size of the two timescales. If the gas is short-lived, compared to the timescale of the climate system, then the climate system will have insufficient time to fully respond before the pulse has disappeared $^{5}$; by contrast, if the gas is very long-lived, the climate system will be able to fully respond to the impact of an emission. This dependence on the two timescales will be illustrated in the discussion of the Global Temperature Change Potential in Section 5.4 (see especially Equation (7)). More extended discussions of the relationship between these timescales are available (e.g. Schneider and Thompson, 1981; Shine et al., 2005b; Wigley et al., 2005).

Equation (5) can be used as a basis to extend the calculation to, for example, changes in global-mean sea level and, if appropriate functions are available, the economic or ecosystem damage, resulting from the climate change (Fig. 1). However, damage, and changes in parameters such as precipitation or extreme events, are less amenable to a global-mean analysis and calculations need to be performed at smaller spatial scales (Section 3).

\section{Types of tools available for evaluating the impacts of transport emissions}

Ultimately the accurate evaluation of the impacts of emissions on climate or ozone, requires state-of-the-art Earth System Models,

\footnotetext{
${ }^{5}$ Note that sustained emissions of short-lived species can exert a significant impact on climate, but this influence is still muted compared to the effect that a longer-lived species, with the same emissions and same radiative properties, would exert.
}

that include detailed representations of, and interactions among, the atmosphere, its chemical composition, the oceans, biosphere, cryosphere, etc. These models encapsulate our understanding, at least on larger scales, of the important physical, chemical and biological processes. However, they are not useful in directly providing metrics for, for example, policymaking for several reasons. They require very large computer resources and considerable expertise to perform calculations and to diagnose results from the large amount of output that they produce. Hence, there is a limit on the number of different cases (for example, scenarios of future emissions) that can be considered. Because of uncertainties in the basic physical processes, it is not possible to rely on results from one such model, and hence results from an ensemble of different models are required.

Nevertheless, it is results from these more sophisticated models which form the basis for the parameter choices in the simpler models that are in widespread use. There is a hierarchy of simpler models available. For example, within the IPCC process, the socalled Upwelling-Diffusion Energy Balance Models (UD-EBMs) have been widely used to explore the dependence on scenarios (e.g. Wigley and Raper, 2001). These models are tuned to reproduce the response of the much more sophisticated coupled ocean-atmosphere general circulation models (GCMs). In addition to the modest computer resources required by these models, one important attraction is that poorly-known parameters (such as the climate sensitivity parameter - see Section 2) can be altered systematically to examine the influence of uncertainty in model results. It is not straightforward to perform such systematic tests within an individual GCM, although methodologies to search the parameter space of uncertainty within individual GCMs are being developed (e.g. Murphy et al., 2004; Stainforth et al., 2005).

These simplified models are also used as components of integrated-assessment models, which include, for example, additional economic components and components estimating other impacts of climate change; these models have been used to compute costeffective emission strategies given some constraint on future climate change (e.g. den Elzen and Meinshausen, 2006; van Vuuren et al., 2006).

An even more simplified modelling framework has commonly been adopted in studies of past and future climate change - the socalled linear response (or impulse-response) model. This framework can be used to model both the response of concentrations to emissions, and the response of climate to the resulting forcings and it has been widely applied in studies of the impact of the transport sector (Grewe and Stenke, 2008; Lee et al., 2006; Marais et al., 2008; Sausen and Schumann, 2000). These response models can use either the type of simplified models discussed in Section 2, or use fits to results generated by GCMs or UD-EBMs. The calculation for an individual pulse of gas emitted can be amenable to analytical solution, but generally such models integrate over many pulses, and do so numerically. We are unaware of any clear demonstration that these models are inferior in performance to the UD-EBMs.

The hierarchy of models described above largely refers to understanding and modelling the effects of emissions on climate change. A similar hierarchy exists for stratospheric ozone depletion (see e.g. WMO, 2007), although more use has been made of twodimensional (latitude-altitude) models. Such models can include the interactions between chemistry, radiative processes and atmospheric dynamical processes. More recently, there has been increasing use of 3-D chemical transport models (CTMs) (which include detailed representations of chemical processes, but use pre-specified distributions of winds, to advect the chemicals, and temperatures, to determine the rate of chemical reactions) and 3-D coupled chemistry-climate models (CCMs). CCMs include full representations of dynamical, radiative, and chemical processes in 
the atmosphere and their interactions. In particular, CCMs include feedbacks of the chemical tendencies on the dynamics, and hence the transport of chemicals. This feedback is a major difference between CCMs and CTMs, and is required to simulate the evolution of ozone in a changing climate.

Simplified models for ozone depletion and climate impacts have not, as yet, been favoured to provide the emission metrics used for comparing emissions of climate or ozone-depleting gases within policymaking. The Global Warming Potential (Section 5) and the Ozone Depletion Potential (Section 8) are simpler and more transparent formulations, albeit still requiring much input from more complex models for the parameters that drive them. It is possible that in future there will be a move away from the simple metrics, towards an explicit use of the more complex models for use in policy implementation.

It is important to stress that in the case of poorly understood effects of transport (or any other emissions), results from the more complex climate models should not necessarily be regarded as any more reliable than those from simpler models. As an example, understanding of the radiative forcing due to aviation-induced cirrus (Forster et al., 2007a; Sausen et al., 2005) is very low. Methods to include such effects within GCMs are in their infancy, and there is little immediate prospect of rigorous evaluation of those methods using observations. In these circumstances, the simpler models play an important role, in allowing order-ofmagnitude assessments of the possible impacts of these poorly understood processes, and help motivate calculations using the more complex models. They also play an important role in assessing the impact of uncertainties in input or model parameters, as they allow for a more comprehensive searching of parameter space. Such models are also convenient tools for the evaluation of the effects of many components together $\left(\mathrm{CO}_{2}\right.$, sulphate, $\mathrm{O}_{3}$, cirrus etc) and show how they interact with the timescale of the climate system and contribute to the development in net temperature effect; such an evaluation, whilst possible with a GCM, would be extremely time consuming.

\section{Use of emissions metrics and their dependence on application}

\subsection{Dependence on policy context}

Adding together the climate impact of emissions of species that have different characteristics is somewhat analogous to calculating an "equivalence" for a mixture of apples and oranges (Tol et al., 2008). There is no single unique way that this can be done. For anyone wanting to transport fruits one might add apples and oranges by their weight (or volume). This is not because weight is the only attribute that makes an apple an apple and an orange an orange. Rather, this is because weight is the main thing that matters in freight transport. Similarly, a nutritionist might add apples and oranges by their nutrient content. A grocer might add apples and oranges by their prices. Thus, the metric of aggregation depends on the purpose of aggregation. In the present context, an example might be comparing emissions from shipping. $\mathrm{ACO}_{2}$ perturbation is long-lived (of order decades/centuries) and so has a persistent warming impact and spreads globally. Sulphur emissions, by contrast, are short-lived (of order days), cause cooling and are largely confined to the hemisphere in which they are emitted. This immediately raises issues of comparing short-lived versus longlived species, warming versus cooling agents, and in broader environmental terms, comparing the distinct acidification effects of $\mathrm{CO}_{2}$ and sulphur, and the health impacts of particulate matter arising from the sulphur.
The objective of the 1992 United Nations Framework Convention on Climate Change (UNFCCC) (www.unfccc.int) is the "stabilization of greenhouse gas concentrations in the atmosphere at a level that would prevent dangerous anthropogenic interference with the climate system"; it further states that measures to achieve this objective should be "cost-effective". The decision as to what constitutes a "dangerous anthropogenic interference with the climate system" ultimately involves value judgements and thus cannot be determined without a collaborative process involving policymakers and scientists. However, once a common goal has been agreed upon (e.g. the European Union's goal of restricting the global temperature increase to $2{ }^{\circ} \mathrm{C}$ above pre-industrial levels (www.europa.eu/bulletin/en/200503/i1010.htm)), a metric can be designed based on scientific methods to assess different mixes of emissions for achieving the goal. In a cost-effective framework, the environmental goal of the policy is specified essentially independent of the costs incurred; the emission metrics act as tools to assess how the fixed target may be met at the lowest cost. A quite different approach is to judge a policy based on a "cost-benefit" framework (e.g. Eckaus, 1992) where a decision-maker seeks to minimize the sum of the costs of climate change and the costs of mitigating emissions. This is typically a more flexible approach for assessing a multitude of different policy options since it does not require the environmental goal to be the same across the policies (e.g. Freeman, 2003; Kolstad, 2000; USEPA, 2000). This in turn, requires placing different climate impacts on a common (usually monetary) scale. A metric that is designed to inform a cost-benefit framework may be different to one designed to support a costeffectiveness analysis.

A metric allows emissions to be put on a common scale. In the context of climate change, the common scale is often called " $\mathrm{CO}_{2}$ equivalent" emissions, ${ }^{6}$ which is obtained by multiplying the amount of a given species that is emitted by its metric value. An overall goal of a (cost-effective) climate policy could be to limit the total " $\mathrm{CO}_{2}$-equivalent" emissions so that a given long-term target of the climate policy is met. This could be either through a "target and timetable" type agreement (such as the Kyoto Protocol where the maximum permitted emissions at some given time in the future are specified), or for a type of agreement that is based on, for example, technology standards (such as legislation on emission standards from cars, aviation, etc.). As discussed by several authors (Aaheim et al., 2006; Bradford, 2001; Johansson et al., 2006; Manne and Richels, 2001; Shine et al., 2007), for a cost-effective policy which sets a long-term limit on the allowed change in global temperatures (or any other climate parameter), the relative importance of different emissions will depend on whether the time at which that limit is likely to be reached is far in the future or not. This information is known in advance and can be communicated to decisionmakers, so that it is taken into account when there are investments in new long-lasting equipment (e.g. new power-plants that will be in operation for 40-50 years).

The Kyoto Protocol includes a provision for emission trading amongst Annex 1 (i.e. developed world) countries with the aim of promoting cost-effectiveness, although details of any such emission trading schemes are left for bilateral or multilateral agreement amongst the signatories of the Protocol. If those agreements are multi-gas approaches, then metrics are required to support them.

\footnotetext{
${ }^{6}$ Note that there are two different usages of $\mathrm{CO}_{2}$ equivalence which serve distinct purposes. The total radiative forcing from many different sources is sometimes presented as an equivalent concentration of $\mathrm{CO}_{2}$ in the atmosphere that would be required to give the same forcing. In the usage here, it is the emissions that are placed on a $\mathrm{CO}_{2}$-equivalent scale, and the equivalence depends sensitively on the choice of metric, and parameters within that metric.
} 
Of course, emissions trading is not the only route to facilitate costeffectiveness; a range of possible policy measures relevant to the transport sector is discussed in Kahn Ribeiro et al. (2007).

Ultimately, climate change policymaking requires prioritizing among different groups in the society (in both space and time) and among different impacts of emissions. Many of the transport activities that affect climate are also associated with other environmental concerns, such as air quality, noise and stratospheric ozone. There is the risk that measures aimed at ameliorating one environmental problem will exacerbate another. One example of this would be if a chemical species that cools the climate but, at the same time causes air pollution (e.g. $\mathrm{SO}_{2}$, or organic carbon particles), were given negative climate metric values that encourage increased emissions. Attempts to develop metrics that consider more than one environmental issue are underway (and this is, for example, the motivation behind the analysis presented by Marais et al., 2008). In the absence of such common metrics for a range of environmental issues, it is possible that policymakers will combine, in some sub-optimal way, different metrics that address each environmental issue independently.

\subsection{Factors influencing metric choices}

There are several issues behind the choice of metrics for comparing the climate effects of different emissions. We will discuss these to motivate the particular choices adopted here. The discussion will concentrate on climate metrics, as these are the subject of most current debate although, in general, the arguments are applicable to ozone depletion metrics. A comprehensive review of the application of climate metrics can be found in Fuglestvedt et al. (2003).

The first end-point on Fig. 1 that allows any direct comparability of the climate impacts is radiative forcing, and this end-point is used by the GWP. Of arguably greater relevance is the surface temperature change and metrics which include temperature have been proposed - see below. Beyond temperature change, other end-points are more difficult to quantify, given current understanding; they are likely to be more contentious amongst physical and economic scientists, as they require additional assumptions concerning, for example, the poorly-known relationship between temperature change and damage. Nevertheless, metrics which are further down the cause-effect chain may be more useful to policymakers; in the application of, say, the GWP, implicit assumptions about the relationship between $\mathrm{CO}_{2}$-equivalent emissions and the wider impact on society have to be made by them in evaluating a policy. In a policymaking context, it may be preferable to tolerate increased scientific uncertainty, if the relevance of the environmental effect is clearly higher (USEPA, 2000; Schmalensee, 1993).

Once an end-point is chosen, a number of additional decisions have to be made in the production of a metric; many of these decisions go beyond purely physical science considerations and involve value judgements as regards the important parameters and timescales. Questions that need to be considered include:

\subsubsection{What kinds of emissions are considered?}

These could be, for example, a pulse emission, which would compare the future impact of emissions in a given year. They could consider the impact of emission changes sustained for some period in the future; either over the time horizon of the integration or for a limited period; e.g. 30 years (Berntsen et al., 2005; Smith, 2003). Or they could consider some particular future scenario of emissions. We adopt the pulse approach here, partly on the grounds that the choice of sustained emission metrics implies a commitment for future policymakers, and partly because pulse emissions possess a greater generality; they can be combined to produce metrics for the sustained case or any emissions scenario case, as will be illustrated in Section 5.4 (at least to the extent that the metric values do not change as the background state changes). All choices of types of emission perturbations are somewhat artificial in construct and different choices serve different purposes.

\subsubsection{How are the time-dependence of the impacts taken into account?}

It could be the impact at some given time in the future, or the impact integrated over some given time horizon. It could be the absolute value of a parameter, or its rate of change. Once this is chosen, it is necessary to choose a value of the time horizon, and decide whether some form of discounting is applied; discounting (e.g. where the impact is weighted by a factor of the form $\exp (-r t)$, where $r$ is a discounting rate and $t$ is time) is often employed within economics, so that scarce resources can be allocated most appropriately across time or future generations when choosing among different options. ${ }^{7}$ The application of discounting and the choice of the form and strength of the discounting are controversial when applied to climate science (see e.g. Sherwood, 2007; Nordhaus, 2007; Stern, 2007 and references therein). However, the judgements that are required in choosing whether and how to apply discounting are not distinct from the choice of time horizon in the GWP; the choice of time horizon can be interpreted in terms of a discounting rate (see Fuglestvedt et al., 2003), although the effective discount rate for a given time horizon varies with the lifetime of the emitted species. Arguably, the way that discount rates are effectively embedded within GWPs is an undesirable property of a metric, as the user is unable to vary the assumptions in a transparent manner. It may be more desirable to extract any value-laden judgements from a metric, so that they may be applied explicitly by the user (Nordhaus, 2007; Weitzman, 2007). Choices concerning time horizon and discounting are particularly important as they strongly affect the perception of whether it is more effective to mitigate short-lived or long-lived gases.

\subsubsection{What level of spatial disaggregation is considered?}

There are two distinct issues. First, even the global-mean value of an emission metric may depend significantly on where the emission takes place (both geographically and in terms of altitude). There has been significant focus on this for $\mathrm{NO}_{x}$ (Berntsen et al., 2005; Fuglestvedt et al., 1999; Wild et al., 2001; Derwent et al., 2008) - see Section 7 where the radiative forcing resulting from a given emission of $\mathrm{NO}_{x}$ varies by over an order of magnitude depending on where it is emitted. In general, ozone is more readily created from $\mathrm{NO}_{x}$ emitted at low latitudes and in the upper troposphere. Hence, particularly for aviation and shipping, which can cover large distances, the climate impact of emissions can vary greatly along the route. The situation is perhaps most acute when considering contrail formation from aviation; the meteorological conditions necessary for the formation of persistent contrails depend sensitively on atmospheric conditions which can change rapidly both in time and in the horizontal and vertical directions; and the sign of the radiative effect of the contrails depends on the

\footnotetext{
7 Discounting has three components (see e.g. Weitzman, 2007). There is the component related to the pure rate of time preference $(\delta)$. This is an "impatience factor" that describes preference of having something today versus at some time in the future. There is also a component which is the product of growth rate of consumption $(g)$ and elasticity of marginal utility $(\eta)$. The latter describes how the extra utility of one additional unit of a good depends on the level of utility and goods already achieved. The discount rate $r$ is then given as $r=\delta+\eta g$ (Weitzman, 2007). The curvature of the utility function is a major source of uncertainty for what constitutes a long-term discount rate. The parameters $\delta$ and $\eta$ are tightly coupled to value considerations.
} 
time of day at which they form (Myhre and Stordal, 2001; Stuber et al., 2006). Contrail formation also depends to some extent on the overall propulsion efficiency of the aircraft (e.g. Schumann, 2005). In principle, the climate effect of, and hence emission metrics for, aviation ought to be assessed on a flight-by-flight basis, and would depend on the different altitudes at which the aircraft flies and the prevailing meteorological conditions and the variation of daylight along the route. This is unlikely to be possible for the foreseeable future, and so some kind of fleet-averaged effect is one pragmatic solution.

The second distinct issue is that a globally-averaged radiative forcing or temperature change does not give any information on the geographical distribution of the impact; even a spatially homogeneous radiative forcing would lead to distinct geographical patterns of temperature response, as the response pattern is strongly affected by feedbacks within the climate system (e.g. Boer and Yu, 2003). And even if the temperature response was homogeneous, this would not imply that the impact (on, for example ecosystems, agriculture or economy) to that response is homogeneous. A particular example of relevance to the climate impact of the transport sector concerns the response to $\mathrm{NO}_{x}$ emissions. As will be discussed in Section 6, $\mathrm{NO}_{x}$ emissions lead to an increase in ozone and a decrease in methane, which result in radiative forcings which are, to first order, of around the same size but opposite in sign. However, while they oppose each other in the global mean, they do not necessarily do so locally. Fig. 2 shows an idealized case of surface temperature changes due to a sustained emission of $\mathrm{NO}_{x}$ from Europe, calculated using a chemical transport model and a climate model. In this case, the increase in ozone is mostly restricted to the northern hemisphere, while the longer-lived methane change is global in extent. The simulations indicate that broadly one hemisphere warms while the other cools, and the global-mean temperature change may not be a reliable guide to the total impact. Discussions on whether, or how, such a response can be addressed in a policy are in their infancy and are discussed briefly in Section 6. In summary, there are two distinct aspects that lead to a regional variation in the climate response to a forcing one is the geographical variation in the response of the climate system even for a perfectly homogeneous radiative forcing, and the other is the geographical distribution of the forcing; for short-lived components, climate models indicate that climate response is predominantly in the hemisphere in which they are emitted.

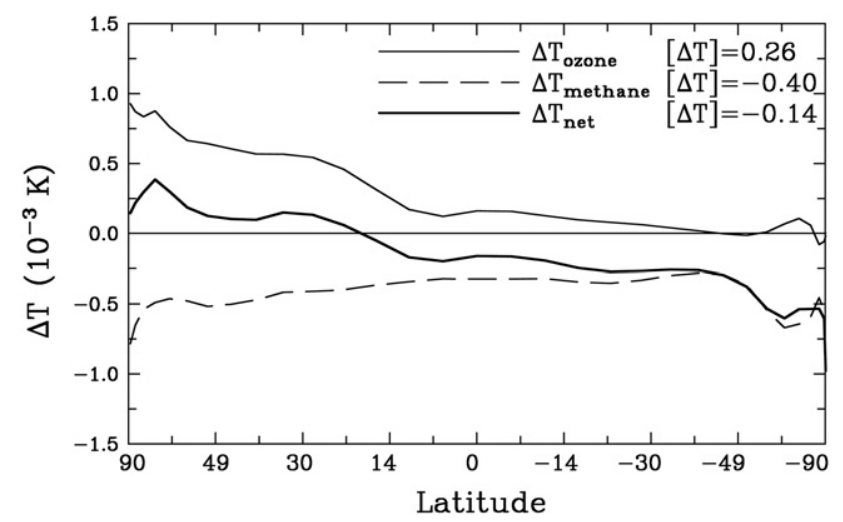

Fig. 2. Surface temperature changes from GCM calculations where an idealized emission of $\mathrm{NO}_{x}$ from the surface in Europe is traced through its impacts on ozone, methane, radiative forcing and temperature change. The surface temperature changes are shown for ozone changes only (thin solid line), methane changes only (dashed line) and the net effect (thick solid line). It shows that the strong global-mean cancellation between the two impacts (see [] values in the legend) are made up of a northern hemisphere warming, where the ozone impact dominates over methane, and a southern hemisphere cooling where methane dominates over ozone (from Shine et al., 2005a).
It is important to distinguish between the fact that equal-mass emissions from different regions can vary in their global-mean climate response and that the climate response to emissions can also have a regional component irrespective of the regional variation in emissions. These two aspects of "regionality" are quite distinct in their implications for metric development.

It seems likely that further progress in understanding the relationship between climate change and damage (whether it is to ecosystems or to the economy) will be necessary to enable improved analysis of cases where global-mean compensation in climate change is not matched at the regional or hemispheric level. Dealing with this sort of compensation is not new in the context of economics or even within the context of environmental impacts. However, it is particularly challenging for the case of climate damages because of the inherent complexity of defining this damage and the very long timescales over which impacts of even current activities are anticipated to occur. Therefore, there is less empirical evidence presently available to assess some of the important trade-offs (as compared, for example, to data that are available to statistically analyze hospital reports of health incidences and their relationship to local air quality - a common practice for understanding concentration-response relationships for different surface pollution impacts).

\section{Possible climate metrics}

\subsection{General considerations}

The above discussion indicates that there are many unresolved issues in metric design and, to serve current needs, a pragmatic approach is needed. In Section 7 we present values for two different climate metrics, the GWP, and a more recently proposed metric, the Global Temperature Change Potential (GTP). For the most part, the emphasis will be on metrics that compare the climate impact of emissions, but it is important to discuss the role of radiative forcing, both as a metric in its own right, but also to distinguish it clearly from emission metrics.

The GWP has been adopted by the Kyoto Protocol to implement a multi-gas approach and is widely used in policymaking. Scientific updates of GWPs are regularly published by the IPCC, although these are not used in the reporting of greenhouse-gas emissions to the UNFCCC for the Kyoto Protocol, which adopts those presented in IPCC's Second Assessment Report (IPCC, 1996). There is no clear definition of what GWP is an indicator of - i.e. which aspects of climate change it is a proxy for. Several studies have evaluated the equivalence of GWP-weighted emissions and emphasized that GWP-equivalence does not imply any equivalent temperature response except in certain idealized situations. Thus, the use of GWPs has been strongly debated in the scientific literature (e.g. Rotmans and Den Elzen, 1992; Fuglestvedt et al., 2003; O'Neill, 2000; Skodvin and Fuglestvedt, 1997; Smith and Wigley, 2000) with strong criticism especially from economics community (Bradford, 2001; Eckaus, 1992; Manne and Richels, 2001; Tanaka et al., in press). However, no alternative has so far become widely accepted. A brief summary of the merits of the GWP versus other metrics is given in IPCC (2009).

The IPCC has refrained from presenting its own calculations of GWPs for short-lived species, but has chosen instead to review and present values available in the literature. The main reasons for this are the complexities arising from the chemical/physical indirect effects and the spatial and temporal variations. There are some publications with GWPs and alternative metrics for short-lived species (e.g. Berntsen et al., 2005; Bond and Sun, 2005; Boucher and Reddy, 2008; Collins et al., 2002; Derwent et al., 2008; Shine et al., 2007; Stevenson et al., 2004; Wild et al., 2001). For the 
short-lived, chemically-active climate gases there are (at least) two dimensions to the difficulties related to establishing metrics (Shine et al., 2005a): i) design of the metric and ii) inter-model differences. Using $\mathrm{NO}_{x}$ as an example, the response depends significantly on where the emissions take place, and the global-mean response is heavily influenced by the compensation between the individual positive $\left(\mathrm{O}_{3}\right)$ and negative $\left(\mathrm{CH}_{4}\right)$ responses to the emissions. In this case, the GWP may not be an adequate measure of climate impact. Furthermore, the status of knowledge and ability to model the chemical and climate response to $\mathrm{NO}_{x}$ emissions is relatively low and there are large variations between the results from various models; however some of this variation may reflect differences in experimental design, rather than differences in the underlying representation of atmospheric processes.

The adoption of the GWP here may be considered controversial in the context of transport emissions, given the discussions in some of the prior literature. For example, IPCC (1999) stated that the GWP "has flaws that make its use questionable for aviation emissions" and that "there is a basic impossibility of defining a GWP for aircraft $\mathrm{NO}_{x}$ ". Wit et al. (2005) echo these sentiments, concluding that "GWPs are not a useful tool for calculating the complete suite of aircraft effects". An undesirable side effect of the negative stance of IPCC (1999) is that it has led some policymakers and other groups to apply the Radiative Forcing Index (RFI) (see Section 5.2 and Appendix 1) as if it is some kind of alternative to the GWP (see the discussions on the problems in applying the RFI in Forster et al., 2006, 2007b; Wit et al., 2005).

Others have taken a more pragmatic stance, and attempted to develop GWPs for aviation emissions, whilst recognising the caveats. Johnson et al. (1992) were perhaps the first to present GWPs specifically for aviation $\mathrm{NO}_{x}$. More recently, Wild et al. (2001) and Stevenson et al. (2004) have generated GWP values (although they did not label them as such) using 3-D CTMs. Forster et al. (2006, 2007b) have generated GWP values for a range of aviation emissions. Marais et al. (2008) have considered a range of metrics (RF, integrated temperature change, and integrated damage), and the impact of uncertainties, in their evaluation of aviation emissions, and included estimates of the economic impact of these emissions. There have also been attempts at deriving GWPs that specifically address the issue of the impact of changes in flight altitude. The first appears to be by Klug and colleagues in a series of unpublished reports as part of the EC Framework 5 Cryoplane project. More recently, Svensson et al. (2004) have provided GWP values for aviation, based partly on the Klug approach.

However, there are arguments in favour of using GWPs. One is in terms of continuity with their application in other areas of climate policy, where GWPs are an accepted metric; there would undoubtedly be a cost associated with adopting and implementing new metrics, although it must be recognised that there might also be a cost to using GWPs if they led to faulty decisions (Aaheim et al., 2006; Godal and Fuglestvedt, 2002; Johansson et al., 2006; O’Neill, 2003). A second, is that many of the difficulties in defining values for the GWP (for example, dependence on flight altitude and conditions) are common to all methods of assessing the climate impact of aviation and transport in general, and indeed GWPs can act as one tool for illustrating the extent of that uncertainty (see Section 7). Third, and as noted above, the failure to provide values for the GWP may lead to even less suitable metrics being used. It is recognised here that, unlike the Kyoto gases, it is certainly generally not possible to prescribe a single value of the GWP for short-lived emissions, which are independent of location and conditions at the time of emission.

It is also useful to consider possible alternatives to the GWP, even if they do not have the same level of maturity and acceptance.
In the context of policy which has specific targets in terms of temperature change at some point in the future, Manne and Richels (2001) proposed a measure that takes into account the fact that the impact of emissions of short-lived species depends sensitively on how close the target is. They used an integrated-assessment model which included a reduced-form description of the energy sector and economics, in addition to the physical components of the climate system. A metric which has a similar behaviour but has a purely physical science framework is the GTP (Shine et al., 2007) which has also been used for example by Boucher and Reddy (2008) and has attracted wider interest (IPCC, 2009). One difference however is that Manne and Richels (2001) account for the effects of emissions after the time at which stabilization occurs, although their model's behaviour in this regime may be dictated more by assumptions about abatement costs than by physical aspects of the climate system. An attraction of the GTP is that it requires essentially the same inputs as the GWP but represents the response of the global-mean surface temperature. Hence it provides a different perspective on the relative importance of emissions of different species and how this changes over time. Additionally, because it considers temperature change, the GTP is further down the cause and effect chain from emissions to impacts and may, therefore, be a preferable metric even though, as noted in Section 2, it is, therefore, subject to a greater uncertainty. Tol et al. (2008) show that under certain assumptions the GWP may be viewed as appropriate for a cost-benefit framework, whereas the GTP may be viewed as appropriate for a cost-effective framework (see Section 4.1).

\subsection{Radiative forcing}

Radiative forcing is a standard way of comparing the effects of the various emissions on climate. It is commonly presented as the present-day $\Delta F$ relative to pre-industrial times (e.g. IPCC, 1995, 1996, 2001, 2007), but it can be used to compare the effect of changes between any two points in time. The strengths and weaknesses of the concept of radiative forcing have been discussed in detail in these IPCC reports. We do not use it here as a metric, as our emphasis is on emission metrics that compare the climate impact of the emission of one substance compared to the emission of some reference gas. Nevertheless, $\Delta F$ is an important input to these emission metrics and so further discussion is merited.

One application of radiative forcing has been to present the total radiative forcing of a given sector (and, in particular, for aviation) as that sector's total $\Delta F$ divided by the $\Delta F$ due to its $\mathrm{CO}_{2}$ emissions this yields the so-called Radiative Forcing Index (RFI); in itself, the $\mathrm{RFI}$ is a useful concept for indicating the contribution of non- $\mathrm{CO}_{2}$ emissions at a given time. Unfortunately, the RFI has been misapplied in some quarters as if it were an emission metric, which it clearly is not, as it is dependent on the history of past emissions. A discussion of some of the problems in applying the RFI as an emissions metric is given in Appendix 1 but, briefly, the application of the RFI appears inconsistent with the use of GWPs within the Kyoto Protocol, its suggested use seems to have been restricted to a single sector (i.e. aviation) and its use could result in inappropriate measures being taken, in attempts to reduce the climate impact of emissions from a sector.

The $\Delta F$ at a given time is a "backward looking" measure. In the case of the $\Delta F$ relative to pre-industrial times, it quantifies the radiative effect due to the accumulated change in abundance as a result of all emissions during that period. For long-lived gases, the $\Delta F$ may be due to emissions occurring over the preceding decades/ centuries. For short-lived species, it may only be emissions over the previous weeks that have contributed. This view is useful for attribution studies and for understanding the anthropogenic effects 
on climate. For policymaking, a future perspective is more relevant and then $\Delta F$ can be used as an indicator of climate response to a) current emissions or b) emission scenarios. Alternative a) isolates and then quantifies the effect of a known emission in a given year, while alternative b) introduces the effects of several factors required for any scenario analysis or forecast, such as assumptions about projected technological and economic development and population growth.

It is the time variation of $\Delta F$ (which is determined by a combination of the lifetime of the species contributing to the forcing and their emission history) which ultimately determines the effect on current (and future) temperatures. One should also keep in mind the very different behaviour the agents show after the chosen year due to the very different lifetimes; in other words, this picture does not say anything about the future role of the various $\Delta F$ agents.

Fig. 3a shows the $\Delta F$ history induced by emissions from the transport sector up to 2000 (using emission numbers from QUANTIFY $^{8}$ ) with zero transport emissions after this time. The standard $\Delta F$ diagram referred to above just shows the instantaneous values for (typically) year 2000 relative to pre-industrial times. Fig. 3a also shows that the perturbation of $\mathrm{CO}_{2}$ is very longlived while $\mathrm{O}_{3}$, black carbon $(\mathrm{BC})$ and sulphate $\left(\mathrm{SO}_{4}\right)$ die out quickly after the emissions stop. Fig. $3 \mathrm{~b}$ shows the temperature response for these emissions. A delay of about a decade can be seen which is due to the thermal inertia of the ocean.

One possible extension to the radiative forcing is to take into account the so-called efficacy of different climate forcings (e.g. Forster et al., 2007a; Hansen et al., 2005 and, specifically in the context of aviation, Ponater et al., 2006). The efficacy measures the ratio of $\lambda$ (see Section 3 ) for a given mechanism, to the value of $\lambda$ for a $\mathrm{CO}_{2}$ doubling. An important underlying assumption in the early application of radiative forcing was that the same forcing from different climate change mechanisms, would lead to the same climate response, so that the efficacy was always unity. It is now recognised from climate model experiments, that the climate response from, say $1 \mathrm{Wm}^{-2}$ of forcing due to a change in $\mathrm{CO}_{2}$, may differ from the response to the same forcing due to, for example, black carbon aerosols. The reasons for the departure of efficacy from unity are not always easy to diagnose (see discussion in Forster et al., 2007a and references therein), but include differences in the geographical distribution of forcings (for example, GCM calculations indicate high-latitude forcings are generally more effective than low latitude forcings), differences in the vertical distribution of the forcing (which can impact on cloud, temperature and stratospheric water vapour response) and differences in the degree of dominance of short and longwave radiation in driving the forcing (which affects the surface/atmosphere partitioning of the forcing).

Conceptually it is quite straightforward to incorporate efficacy in metrics, as it just involves multiplying any forcings by the efficacy. However there is as yet insufficient consensus amongst models to confidently assign values for the efficacy even for quite straightforward forcings, such as that resulting from a change in incoming solar irradiance at the top of the atmosphere (Forster et al., 2007a). In the calculations presented here, the default assumption will be that the efficacy has a value of 1 although, as discussed in Forster et al. (2007a), individual model studies have found values for various forcings can lie in the range from about 0.6 to 1.7. Specifically in the context of aviation, Ponater et al. (2006) found in their particular GCM that efficacy varies from 0.59 for contrail forcing to 1.37 for ozone forcing. We are unaware of any

\footnotetext{
${ }^{8}$ QUANTIFY is an EU FP6 Integrated Project Quantifying the Climate Impact of Global and European Transport Systems - see www.ip-quantify.eu.
}
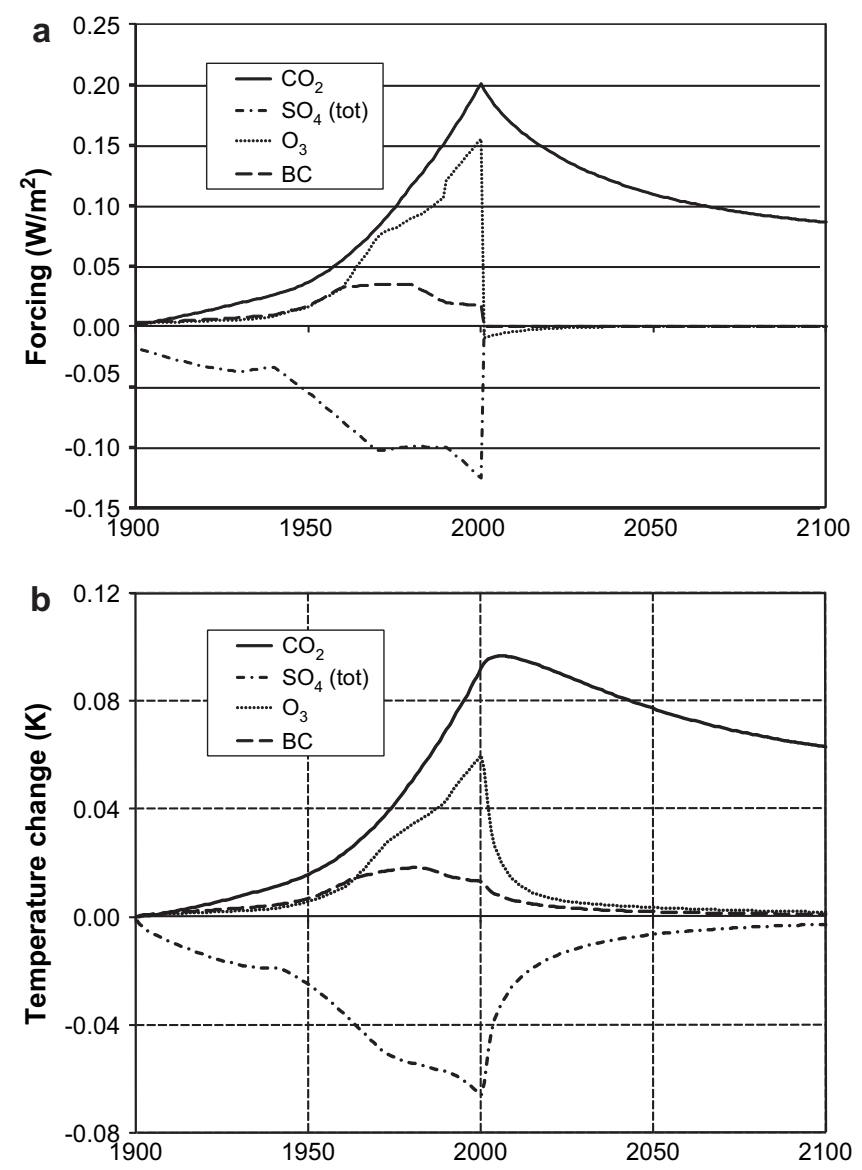

Fig. 3. a) Forcing history due to emissions of $\mathrm{CO}_{2}, \mathrm{SO}_{2}, \mathrm{BC}$ and ozone pre-cursors from the transport sector up to 2000 with zero emissions after this time. b) Temperature response for the same emission histories. The CICERO SCM has been used for these calculations with emission numbers from QUANTIFY. ( $\mathrm{SO}_{2}$ effects include direct and first indirect effect.)

published studies that have examined the efficacy specifically for the other transport sectors.

A further argument for using an efficacy of 1, given current understanding, is that departures from this value are, at least partially, dependent on the spatial pattern of forcing; as an example, for ozone forcing due to $\mathrm{NO}_{x}$ emissions from the surface, climate models indicate that the efficacy for emissions fom Europe may differ noticeably from that derived for emissions from southeast Asia (Berntsen et al., 2005). Application of the efficacy should, ideally, then be dependent on both the climate change mechanism, and the location of the emissions; to maintain transparency, it would seem undesirable to impose a blanket efficacy for a given forcing irrespective of where the emission occurs. Clearly a better understanding of impact of transport emissions on climate requires an improved understanding of the efficacy.

\subsection{Global Warming Potential (GWP)}

The Global Warming Potential (GWP) is based on the timeintegrated radiative forcing due to a pulse emission of a unit mass of gas. It can be quoted as an absolute GWP (AGWP) (e.g. in units of $\mathrm{Wm}^{-2} \mathrm{~kg}^{-1}$ year) or as a dimensionless value by dividing the AGWP by the AGWP of a reference gas, normally $\mathrm{CO}_{2}$. A user choice is the time horizon over which the integration is performed. The Kyoto Protocol has adopted GWPs for a time horizon of 100 years. The choice of time horizon in the Protocol is, to our knowledge, not 
based on any published conclusive discussion and IPCC science assessments have generally presented GWPs for three time horizons, 20, 100 and 500 years. The use of different time horizons, would reflect different value judgements related to the importance of impacts that may occur in the far future - see Section 4 .

For a gas $x$, if $A_{x}$ is the specific radiative forcing (i.e. the radiative forcing per $\mathrm{kg}$ ), $\alpha_{x}$ is the lifetime (and assuming its removal from the atmosphere can be represented by exponential decay), and $H$ is the time horizon then

$$
\operatorname{AGWP}^{x}(H)=\int_{0}^{H} A_{x} \exp \left(-t / \alpha_{x}\right) \mathrm{d} t=A_{x} \alpha_{x}\left[1-\exp \left(-H / \alpha_{x}\right)\right]
$$

The AGWP for $\mathrm{CO}_{2}$ is more complicated, because its atmospheric response time (or lifetime of a perturbation) cannot be represented by a simple exponential decay. The AGWP for $\mathrm{CO}_{2}$ used here is discussed in Section 7. The AGWP concept can easily be extended to include the efficacy of different forcings (Fuglestvedt et al., 2003), see Section 5.2. Note that the parameters used in calculating the AGWP may be dependent on the choice of background state, but it is convention to use present-day conditions.

In the early discussions of the GWP (Fisher et al., 1990b) an infinite time horizon was chosen. In these circumstances, the GWP has an alternative, and perhaps more physical, interpretation, as the ratio of the equilibrium warming due to a sustained emission of a gas, compared to that due to the reference gas (e.g. Shine et al., 2005b) (at least when the efficacy for both gases is unity). This is of importance here as, when an infinite time horizon is adopted, the GWP is essentially the direct analogue of the Ozone Depletion Potential which will be discussed in Section $8 .^{9}$

\subsection{Global Temperature Change Potential (GTP)}

A more recently proposed group of metrics (Shine et al., 2005b) are the pulse and sustained Global Temperature Change Potential (GTP) which have rather different characteristics to the GWP (they are "end-point" metrics - i.e. the temperature change at a particular time in the future, rather than being integrated over time ${ }^{10}$ ). Here, only the pulse form of the GTP is discussed because of its potential relevance to target-orientated climate policy; as noted in Section 5.3, the sustained GTP and the pulse GWP are quite closely related. An application of the pulse GTP for particular scenarios is presented later in this section. The generic definition of the absolute GTP (AGTP) can be given by

$$
\operatorname{AGTP}(H)=\int_{0}^{H} \Delta F(t) R(H-t) \mathrm{d} t
$$

\footnotetext{
${ }^{9}$ Note that this interpretation of the GWP as the impact of a sustained emissions on temperature, should not be confused with the sustained Global Warming Potential (see Berntsen et al., 2005 and references therein) where the time-integrated radiative forcing due to a sustained emission is computed - the use of this metric is not pursued here.

${ }^{10}$ Note that it would be possible to use radiative forcing itself as an end-point, rather than temperature; we are unaware of any attempts to explore the use of such a metric. Certainly for short-lived species and relatively long-time horizons it would act to make them appear less important than the GTP, which retains a memory of the perturbation to the climate system. Nevertheless, it would seem appropriate for a target cast in terms of radiative forcing. Likewise, it would be possible to cast the GTP in a time-integrated form, which may be appropriate given certain assumptions about the dependence of damage on climate change.
}

where $R(H-t)$ gives the surface temperature response at time $H$ due to a radiative forcing at time $t$.

To allow a transparent formulation of the GTP, Shine et al. (2005b) adopted a very simplified climate model (Equation (4)) which allowed an analytical form of the GTP to be derived, although this is by no means a requirement. The inclusion of this climate model means that additional parameters are required to be defined - the timescale of the climate response, $\tau$, and the heat capacity of the climate system, $C$ (or equivalently, $C$ and the climate sensitivity parameter, $\lambda$ - the three parameters are related since $\tau=C \lambda$ - see Section 2).

For this simple model, the absolute GTP (or AGTP) for gas $x$ is given by

$$
\operatorname{AGTP}(H)=\frac{1}{C} \int_{0}^{H} \Delta F(t) \exp \left(\frac{t-H}{\lambda C}\right) \mathrm{d} t .
$$

Assuming, as with the AGWP, a pulse emission, with the radiative forcing decaying exponentially with time then

$$
\operatorname{AGTP}^{x}(H)=\frac{A_{x}}{C\left(\tau^{-1}-\alpha_{x}^{-1}\right)}\left[\exp \left(-H / \alpha_{x}\right)-\exp (-H / \tau)\right]
$$

Again, a more complex relationship is required for $\mathrm{CO}_{2}$ and an alternative expression to Equation (8) is required if $\tau$ and $\alpha$ are equal. The efficacy of climate forcings (Section 5.2) can also be easily incorporated in the concept of the GTP.

Although the simple model given in Equation (8) scores highly for simplicity, here we adopt a somewhat more sophisticated model, that includes a representation of the deep ocean - this has the impact of increasing the climate system's long-term memory to a pulse perturbation in forcing. The impact of different model choices is discussed briefly in Shine et al. (2007, 2005b) who show that neglect of this long-term memory leads to a larger error in the GTP for short-lived emissions at long-time horizons. Since many of the transport-related emissions are indeed short-lived, we adopt the approach of Boucher and Reddy (2008) who used a climate response function derived from a GCM in their GTP analysis. The analytical equations used for calculating the GTP using this approach are presented in Appendix 2. The adoption of this more complex approach has the disadvantage that it is no longer straightforward to alter the climate sensitivity as it is no longer an explicit parameter in the formulation. (See also Berntsen and Fuglestvedt, 2008, for a description of an analytical 2-box model that has been used for analyses of temperature responses to emissions of short- and long-lived components.)

The GTP is generally presented as a ratio of the AGTP for a given species to that of $\mathrm{CO}_{2}$; this means that $\lambda$ appears in both the numerator and denominator of the GTP expression and the GTP is less sensitive to variations in $\lambda$ than the AGTP. However, over the range of uncertainty of $\lambda$, the GTP is still sensitive to the value of $\lambda$ for short-lived species (Shine et al., 2003). Using the Berntsen and Fuglestvedt (2008) 2-box model across the range of "likely" climate sensitivities discussed in Section 2, the black carbon GTP(50) was found to vary by a factor of 2 , the methane GTP(50) varied by about $50 \%$, while for the long-lived gas $\mathrm{N}_{2} \mathrm{O}$ there was essentially no dependence. This is an example of the point noted earlier, in relation to Fig. 1, that increasing relevance of the end-point of a metric is often associated with increasing uncertainty and less accuracy in its quantification.

We choose to concentrate on pulse emissions (rather than applying the concept to scenario of emissions - for example, considering emissions over the lifetime of a car, ship or aircraft) as the pulse emissions can be readily applied to the analysis of policies that implies time-varying emissions. To illustrate the methodology, 
we analyze two policies or technological mitigation options ( $\mathrm{P}_{1}$ and $\mathrm{P}_{2}$ ) with different emissions ( $\mathrm{E}_{1, i}$ and $\mathrm{E}_{2, i}$ respectively) of components $i$ during the time between $t=0$ and a time $t_{\mathrm{e}}$. The impact of the policy is assessed by the (relative) change in temperature at time $t_{\mathrm{t}}$,which is the target (or evaluation) year. (We use Equation (8) for this illustrative example, for simplicity.)

The impacts evaluated by metrics $M$ of the two policies/options are then given by

$I_{1}\left(t_{\mathrm{e}}, t_{\mathrm{t}}\right)=\sum_{i} \int_{0}^{t_{\mathrm{e}}} \mathrm{E}_{1, i}(t) M_{i}\left(t_{\mathrm{t}}-t\right) \mathrm{d} t$

and

$I_{2}\left(t_{\mathrm{e}}, t_{\mathrm{t}}\right)=\sum_{i} \int_{0}^{t_{\mathrm{e}}} \mathrm{E}_{2, i}(t) M_{i}\left(t_{\mathrm{t}}-t\right) \mathrm{d} t$.

For simplicity we compare two idealized policies changing $\mathrm{CO}_{2}$ and methane emissions only, and use the pulse GTP metric with a target time (or evaluation year) 100 years after the emissions start changing. Note that the time horizon used in the GTP for calculating $I_{1}$ and $I_{2}$ is reduced as one approaches the target time. We assume policies $\mathrm{P}_{1}$ and $\mathrm{P}_{2}$ with emissions of $\mathrm{CO}_{2}$ of 10 and 5 units, and emissions of methane of 1 and 2 units respectively. Fig. 4 shows the impacts normalized to the impact of $P_{1}$ after 100 years $\left(I_{1}(100,100)\right)$ as function of duration of emission reductions $\left(t_{\mathrm{e}}\right)$. With these (arbitrary) assumptions, the figure shows that if the emission changes last for less than about 65 years then adopting $\mathrm{P}_{2}$ would contribute less to warming at the target time ( $t_{\mathrm{t}}=100$ years) than $\mathrm{P}_{1}$. If the policies last longer than that, $\mathrm{P}_{1}$ with less methane with its shorter lifetime and larger specific radiative forcing, will cause smaller warming at $t_{\mathrm{t}}$.

In this assessment we have assumed a constant background atmosphere, in common with assumptions underlying the calculation of the GWP by IPCC. Changes in the background atmosphere can impact on aspects such as the lifetimes of gases and their specific radiative forcings (see review by Fuglestvedt et al., 2003 and references therein), their incorporation requires assumptions about the future evolution of the state; in some cases that have

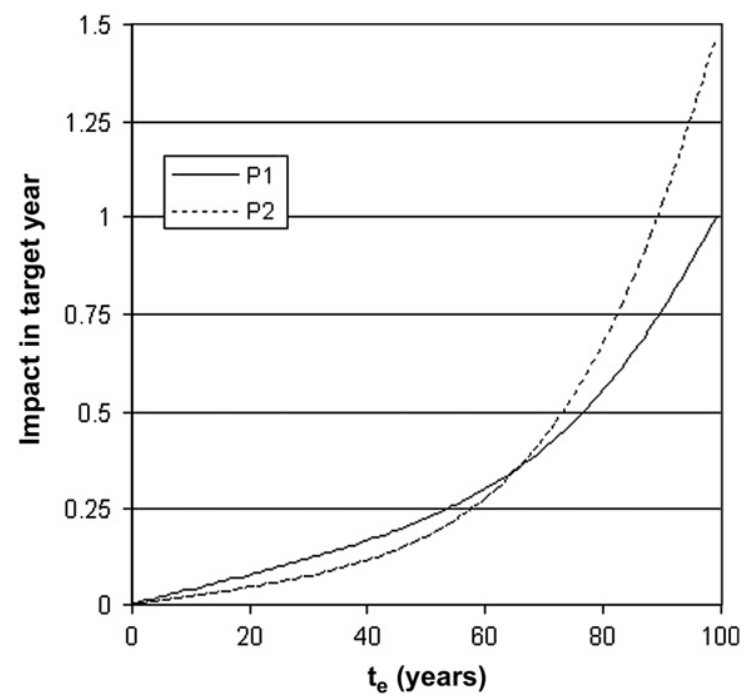

Fig. 4. Illustration of the use of a pulse-based GTP for scenario studies. Normalized climate impacts of policies $\mathrm{P}_{1}$ and $\mathrm{P}_{2}\left(\mathrm{P}_{1}: 10\right.$ and 1 units of $\mathrm{CO}_{2}$ and $\mathrm{CH}_{4}$ emissions per year respectively and $\mathrm{P}_{2}: 5$ and 2 units of $\mathrm{CO}_{2}$ and $\mathrm{CH}_{4}$ emissions per year respectively). The impacts are evaluated using the GTP with target time at 100 years after $t=0 . t_{\mathrm{e}}$ is the duration of emission reductions. See text for discussion. been considered, notably for $\mathrm{CO}_{2}$, the effect of changes in perturbation lifetime and specific forcing appear to partly compensate each other (Caldeira and Kasting, 1993).

\subsection{Summary}

Table 1 summarises and compares the role of radiative forcing and the emission metrics, their usage, advantages and disadvantage. It assumes that the furthest end-point used in terms of the impact is temperature, although it can clearly be extended to other end-points.

\section{Nature of transport emissions, radiative forcing and climate response}

There are four main mechanisms by which emissions from transport affect climate: (i) by emission of direct greenhouse gases, (ii) by emission of indirect greenhouse gases, i.e. gases that are precursors of tropospheric $\mathrm{O}_{3}$ and/or affect the oxidation capacity of the atmosphere, (iii) by emission of aerosols or aerosol pre-cursors through their direct effect (either while in the atmosphere or by changing the surface albedo, following deposition there), and (iv) by emission of aerosols or aerosol pre-cursors that directly trigger changes in the distribution and properties of clouds. We discuss these briefly here - more details in the context of the transport sectors are given in the other components of this assessment (Eyring et al., 2010; Lee et al., 2010; Uherek et al., 2010). A more extended discussion of the climate role of these emissions, in a more general context, can be found in, for example, Forster et al. (2007a).

Potentially important sources of radiative forcing from the aviation sector, aircraft contrails, and aviation-induced cirrus, do not easily fit into the above four categories. Contrails are triggered by emissions of water vapour from the aircraft engine, but the formation of persistent contrails is highly dependent on the atmospheric state (see Lee et al., 2010). In particular, a necessary condition is that the atmosphere is supersaturated with respect to ice. The radiative forcing is caused by the condensation of the water vapour already in the atmosphere, with the emission by the engine acting as the trigger for this process. In terms of metric development, this means that there is not as direct a correspondence between an emission and a consequent forcing, as there is with other sources of forcing.

Emissions of $\mathrm{CO}_{2}$ and HFCs lead to a positive $\Delta F$ from increased atmospheric levels of these gases. Emissions of $\mathrm{N}_{2} \mathrm{O}$, CFCs, HCFCs and halons lead to a positive $\Delta F$ from increased atmospheric levels of these gases, but in addition, cause negative $\Delta F$ via reductions in stratospheric $\mathrm{O}_{3}$.

$\mathrm{CH}_{4}$ emissions cause a positive $\Delta F$ by increasing atmospheric $\mathrm{CH}_{4}$ levels, stratospheric $\mathrm{H}_{2} \mathrm{O}$ and tropospheric $\mathrm{O}_{3}$ levels (in the presence of $\mathrm{NO}_{x}$ and solar radiation). The increases in $\mathrm{CH}_{4}$ levels also reduce tropospheric $\mathrm{OH}$ which leads to further increases in the levels of $\mathrm{CH}_{4}$ (a positive feedback loop) and other gases removed by $\mathrm{OH}$ (e.g. $\mathrm{HFCs}$ ). If $\mathrm{CH}_{4}$ emissions are generated by fossil carbon, they will also cause a positive forcing via degradation to $\mathrm{CO}_{2}$ in the atmosphere.

$\mathrm{CO}$ and volatile organic carbon (VOC) emissions cause insignificant direct $\Delta F$. However, they cause positive forcing by decreasing the $\mathrm{OH}$ levels and thereby increasing the $\mathrm{CH}_{4}$ levels and other gases removed by $\mathrm{OH}$. They also cause positive $\Delta F$ via tropospheric $\mathrm{O}_{3}$ formation. The effect on $\mathrm{CH}_{4}$ and $\mathrm{O}_{3}$ depends where the emissions occur. If the emissions are from fossil carbon, they will also cause a positive forcing via degradation to $\mathrm{CO}_{2}$ in the atmosphere. $\mathrm{CO}$ and VOC emissions also cause positive $\Delta F$ via changes in $\mathrm{O}_{3}$ controlled by the $\mathrm{CH}_{4}$ perturbation (we will call this the "methane-induced 
Table 1

Summary of possible global-mean metrics.

\begin{tabular}{|c|c|c|}
\hline Metric & Usage and advantages & Disadvantages \\
\hline$\overline{\text { All }}$ & & $\begin{array}{l}\text { Difficulty in quantifying many effects, given current scientific } \\
\text { understanding Conceptual difficulty in handling the compensation } \\
\text { between opposing forcings on a global level when they do not } \\
\text { compensate locally. }\end{array}$ \\
\hline $\mathrm{RF}$ (present), $\Delta T$ (present) & $\begin{array}{l}\text { Gives impact of all current and past emissions on } \Delta F \text { and } \Delta T \text { at } \\
\text { the present. Relevant for attribution of present climate change. }\end{array}$ & $\begin{array}{l}\text { Temperature metrics require assumption of climate sensitivity } \\
\text { parameter. Both assume that emission inventories are available for all } \\
\text { past emissions of climate relevant species. }\end{array}$ \\
\hline$\Delta F$ (future), $\Delta T$ (future) & $\begin{array}{l}\text { Gives impact of all current and future emissions on } \Delta F \text { and } \Delta T \text { at } \\
\text { some future date. }\end{array}$ & $\begin{array}{l}\text { As above, but with additional uncertainty due to difficulty in defining } \\
\text { future emission scenarios. }\end{array}$ \\
\hline $\begin{array}{l}\Delta F \text { or } \Delta T \text { due to emissions in } \\
\quad \text { one year }\end{array}$ & $\begin{array}{l}\text { Use of } \operatorname{GTP}_{\mathrm{P}}(H) \text { (or similar metric for forcing) gives the impact of } \\
\text { emissions in one year on temperature at some time H years in } \\
\text { the future. }\end{array}$ & $\begin{array}{l}\text { Choice of time horizon has much stronger effect on results than is the } \\
\text { case for GWPs and requires value judgement. }\end{array}$ \\
\hline$\Delta F($ target $), \Delta T($ target $)$ & $\begin{array}{l}\text { Similar to above, but could be used for a policy aiming to restrict } \\
\text { the contribution to } \Delta F \text { or } \Delta T \text { at some chosen or calculated future } \\
\text { target date. It indicates how emissions in a given year contribute } \\
\text { to that target. Shows how relative impact of short-lived } \\
\text { emissions grows as the target time is approached. }\end{array}$ & $\begin{array}{l}\text { As above. Additional difficulties in choosing or calculating the target } \\
\text { date. Some argue that the rate of change of temperature is as important } \\
\text { as the actual change in temperature. }\end{array}$ \\
\hline $\begin{array}{l}\text { Time integrated } \Delta F \text { due } \\
\text { to emissions in one } \\
\text { year - the GWP }\end{array}$ & $\begin{array}{l}\text { This is the method used to characterise the impact of current } \\
\text { emissions within the Kyoto Protocol. Widely used. }\end{array}$ & $\begin{array}{l}\text { Choice of time horizon is essentially a value judgement, conceptually } \\
\text { connected to the application of discounting. }\end{array}$ \\
\hline $\begin{array}{l}\text { Sustained } \operatorname{GWP}(H) \text { and } \\
\qquad \operatorname{GTP}(H)\end{array}$ & $\begin{array}{l}\text { Sustained versions of the pulse GWP and GTP, in which the } \\
\text { effect at time } H \text { is quantified if the current emissions are } \\
\text { sustained between now and } H \text {. }\end{array}$ & Assumes constant future emissions. \\
\hline
\end{tabular}

ozone perturbation", but it is also the sometimes referred to as the "primary-mode" ozone change).

Emissions of hydrogen $\left(\mathrm{H}_{2}\right)$ reduce the levels of $\mathrm{OH}$ and thereby increase the atmospheric levels of $\mathrm{CH}_{4}$ and other gases removed by $\mathrm{OH} . \mathrm{H}_{2}$ also leads to production of ozone in the troposphere. Thus, while $\mathrm{H}_{2}$ has no significant direct $\mathrm{RF}$, it has two indirect positive $\mathrm{RF}$ components (Derwent et al., 2001; Forster et al., 2007a).

$\mathrm{NO}_{x}$ emissions increase the levels of $\mathrm{OH}$ thereby reducing the levels of $\mathrm{CH}_{4}$ and other gases removed by $\mathrm{OH}$; thus they cause negative $\Delta F . \mathrm{NO}_{x}$ also causes positive $\Delta F$ by production of tropospheric $\mathrm{O}_{3}$ (together with $\mathrm{HO}_{x}$ and $\mathrm{RO}_{x}$ species from $\mathrm{CO}$ and hydrocarbons, under the influence of solar radiation). As discussed in Section 4.2, the effect on $\mathrm{CH}_{4}$ and $\mathrm{O}_{3}$ depends strongly on the region and season of emission. $\mathrm{NO}_{x}$ emissions also cause negative $\Delta F$ via formation of nitrate aerosols and via methane-induced changes in $\mathrm{O}_{3}$.

Organic carbon aerosols (OC), which cause negative $\Delta F$ by reflection of solar radiation, are either emitted directly (primary organic aerosols, POA) or formed in the atmosphere by emissions of semivolatile organic carbon compounds or VOCs that form semi-volatile oxidation products (secondary organic aerosols, SOA). The main precursors of SOA are biogenic (e.g. terpenes), but the SOA formation is affected by anthropogenic emissions by changes in POA (which SOA condenses onto), and by changes in oxidation levels $\left(\mathrm{OH}, \mathrm{O}_{3}\right.$, and $\left.\mathrm{NO}_{3}\right)$. $O C$ also causes negative $\Delta F$ by changing cloud properties.

Black carbon (BC) emissions cause positive $\Delta F$ by absorbing shortwave radiation and by decreasing the albedo when deposited on snow surfaces. The direct forcing is significantly dependent on assumptions about how BC aerosols are mixed with other components of the aerosol population, with internal mixing tending to accentuate the positive forcing (e.g. Forster et al., 2007a). BC also causes a complex set of impacts on cloudiness, including the socalled semi-direct effect, whereby changes in temperature and humidity structure due to the absorption of solar radiation by $\mathrm{BC}$, alter the structure of clouds.

$\mathrm{SO}_{2}$ forms sulphate particles that reflect solar radiation causing negative $\Delta F$. Sulphate particles further cause negative $\Delta F$ by changing cloud properties.
Current climate policies focus on the long-lived greenhouse gases (LLGHGs) (sometimes these are called well-mixed greenhouse gases), which have relatively well-known behaviour and $\Delta F$. However, there is strong evidence that the other emissions and mechanisms play an important role for the transport sector (e.g. Eyring et al., 2005; Lauer et al., 2007; Fuglestvedt et al., 2008). Quantifying these effects is a complex scientific task due to the broad mix of substances and physical/chemical processes involved. The adjustment times are short for many of the emissions associated with modes of transport: months for $\mathrm{O}_{3}$, days for sulphate, $\mathrm{BC}$ and $\mathrm{OC}$ and hours or less for contrails. By contrast, the LLGHGs have adjustment times of decades $\left(\mathrm{CH}_{4}\right)$ and centuries $\left(\mathrm{N}_{2} \mathrm{O}\right.$ and $\left.\mathrm{CO}_{2}\right)$. Thus, an evaluation of the climate impact of transport depends on how future effects in a long-term perspective are evaluated (see Section 5.1). Such an evaluation involves value judgements since very different effects occurring at different times, and affecting different generations, have to be compared and weighted.

Furthermore, transport emissions cause both negative and positive forcing and this raises a fundamental question for policymaking: How should negative forcings (cooling) be treated? One option is simply to ignore them. It may also be argued that cooling effects can be seen as a climate disturbance, e.g. through effects of circulation patterns and the hydrological cycle; thus the absolute value of the $\Delta F$ (or $\Delta T)$ could be used instead. Another option, which is perhaps attractive when a sector (e.g. aviation) is a small contributor to the overall increasing total anthropogenic climate forcing (and therefore does not affect the sign of that forcing), is to take the net impact of cooling and heating effects (with a further desire to account for any regional differences in the net impact notwithstanding). Thus, designing and comparing all these effects on a common scale is a challenging task that involves exercising value judgements (in particular weighting of effects over time).

A consequence of the different lifetimes/adjustment times is that their contributions to climate change differ significantly after the time of emissions. Fig. 5a shows the contribution to global-mean temperature change in 2000 for various emissions from the transport sector for different emission periods since 1900 . This is calculated using a UD-EBM (see Section 3) and the QUANTIFY transport emission 
a

Transport emission contributions for total temperature change in year 2000

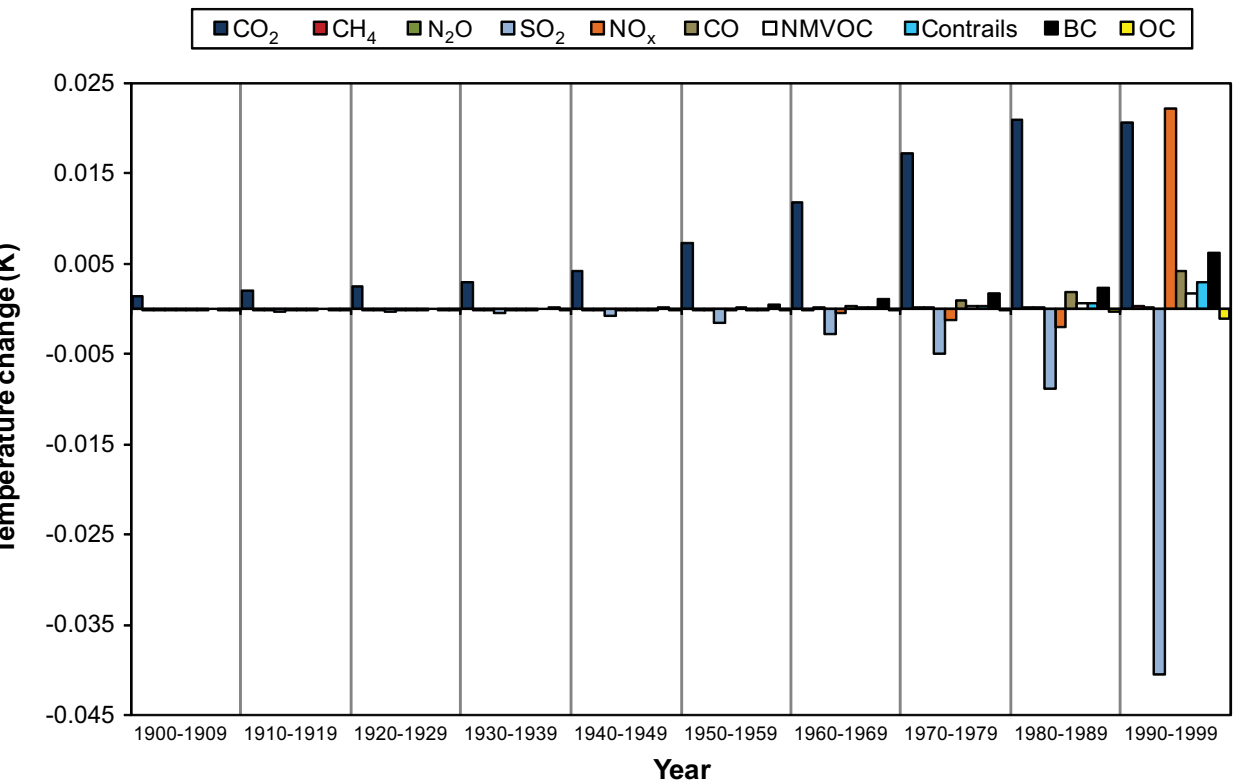

b Transport emission contributions for total temperature change in year 2000

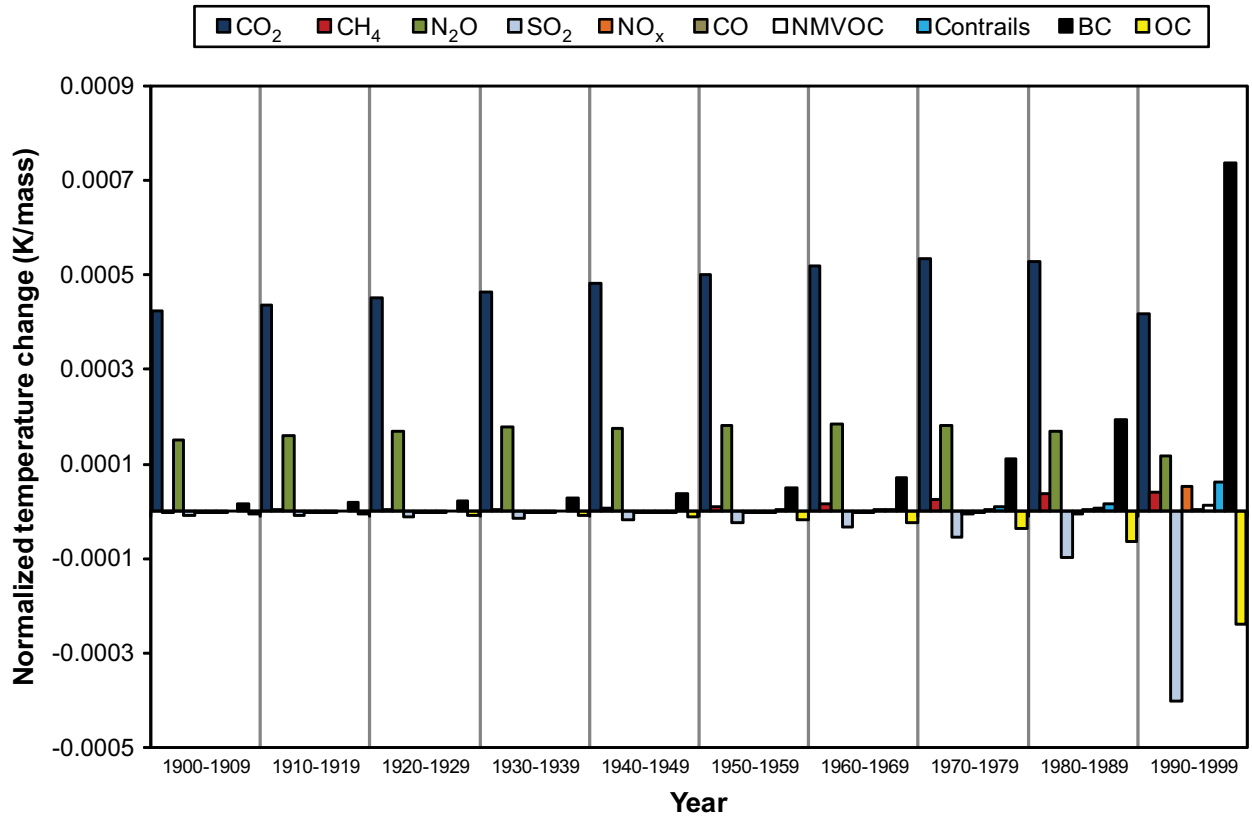

Fig. 5. a) The contribution from various emissions from the transport sector in various periods to the globally-averaged temperature change in 2000 . The CICERO SCM has been used for these calculations with emission numbers from QUANTIFY. ( $\mathrm{SO}_{2}$ effects include direct and first indirect effect.) b) Same as a) but normalized to emissions of the gases during the periods. Contrails are normalized to $\mathrm{CO}_{2}$ emissions as a proxy for fuel use. Units: $\mathrm{CO}_{2}$ and contrails: $\mathrm{K} / \mathrm{PgCO}_{2} ; \mathrm{NO}_{x}: \mathrm{K} \mathrm{TgNO}_{2} ; \mathrm{SO}_{2}$ : $\mathrm{K} / \mathrm{TgS}$; other components: $\mathrm{K} / \mathrm{Tg}$ of component.

inventories. The three determinants of the contribution to temperature change in 2000 are i) the emission histories, ii) the adjustment times of the forcing agents and iii) the thermal inertia of the climate system. For the emission history of the transport sector, it is only $\mathrm{CO}_{2}$ among the early emissions that has caused a non-negligible contribution to the temperature increase in 2000. It is worth noticing that the temperature effect of $\mathrm{NO}_{x}$ switches sign. The $\mathrm{NO}_{x}$ emissions in the period 1950-1990 have a cooling effect on current climate through methane and methane-induced ozone changes. Emissions in the most recent period have a large warming effect through the short-lived mode of ozone, although we emphasize again that the balance between the effect of the different components of the $\mathrm{NO}_{x}$-induced forcing may not apply at spatial scales below the global scale.
In order to remove the effect of emission history we have normalized the contributions by dividing the effect by the mass of emissions in each period (Fig. 5b). We can see that the contribution from the most recent period is smaller than the previous period due to the thermal inertia in the climate system. The short-lived components become important during the most recent periods. The contributions from $\mathrm{CO}_{2}$ are dominant and show (remarkably) little variation over time. This can be explained by the nature of the rise and decay of $\mathrm{CO}_{2}$ perturbations. The effect of $\mathrm{CO}_{2}$ emissions on surface temperature increases rapidly during the first 10-20 years following an emission due to the thermal inertia of the earthatmosphere system. This can be seen in Fig. 5b by the fact that recent emissions have, per $\mathrm{kg}$ emitted, less impact than earlier 

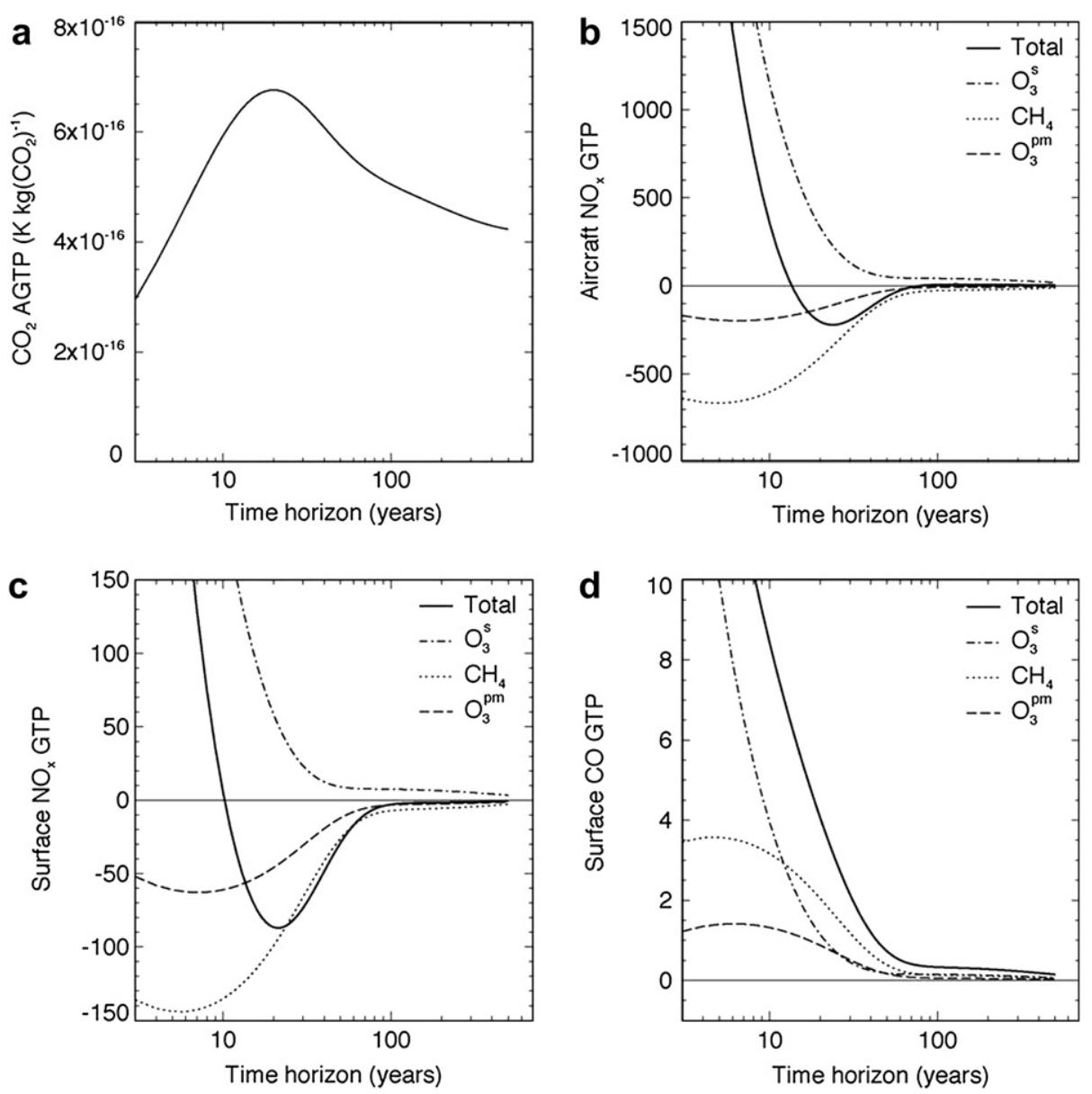

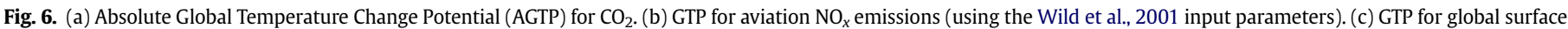
$\mathrm{NO}_{x}$ emissions (using the Wild et al., 2001 input parameters). (d) GTP for mid-latitude surface CO emissions (using the Berntsen et al., 2005 "UiO" input parameters).

emissions - see also Fig. 6a which shows the AGTP for a pulse emission of $\mathrm{CO}_{2}$. The effect of emissions more than 30 years ago on current temperature, diminishes due to the removal of the emitted $\mathrm{CO}_{2}$ from the atmosphere, but this effect is rather gradual; a significant fraction of the emitted $\mathrm{CO}_{2}$ remains in the atmosphere for many centuries (Archer et al., 1997; Joos et al., 1996).

\section{Climate metric values for transport-related emissions}

This section gives numerical values for the GWP and GTP, for the emissions from the transport sector. For short-lived species, the global-mean radiative forcing, which is the basis for both the GWP and the GTP estimates, is based on results from global model simulations published in assessment reports or in the peerreviewed literature. However, the published results are generally not in a form for direct use in metrics (at least not for the GTP), so some further analysis has been done to facilitate the calculations. Some studies present results that are sector specific (e.g. Lauer et al., 2007 for shipping emissions and Stevenson et al., 2004 for aviation) and these results are used for calculations of sectorspecific metrics. For road transport, however, we know of no studies that present the effects of the components individually and studies for anthropogenic emissions in general have been used.

For the short-lived species, the metric values will, in principle, depend on the location of the emissions (e.g. see Section 5.1). Whenever possible we give metric values for mid-latitude and tropical regions separately (i.e. if there are results available from studies that have distinguished between emissions at different latitudes). Also for $\mathrm{NO}_{x}$ we give separate estimates for emissions from aircraft. In principle, a much finer horizontal and vertical resolution is desirable - for example, the emission of $\mathrm{NO}_{x}$ by shipping in a clean mid-latitude environment will have a distinctly different impact to the same emission in a more polluted midlatitude urban environment. Hence, the work here should be regarded as a first step in establishing more refined metrics. More detailed calculations from global models, which will facilitate the next steps, are likely to become available in the coming years. It would be preferable if the input values for metric calculations were derived from multi-model intercomparisons adopting the same methodology - some of the spread in metric values discussed below undoubtedly originates from differences in experimental design (e.g. assumptions regarding time and location, steady-state versus transient model runs, etc), rather than a lack of understanding in the underlying physical processes. Thus, we have not performed any coherent and thorough analysis of effect of uncertainties in input parameters across metrics and components. We do however use information that is available to indicate effects of critical assumptions and the magnitudes of the uncertainties. When several estimates are available (as for $\mathrm{NO}_{x}$ ) we give ranges for the metric values based on the sets of studies. Since the climate sensitivity parameter represents a key factor in the calculation of GTPs we reported, in Section 5.4 , a brief sensitivity test to show how variations in $\lambda$ affect the GTP for components of varying lifetimes.

For GWP we present values for the three time horizon given in the IPCC and WMO assessment reports. However, we recognise that other choices are also defensible and that there is no scientific reason 
for choosing the three used here. For GTP we have chosen to present 20,50 and 100 years. The reason for this is that it represents the likely timescale over which the global-mean temperature would approach a specified target, should such a climate policy be widely adopted. To convey some information about the temporal behaviour of the GTP we choose to use 3 time horizons; however, as will be demonstrated, there can be a large variation in values before and after these times particularly for some short-lived species such as $\mathrm{NO}_{x}$.

\subsection{Long-lived greenhouse gases}

The GWPs for long-lived greenhouse gases have recently been assessed (Forster et al., 2007a) and we repeat the values given in that report in Table 2 for a selection of gases. The AGWPs for $\mathrm{CO}_{2}$ are $2.47 \times 10^{-14}, 8.69 \times 10^{-14}$ and $28.6 \times 10^{-14} \mathrm{Wm}^{-2} \mathrm{yr}\left(\mathrm{kgCO}_{2}\right)^{-1}$ for 20,100 and 500 years respectively. Forster et al. (2007a) give an uncertainty of $\pm 15 \%$ (on the $90 \%$ level) for the AGWP for $\mathrm{CO}_{2}$. Based on the specific forcings and lifetimes for the long-lived gases given in this recent assessment, the GTP values can be readily calculated following the method in Appendix 2.

The decay of a perturbation of atmospheric $\mathrm{CO}_{2}$ following a pulse emission is more complicated than for the other long-lived greenhouse gases (see Section 5.3). Following Forster et al. (2007a), the decay of a pulse emission at time $t$ is approximated by:

$R(t)=a_{0}+\sum_{i=1}^{3} a_{i} \exp \left(-\frac{t}{\alpha_{i}}\right)$

where the parameter values and the analytical expression for the AGTP resulting from the use of this expression are given in Appendix 2. Using these parameters, the AGTP values for $\mathrm{CO}_{2}$ are then $6.8 \times 10^{-16}, 5.8 \times 10^{-16}$, and $5.1 \times 10^{-16} \mathrm{~K} \mathrm{~kg}\left(\mathrm{CO}_{2}\right)^{-1}$ for 20,50 and 100 years respectively. Fig. 6 a shows the time variation of the $\mathrm{CO}_{2}$ AGTP, emphasizing that it is a complex function of time, because it depends on both the time dependence of the pulse of $\mathrm{CO}_{2}$ and the climate system response to that pulse.

Table 2 also presents the GTP values for the same gases; values for other gases can readily be derived from values of specific forcing given in Forster et al. (2007a) and Equation (A4). The indirect effects of methane are included by the using adjustment time instead of the lifetime (to account for the effect on $\mathrm{OH}$ and thereby its own lifetime) and by increasing the specific forcing by a factor 1.4 , to account for effects on tropospheric ozone and stratospheric water vapour (see Forster et al., 2007a).

The GTP values vary with time horizon in a way that depends on adjustment time and how this compares with the adjustment time of $\mathrm{CO}_{2}$. For the short-lived gases (e.g. HCFC-123), the GTP falls rapidly with time horizon. By contrast, the longer-lived gases, such as $\mathrm{N}_{2} \mathrm{O}$ and CFC-12 show a GTP that increases from time horizons of 20-50 years, before decreasing, while for the very long-lived gases (SF 6 , PFC-14 and PFC-116), the GTP continues to increase out to 100 years. The GTP(500) values are higher than for the GTP(100) values for the very long-lived PFCs and $\mathrm{SF}_{6}$. In the case of very short-lived species, the GTP(500) is about $45 \%$ of the GTP(100) value (as will be shown later (Fig. 6) for $\mathrm{NO}_{x}$ and $\mathrm{CO}$ ), while for species with lifetimes of a few decades, the corresponding value can be as low as $15 \%$.

Hence, if a policy goal was to avoid a given amount of warming at some specified time in the future, the GTP(50) and GTP(100) indicate that it would initially be best to concentrate on the longerlived climate agents, with the shorter-lived species becoming more important as the specified time is approached (Shine et al., 2007) this was one of the major concerns of Manne and Richels (2001) in their criticism of the GWP.

The contrast between the GWP and GTP values in Table 2 is particularly noticeable for gases with short lifetimes - for example, for methane, the GWP drops by an about a factor of 3 between time horizons of 20 and 100 years, whereas the GTP drops by an order of magnitude. This reflects the fact that the integral nature of the GWP means that it retains the memory of the strong short-lived forcing; by contrast, the GTP, being an end-point metric, retains less of a memory, as the impact of the pulse in forcing on temperature has largely died out after 100 years.

\subsection{Ozone pre-cursors}

GWPs for $\mathrm{NO}_{x}, \mathrm{CO}$ and VOCs have been published in several studies, while so far no estimates of GTPs are known to have been presented. One study gives GWP(100) for hydrogen $\left(\mathrm{H}_{2}\right)$ (Derwent et al., 2001).

Table 2

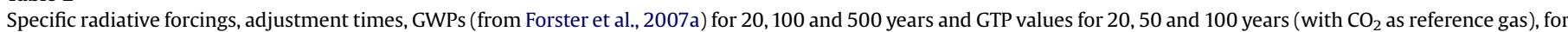

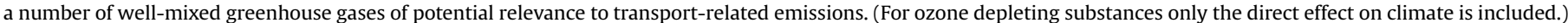
In all tables, for example, $1.46 \mathrm{E}-13$ denotes $1.46 \times 10^{-13}$, etc. The GTP values are specific to a given value of climate sensitivity - see Appendix 2 .

\begin{tabular}{|c|c|c|c|c|c|c|c|c|}
\hline & \multirow[t]{2}{*}{ Specific forcing $\left(\mathrm{Wm}^{-2} \mathrm{~kg}^{-1}\right)$} & \multirow[t]{2}{*}{ Adjustment time (years) } & \multicolumn{3}{|l|}{ GWP } & \multicolumn{3}{|l|}{ GTP } \\
\hline & & & $H=20$ & $H=100$ & $H=500$ & $H=20$ & $H=50$ & $H=100$ \\
\hline $\mathrm{CH}_{4}$ (incl indirect) & $1.82 \mathrm{E}-13$ & 12 & 72 & 25 & 7.6 & 57 & 12 & 4 \\
\hline $\mathrm{N}_{2} \mathrm{O}$ & $3.88 \mathrm{E}-13$ & 114 & 289 & 298 & 153 & 303 & 322 & 265 \\
\hline HFC-23 & $1.53 \mathrm{E}-11$ & 270 & 12000 & 14800 & 12200 & 12800 & 15600 & 16000 \\
\hline HFC-125 & $1.08 \mathrm{E}-11$ & 29 & 6350 & 3500 & 1100 & 6050 & 3370 & 1130 \\
\hline HFC-134a & 8.83E-12 & 14 & 3830 & 1430 & 435 & 3140 & 795 & 225 \\
\hline HFC-152a & 7.67E-12 & 1.4 & 437 & 124 & 38 & 149 & 22 & 18 \\
\hline CFC-11 & $1.02 \mathrm{E}-11$ & 45 & 6730 & 4750 & 1620 & 6710 & 5050 & 2440 \\
\hline CFC-12 & $1.49 \mathrm{E}-11$ & 100 & 11000 & 10900 & 5200 & 11500 & 11800 & 9200 \\
\hline CFC-113 & $9.01 \mathrm{E}-12$ & 85 & 6540 & 6130 & 2700 & 6770 & 6660 & 4820 \\
\hline HCFC-22 & $1.30 \mathrm{E}-11$ & 12 & 5160 & 1810 & 549 & 4100 & 871 & 275 \\
\hline HCFC-123 & $5.15 \mathrm{E}-12$ & 1.3 & 273 & 77 & 24 & 91 & 14 & 11 \\
\hline HCFC-124 & $9.08 \mathrm{E}-12$ & 5.8 & 2070 & 609 & 185 & 1220 & 140 & 87 \\
\hline HCFC-141b & $6.74 \mathrm{E}-12$ & 9.3 & 2250 & 725 & 220 & 1640 & 258 & 106 \\
\hline HCFC-142b & $1.12 \mathrm{E}-11$ & 18 & 5490 & 2310 & 705 & 4840 & 1680 & 439 \\
\hline $\mathrm{CCl}_{4}$ & 4.76E-12 & 26 & 2700 & 1400 & 435 & 2540 & 1290 & 397 \\
\hline $\mathrm{CH}_{3} \mathrm{Br}$ & $5.93 \mathrm{E}-13$ & 0.7 & 17 & 5 & 1 & 5 & 1 & 1 \\
\hline $\mathrm{CH} 3 \mathrm{CCl} 3$ & $2.53 \mathrm{E}-12$ & 5 & 506 & 146 & 45 & 277 & 31 & 21 \\
\hline $\mathrm{H}-1211$ & $1.02 \mathrm{E}-11$ & 16 & 4750 & 1890 & 575 & 4080 & 1240 & 328 \\
\hline H-1301 & $1.21 \mathrm{E}-11$ & 65 & 8480 & 7140 & 2760 & 8660 & 7760 & 4840 \\
\hline $\mathrm{SF}_{6}$ & $2.00 \mathrm{E}-11$ & 3200 & 16300 & 22800 & 32600 & 17500 & 23400 & 28000 \\
\hline PFC-14 & $6.40 \mathrm{E}-12$ & 50000 & 5210 & 7390 & 11200 & 5620 & 7560 & 9180 \\
\hline PFC-116 & $1.06 \mathrm{E}-11$ & 10000 & 8630 & 12200 & 18200 & 9300 & 12500 & 15100 \\
\hline
\end{tabular}


In principle calculations of both GWP and GTP require knowledge of the time-evolution of the net radiative forcing $(\Delta F(t))$ following these emissions (i.e. short-lived ozone, and methane and methane-induced ozone perturbations as discussed in Section 6.1). Two methods have been applied for calculation of these GWPs, both based on CTM or CCM simulations. The first category (later called transient) follows the evolution (concentration and forcing) of a pulse emission (Derwent et al., 2001; Stevenson et al., 2004; Wild et al., 2001), while the second approach (later called steady-state) performs steady-state simulations (Berntsen et al., 2005; Endresen et al., 2003; Eyring et al., 2007; Fuglestvedt et al., 1999; Köhler et al., 2008; Naik et al., 2005). The studies using the transient approach present integrated $\Delta F$ (or AGWPs) and usually the corresponding methane perturbation lifetime. With the steady-state approach, the $\Delta F$ for the steady-state perturbations are calculated, and to derive the AGWPs and AGTPs corresponding to pulse emissions, additional information about the lifetimes of the short-lived ozone perturbation and the methane perturbation, must be included in the analysis. This method by which this is done is explained in Appendix 2.

The impact of an emission pulse depends on time of the year (e.g. Derwent et al., 2008) but for use in policymaking the impact of annual averaged emissions seems most relevant. For $\mathrm{NO}_{x}$ we therefore do not use results from studies that only treat a pulse emission for a given time of the year (e.g. Derwent et al., 2008) and only include results that are basically showing the effect of one-year step emissions pulses (Stevenson et al. (2004) report the average over several months) or from continuous emission changes.

To put the reported results into a common mathematical framework consistent with one-year emission pulses we assume temporal evolutions of ozone and methane as described below:

For the short-lived ozone perturbation we assume

$\Delta F_{\mathrm{O}_{3}}^{S}(t)=\Delta F_{\mathrm{O}_{3}}^{S, S S}\left(1-\exp \left(-t / \alpha_{S}\right)\right)$ for $t<1$

$\Delta F_{\mathrm{O}_{3}}^{S}(t)=\Delta F_{\mathrm{O}_{3}}^{S, \mathrm{SS}}\left(1-\exp \left(-1 / \alpha_{S}\right)\right) \exp \left(-(t-1) / \alpha_{S}\right)$ for $t \geq 1$

where $\alpha_{S}$ is the short-lived lifetime and the superscript SS indicates "steady-state". These expressions are derived by assuming that the pulse lasts for one year and hence there are constant emissions during year 1 (Equation (10)) followed by a decay of the end-of-year 1 concentrations from then onwards (Equation (11)). Here the value of 0.267 years is chosen for $\alpha_{s}$, but the results are insensitive to the small variations around this value.

For the methane perturbation we assume

$\Delta F_{\mathrm{CH}_{4}}(t)=\Delta F_{\mathrm{CH}_{4}}^{\mathrm{SS}_{4}}\left(1-\exp \left(-t / \alpha_{P M}\right)\right) \quad$ for $t<1$

$\Delta F_{\mathrm{CH}_{4}}(t)=\Delta F_{\mathrm{CH}_{4}}^{\mathrm{SS}}\left(1-\exp \left(-1 / \alpha_{P M}\right)\right) \exp \left(-(t-1) / \alpha_{P M}\right)$ for $t \geq 1$

where $\alpha_{P M}$ is the primary-mode lifetime (effectively the adjustment time of methane) due to the effect of $\mathrm{NO}_{x}$ on methane and methane-induced ozone. Values are given in Table 3.

For the methane-induced ozone perturbation, we assume

$\Delta F_{\mathrm{O}_{3}}^{P M}(t)=\Delta F_{\mathrm{O}_{3}}^{P M, S S}\left(1-\exp \left(-1 / \alpha_{P M}\right)\right) \exp \left(-(t-1) / \alpha_{P M}\right)$ for $t \geq 1$

(The very small contribution from the methane-induced ozone perturbation in year 1 is assumed to be included in the short-lived $\mathrm{O}_{3}$ response.)
With knowledge of the short-lived and methane and methaneinduced ozone lifetimes, the corresponding $\Delta F$ for steady-state perturbations can be calculated (Table 3 ). Then the AGWP and AGTP values for the ozone pre-cursors are calculated using the expressions in Appendix 2. The GWP and GTP are then presented by taking ratios with the AGWP and AGTP for $\mathrm{CO}_{2}$.

\subsection{Main features of the metric values for ozone pre-cursors}

Table 4 presents the net GWPs and GTPs for $\mathrm{NO}_{x}, \mathrm{CO}$, and VOC and the components which contribute to these net values; i.e. short-lived ozone, and methane and methane-induced ozone perturbations. The magnitudes and the temporal behaviour of the effects vary significantly between the studies included here.

The $\mathrm{NO}_{x}$ results are split between aircraft sources, surface sources (themselves split between tropics and mid-latitudes) and shipping. The aviation sources could be further divided by altitude region of emissions (Grewe and Stenke, 2008 (although note that their results are for emissions in 2050 assuming a scenario of future emissions); Köhler et al., 2008) and all sources could be further subdivided by region of emission, but we do not do so here. The general pattern for $\mathrm{NO}_{x}$ is that the short-lived ozone forcing is always positive, while the methane-induced ozone forcing and methane forcing are always negative.

For $\mathrm{NO}_{x}$ from aircraft, all the net GWPs (Table 4a) are positive for $H=20$ years, but there is a large spread in results (a factor of 4 between Köhler et al., 2008 and Stevenson et al., 2004). Even where the net GWP agrees, the individual components can disagree substantially. The net effect depends on the balance of the individual components, which themselves depend on the forcings and lifetimes. For $H=100$ years, one study gives a negative net GWP for aircraft, while the other studies give positive values ranging from 7 to 71 . For $\mathrm{NO}_{x}$ from surface sources, five of the GWP estimates are positive and two are negative for $H=20$ years, while for $H=100$ and 500 years all, except one study, give negative values. Shipping $\mathrm{NO}_{x}$ emissions are strongly negative at all time horizons; this is because of the low $\mathrm{NO}_{x}$ environment into which ships generally emit $\mathrm{NO}_{x}$. From the available studies it is not possible to draw strong conclusions on the difference in the net GWP between surface emissions in midlatitudes and the tropics, although clearly the short-lived ozone component is stronger in the tropics.

The GTP values for $\mathrm{NO}_{x}$ in Table $4 \mathrm{~b}$ contrast significantly with the GWPs. The GTPs are all uniformly negative for 20 and 50 years, because the methane and methane-induced ozone effects dominate. The fact that the two different metric approaches can generate values which differ in sign is a striking illustration of the need for careful choice of metric approach, and the need to choose a metric which is appropriate for the climate policy being pursued.

The time variation of the $\mathrm{NO}_{x}$ GTPs can be somewhat counterintuitive - it might be anticipated that the long-lived negative forcing from the methane decrease would yield a more negative value of GTP at longer time horizons, and yet Table $4 \mathrm{~b}$ indicates that, for example, using the parameters for the aviation case from Wild et al. (2001), the GTP(20) is negative and yet the GTP(100) is positive. Fig. $6 \mathrm{~b}$ shows the time variation for this case study both for the total and the individual components. It emphasizes several important points. Firstly, the time variation of the total GTP can be quite complex, a fact that is obscured by the somewhat arbitrary choices of time horizon in Table 4b. Hence, while $\operatorname{GTP}(20)$ is negative, the GTP becomes strongly positive for $\mathrm{H}$ of 10 years and less. Secondly, the total GTP is a very fine balance between the three contributing terms. The system retains a memory of the very strong positive, short-lived ozone forcing which is sufficient to offset the effects of longer-lived negative forcings at 100 years. In order to 
Table 3

Derived steady-state radiative forcings for sustained emissions of $1 \mathrm{~kg} \mathrm{yr}^{-1}$ of $\mathrm{NO}_{x}, \mathrm{CO}$ or VOCs, and primary-mode (methane) adjustment time.

\begin{tabular}{|c|c|c|c|c|}
\hline & $\Delta F_{\mathrm{O}_{3}}^{\mathrm{SS}}\left(\mathrm{Wm}^{-2}\left(\mathrm{~kg}(\mathrm{~N}) \text { year }^{-1}\right)^{-1}\right)$ & $\Delta F_{\mathrm{O}_{3}}^{P M}\left(\mathrm{Wm}^{-2}\left(\mathrm{~kg}(\mathrm{~N}) \text { year }^{-1}\right)^{-1}\right)$ & $\Delta F_{\mathrm{CH}_{4}}^{\mathrm{SS}}\left(\mathrm{Wm}^{-2}\left(\mathrm{~kg}(\mathrm{~N}) \text { year }^{-1}\right)^{-1}\right)$ & $\alpha_{P M}(\mathrm{yr})$ \\
\hline \multicolumn{5}{|l|}{$\overline{\text { Aircraft } N O_{x}}$} \\
\hline Stevenson et al. (2004) & $1.66 \mathrm{E}-11$ & $-3.12 \mathrm{E}-12$ & $-1.38 \mathrm{E}-11$ & 11.5 \\
\hline $\begin{array}{l}\text { Wild et al. (2001) } \\
\text { (as in Stevenson et al. (2004) }\end{array}$ & $2.60 \mathrm{E}-11$ & $-4.92 \mathrm{E}-12$ & $-1.51 \mathrm{E}-11$ & 11.8 \\
\hline Köhler et al. (2008) & $4.41 \mathrm{E}-11$ & $-1.62 \mathrm{E}-11$ & $-2.79 \mathrm{E}-11$ & 14.0 \\
\hline \multicolumn{5}{|l|}{ Surface $\mathrm{NO}_{x}(\mathrm{pr} . \mathrm{kg} \mathrm{N})$} \\
\hline Naik et al. (2005) Tropics & $1.38 \mathrm{E}-11$ & $-4.83 \mathrm{E}-12$ & $-1.16 \mathrm{E}-11$ & 12.6 \\
\hline Wild et al. (2001) Tropics & $1.49 \mathrm{E}-11$ & $-5.13 \mathrm{E}-12$ & $-1.08 \mathrm{E}-11$ & 14.2 \\
\hline Naik et al. (2005) Mid-lat & $8.02 \mathrm{E}-13$ & $-7.12 \mathrm{E}-13$ & $-1.69 \mathrm{E}-12$ & 12.6 \\
\hline Berntsen et al. (2005) UiO, Mid-lat & $2.78 \mathrm{E}-12$ & $-7.80 \mathrm{E}-13$ & $-1.90 \mathrm{E}-12$ & 10.8 \\
\hline Berntsen et al. (2005) LMDz, Mid-lat & $3.20 \mathrm{E}-12$ & $-1.10 \mathrm{E}-12$ & $-2.69 \mathrm{E}-12$ & 16.1 \\
\hline Wild et al. (2001) Mid-lat & $2.00 \mathrm{E}-12$ & $-9.43 \mathrm{E}-13$ & $-1.90 \mathrm{E}-12$ & 14.2 \\
\hline Wild et al. (2001) Global & $4.59 \mathrm{E}-12$ & $-1.79 \mathrm{E}-12$ & $-3.80 \mathrm{E}-12$ & 14.2 \\
\hline \multicolumn{5}{|l|}{ Shipping $N \mathrm{O}_{x}(\mathrm{pr} . \mathrm{kg} \mathrm{N})$} \\
\hline Eyring et al. (2007) & $3.16 \mathrm{E}-12$ & $-1.88 \mathrm{E}-12$ & $-4.52 \mathrm{E}-12$ & 12.2 \\
\hline Endresen et al. (2003) & 7.99E-12 & $-3.20 \mathrm{E}-12$ & $-7.71 \mathrm{E}-12$ & 10.2 \\
\hline Fuglestvedt et al. (2008) & 7.19E-12 & $-1.88 \mathrm{E}-12$ & $-7.56 \mathrm{E}-12$ & 10.2 \\
\hline Surface CO & $\Delta F_{\mathrm{O}_{3}}^{S}\left(\mathrm{Wm}^{-2}\left(\mathrm{~kg}(\mathrm{CO}) \mathrm{year}^{-1}\right)^{-1}\right)$ & $\Delta F_{\mathrm{O}_{3}}^{P M}\left(\mathrm{Wm}^{-2}\left(\mathrm{~kg}(\mathrm{CO}) \text { year }^{-1}\right)^{-1}\right)$ & $\Delta F_{\mathrm{CH}_{4}}^{\mathrm{SS}}\left(\mathrm{Wm}^{-2}\left(\mathrm{~kg}(\mathrm{CO}) \text { year }^{-1}\right)^{-1}\right)$ & \\
\hline Berntsen et al. (2005) UiO, Mid-lat & $8.96 \mathrm{E}-14$ & $3.30 \mathrm{E}-14$ & $7.55 \mathrm{E}-14$ & 10.8 \\
\hline Berntsen et al. (2005) LMDz, Mid-lat & $9.33 \mathrm{E}-14$ & $5.52 \mathrm{E}-14$ & $1.40 \mathrm{E}-13$ & 16.1 \\
\hline Derwent et al. (2001) & $6.0 \mathrm{E}-14$ & & $1.1 \mathrm{E}-13$ & 12.3 \\
\hline Surface VOC & $\Delta F_{\mathrm{O}_{3}}^{S}\left(\mathrm{Wm}^{-2}\left(\mathrm{~kg}(\mathrm{VOC}) \text { year }^{-1}\right)^{-1}\right)$ & $\Delta F_{\mathrm{O}_{3}}^{P M}\left(\mathrm{Wm}^{-2}\left(\mathrm{~kg}(\mathrm{VOC}) \text { year }^{-1}\right)^{-1}\right)$ & $\Delta F_{\mathrm{CH}_{4}}^{\mathrm{SS}}\left(\mathrm{Wm}^{-2}\left(\mathrm{~kg}(\mathrm{VOC}) \text { year }^{-1}\right)^{-1}\right)$ & \\
\hline Collins et al. (2002) & $2.13 \mathrm{E}-13$ & & $1.77 \mathrm{E}-13$ & 12.2 \\
\hline
\end{tabular}

illustrate the importance of how the memory of the ocean is treated by the GTP we have also used the simplified form of the GTP (Equation (8)), which neglects the thermal inertia of the deep ocean. In this case the GTP $(100)$ is found to be negative, rather than positive as the system has less "memory" of the strong and positive short-lived effect.

For surface sources, there is more agreement for the different regions (with the tropical emissions giving more negative values at all time horizons) for the GTP than for the GWP. Because of the varying signs, it is difficult to make general statements about the differences between the size of the GWP and GTP values. Nevertheless, it can be seen that, as with the shorter-lived greenhouse gases discussed in Section 7.1, between 20 and 100 years, the individual components of the GWP values typically decrease by a factor of 3 or 5 while the GTP the decrease is by more than an order of magnitude. This reflects the fact that the GWP integrates forcing along the path and hence retains information on the effect of the pulse at early times, whereas the GTP is an end-point metric, where the impact of the early effect of the pulse is much less at longer time horizons. Fig. $6 c$ shows the time-evolution of the GTP for this case, using the parameters the global surface emissions case from Wild et al. (2001). It shows generally similar behaviour to the aircraft case (Fig. 6b) but with a somewhat different balance between the individual terms.

Derwent et al. (2008) calculated GWPs for $\mathrm{NO}_{x}$ emissions for one-month pulses at various locations within the Asian continent and found strong dependence on the magnitude, location and season of emissions. In all cases they found negative net GWPs for a 100-year time horizon. For 21 selected regions in Asia their median (net) GWP(100) was -10 .

It is tempting to conclude, from the widespread of $\mathrm{NO}_{x}$ values for a given region of emissions in Table 4, that it is the metrics themselves that are at fault. However, (and notwithstanding the fact that some of the differences are very likely due to experimental design) all the metrics are doing is reflecting the level of understanding of the net effects of $\mathrm{NO}_{x}$ emissions and the lack of agreement amongst the results derived from the much more sophisticated models. We regard it as a strength of the metrics that they are able to illustrate, in a simple manner, the level of understanding. While the spread between studies (scientific uncertainty) can be expected to decrease in the future, the difference between the metrics is of a different nature and reflects the differences in purpose and underlying structure of the metrics. Likewise, the spreads between different time horizons are in line with the characteristics of the components and the climate system.

For CO, all three forcings (short-lived ozone, methane and methane-induced ozone) are positive. There are relatively few studies available and we find GWP values roughly between 1 and 10. Fig. $6 \mathrm{~d}$ shows the time variation of the GTP, which emphasizes how distinct it is from $\mathrm{NO}_{x}$ as the sign of the methane and methaneinduced ozone effects are the same as the short-lived effect on ozone; the Figure also emphasizes how quickly the GTP increases for time horizons less than 20 years. For VOCs, the GWP values (from one study only) are 14 and 5, for 20 and 100 years, about a factor of six lower than the methane values. As with $\mathrm{NO}_{x}$, it is clear that the GTP value falls off much more strongly with increasing time horizon than does the GWP (i.e. by around an order of magnitude rather than a factor of 3 between time horizons of 20 and 100 years). The metric values for VOC given here are constructed based on radiative forcing estimates for emissions of 6 anthropogenic VOC species from Collins et al. (2002), and the emissions weight factors for these species from IPCC (2001). Thus $1 \mathrm{~kg}$ VOC is assumed to consist of $0.11 \mathrm{~kg}$ ethane, $0.13 \mathrm{~kg}$ propane, $0.25 \mathrm{~kg}$ butane, $0.34 \mathrm{~kg}$ ethylene, $0.06 \mathrm{~kg}$ propylene, and $0.11 \mathrm{~kg}$ of toluene. (Collins et al., 2002 present GWP values for 10 individual VOCs and not aggregated as presented here.)

We do not provide values for $\mathrm{H}_{2}$ - a candidate for future fuel - but Derwent et al. (2001) report values of GWP(100) of 3.4 for the effect on methane and 2.4 from the effect on ozone, giving a total of 5.8 .

\subsection{Aerosols}

A number of atmospheric models have been developed over the last decade to include all major aerosols affecting climate. These include sulphate (from $\mathrm{SO}_{2}$ emissions), carbonaceous aerosols (black carbon and organic carbon) and nitrate particles. A number 
Table 4

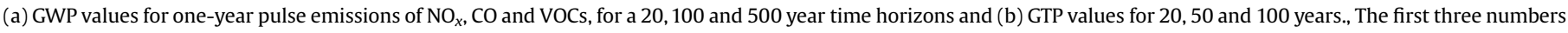

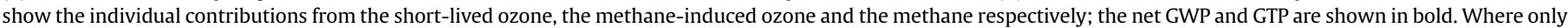

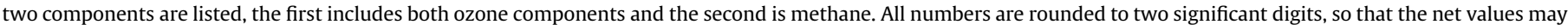

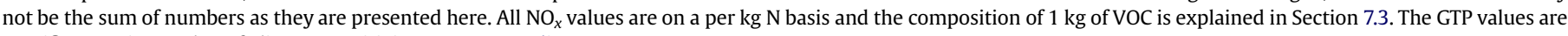
specific to a given value of climate sensitivity - see Appendix 2.

\begin{tabular}{|c|c|c|c|}
\hline \multirow[t]{2}{*}{ (a) } & \multicolumn{3}{|l|}{ GWP } \\
\hline & $H=20$ & $H=100$ & $H=500$ \\
\hline $\begin{array}{l}\text { Aircraft } \mathrm{NO}_{x}(\mathrm{pr} . \mathrm{kg} \mathrm{N}) \\
\text { Stevenson et al. } \\
\text { Wild et al. (in Stevenson et al.) } \\
\text { Kohler et al. } \\
\text { Range of Net }\end{array}$ & $\begin{array}{l}670-100-460=120 \\
1100-150-490=410 \\
1800-470-850=470 \\
120 \text { to } 470\end{array}$ & $\begin{array}{l}190-34-160=-2.1 \\
300-54-170=71 \\
510-180-320=6.9 \\
-2.1 \text { to } 71\end{array}$ & $\begin{array}{l}58-10-48=-0.7 \\
91-17-53=22 \\
154-55-98=2.0 \\
-0.7 \text { to } 22\end{array}$ \\
\hline $\begin{array}{l}\text { Surface } \mathrm{NO}_{x}(\mathrm{pr} . \mathrm{kg} \mathrm{N}) \\
\text { Naik et al. Tropics } \\
\text { Wild et al. Tropics } \\
\text { Range of Net }\end{array}$ & $\begin{array}{l}560-150-370=43 \\
600-150-330=130 \\
43 \text { to } 130\end{array}$ & $\begin{array}{l}160-53-130=-28 \\
170-57-120=-9.7 \\
-28 \text { to }-9.7\end{array}$ & $\begin{array}{l}48-16-41=-8.5 \\
52-17-38=-3.0 \\
-8.5 \text { to }-3.0\end{array}$ \\
\hline $\begin{array}{l}\text { Naik et al. Mid-lat } \\
\text { Berntsen et al. UiO, Mid-lat } \\
\text { Berntsen et al. LMDz, Mid-lat } \\
\text { Wild et al. Mid-lat } \\
\text { Range of Net }\end{array}$ & $\begin{array}{l}33-22-54=-43 \\
110-25-64=23 \\
130-30-76=23 \\
81-27-57=-3.7 \\
-43 \text { to } 23\end{array}$ & $\begin{array}{l}9.2-7.9-19=-18 \\
32-8.6-22=1.6 \\
37-12-31=-6.3 \\
37-12-31=-9.3 \\
-18 \text { to } 1.6\end{array}$ & $\begin{array}{l}2.8-2.4-5.9=-5.5 \\
9.7-2.6-6.6=0.5 \\
11.2-3.7-9.4=-1.9 \\
7.0-3.2-6.6=-2.8 \\
-5.5 \text { to } 0.5\end{array}$ \\
\hline Wild et al. Global & $190-52-110=19$ & $53-20-44=-11$ & $16.0-6.0-13=-3.3$ \\
\hline $\begin{array}{l}\text { Shipping } \mathrm{NO}_{x}(\mathrm{pr} . \mathrm{kg} \mathrm{N}) \\
\text { Eyring et al. } \\
\text { Endresen et al. } \\
\text { Fuglestvedt et al. } \\
\text { Range of Net }\end{array}$ & $\begin{array}{l}130-60-150=-76 \\
320-100-270=-47 \\
290-61-260=-31 \\
-76 \text { to }-31\end{array}$ & $\begin{array}{l}36-21-52=-36 \\
92-35-89=-32 \\
83-21-87=-25 \\
-36 \text { to }-25\end{array}$ & $\begin{array}{l}11-6.3-16=-11 \\
28-11-27=-9.7 \\
25-6.3-26=-7.6 \\
-11 \text { to }-7.6\end{array}$ \\
\hline $\begin{array}{l}\text { Surface CO } \\
\text { Berntsen et al. UiO, Mid-lat } \\
\text { Berntsen et al. LMDz, Mid-lat } \\
\text { Derwent et al. }\end{array}$ & $\begin{array}{l}3.6+1.1+2.5=7.2 \\
3.8+1.5+4.0=9.3 \\
2.4+3.5=6.0\end{array}$ & $\begin{array}{l}1.0+0.4+0.9=2.3 \\
1.1+0.6+1.6=3.3 \\
0.7+1.3=2.0\end{array}$ & $\begin{array}{l}0.3+0.1+0.3=0.7 \\
0.3+0.2+0.5=1.0 \\
0.2+0.4=0.6\end{array}$ \\
\hline $\begin{array}{l}\text { Surface VOC } \\
\text { Collins et al. }\end{array}$ & $8.6+5.7=14$ & $2.5+2.0=4.5$ & $0.7+0.6=1.4$ \\
\hline
\end{tabular}

(b)

GTP

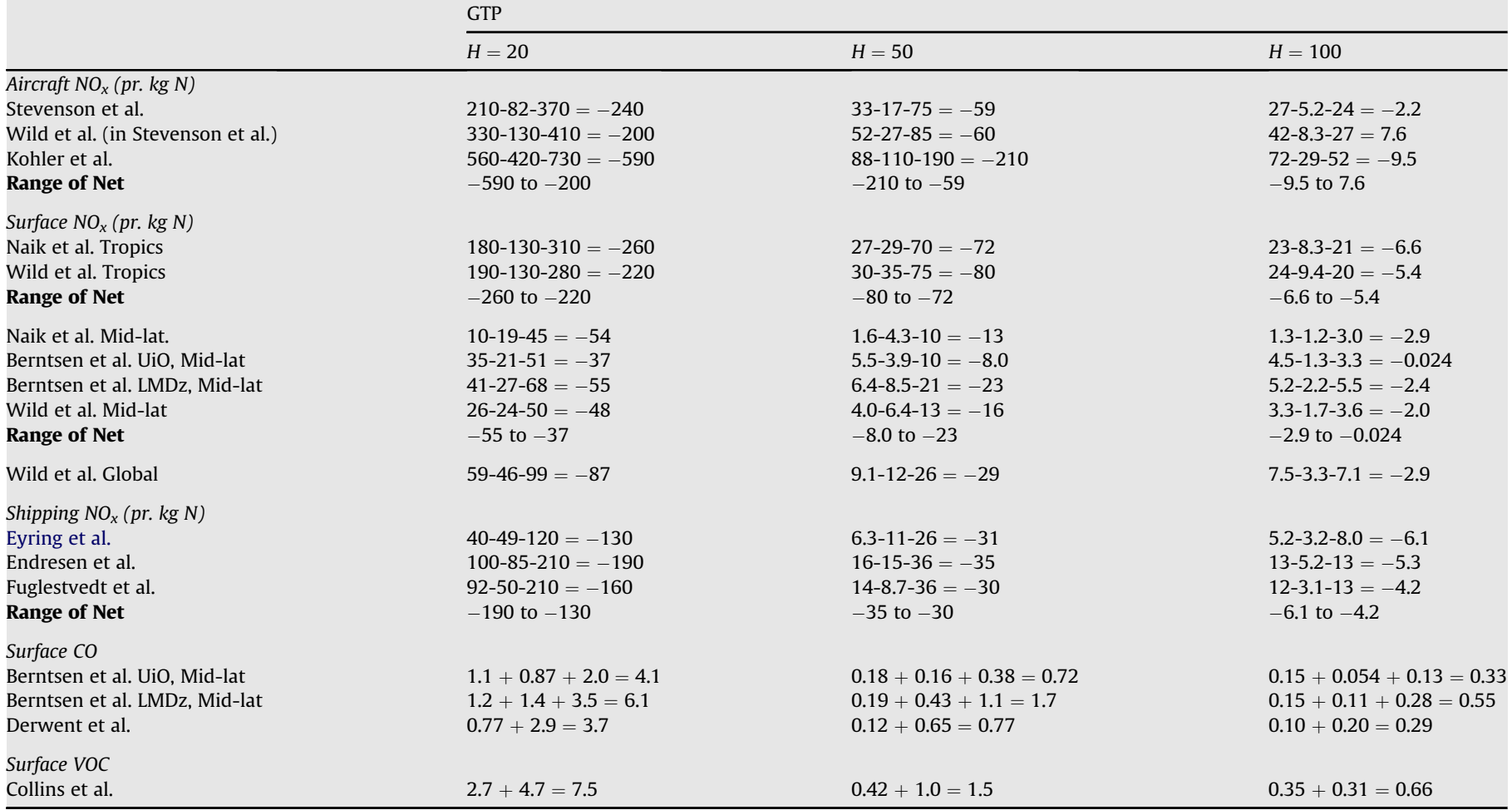


of published studies have also focused on the effects of emissions from different regions, thus making it possible to derive regional metric values (Koch et al., 2007; Naik et al., 2007; Reddy and Boucher, 2007). Each of these studies provides either tables or diagrams of equilibrium radiative forcing values, and lifetimes, for each region. However, an examination of the output from these models (see Appendix 3 ) reveals that there is not a robust relationship between the region of emission and the metric value hence, regions that yield the highest metric value in one study, do not, in general, do so in the other studies. This could be because of fundamental differences in the representations of atmospheric processes in the models, or this may simply represent differences in the experimental design - as noted above, a tight intercomparison, would be required to ascertain the relative importance of these explanations. Hence, we conclude that it would be premature to present regional values, and restrict the main discussion to globalmean results derived from the mean of a multi-model intercomparison. Appendix 3 does, at least, indicate the possible size of the effects from regional variations.

Schulz et al. (2006) provide an intercomparison of radiative forcing values for a number of different aerosol types, for 9 different models; the results for the mean values from these models are used here. The values of forcing and lifetime will be specific to the particular distribution of emissions adopted in this intercomparison. Aerosols have direct effects (direct absorption/scattering of mainly solar radiation), but also indirect effects through modification of cloud properties or altering surface albedo (e.g. black carbon being deposited on snow and ice). Generally, the indirect effects are much less certain than the direct effects (Forster et al., 2007a), and the data from published studies are thus mainly for the direct effect, with the exception of shipping emissions - see below. One crude approach is just to multiply the direct effect by some highly uncertain factor, perhaps of order 1.5 to 2 (Forster et al., 2007a) to take the indirect effect into account. We have not adopted that approach here, although it would be straightforward for the reader to do so.

To calculate GWPs and GTP data for the direct effect, specific radiative forcing $\left(\mathrm{Wm}^{-2} \mathrm{~kg}^{-1}\right)$ and atmospheric residence time is needed. For $\mathrm{SO}_{2}$ this is more complicated, as we require the fraction of $\mathrm{SO}_{2}$ that leads to the sulphate $\left(\mathrm{SO}_{4}\right)$ that contributes to the aerosol forcing. Further, it is the lifetime of $\mathrm{SO}_{4}$ that is required, rather than the lifetime of $\mathrm{SO}_{2}$. Hence, the specific forcing in this case is $\mathrm{Wm}^{-2}\left(\mathrm{~kg} \text { of } \mathrm{SO}_{2} \text { that contributes to the } \mathrm{SO}_{4} \text { burden }\right)^{-1}$. In practice, this is derived from the steady-state aerosol forcings available in the literature by dividing that forcing by the annual emissions of $\mathrm{SO}_{2}$ multiplied by the lifetime of $\mathrm{SO}_{4}$.

Table 5 summarises the GWP $(20,100$ and 500 year time horizons) and GTP (20, 50 and 100 years) for pulse emissions of BC, OC and $\mathrm{SO}_{2}$, respectively, based on the results from Schulz et al. (2006). The lifetimes are 7.4, 7.6 and 4 days, respectively. All sulphur values are on an $\mathrm{SO}_{2}$ basis, $\mathrm{BC}$ on a carbon basis and all OC are on an organic carbon basis (not organic matter) with a ratio of particulate organic matter to organic carbon assumed to be 1.4

Table 5 shows a number of generic features (see also Section 7.3). First, despite their very short lifetimes (of order one week), the GWP(100) values are nevertheless large, ranging from -40 to more than 450 times more powerful than $\mathrm{CO}_{2}$, most markedly for black carbon. Second, and importantly, the difference between the GWP and GTP values is substantial and illustrates again why, in a policy context, the use of metrics must rest on a conscious choice and must be appropriate to the policy being pursued. The $\operatorname{GTP}(100)$ is typically a factor of 6 smaller than the GWP(100); as GTP(100) is an end-point metric, at such time horizons, the climate system will have less memory of the impact of a short-lived pulse.

The values in Schulz et al. (2006) are derived from a multimodel comparison from which some idea of uncertainties can be inferred. The standard deviation of the lifetimes, expressed as a percentage of the mean value, is around $25-30 \%$. The spread in specific forcings (and the metric values will scale linearly with changes these forcings) is larger, ranging from $24 \%$ for sulphate to $51 \%$ for OC. The actual uncertainty in BC is likely to be higher, because of its sensitivity to assumptions in the aerosol mixing (see Section 6).

Table 6 gives GWP and GTP values for the shipping sector based on recent studies of this sector (Endresen et al., 2003; Eyring et al., 2005; Fuglestvedt et al., 2008; Lauer et al., 2007), for both the direct and indirect effect of sulphur emissions (we assume the same value of $\mathrm{SO}_{4}$ lifetime as given by Schulz et al. (2006) in these calculations and this assumption has a negligible effects on the results since the lifetime is so short). The direct GWP(100) values range from -11 to -43 . The indirect GWPs and GTPs are typically 10 times larger than the direct GWP values, but the uncertainty in the indirect GWP is likely to be much larger than for the direct. Only one study (Lauer et al., 2007) has so far reported detailed calculations of the indirect forcing specifically for this sector and, further, they include the albedo and lifetime indirect effects; the uncertainty in the lifetime effect is particularly large (Forster et al., 2007a). Their total indirect forcing is several times larger than that found by Capaldo et al. (1999) and Fuglestvedt et al. (2008) who adopted simpler methods. As shown in Table 6, (Lauer et al., 2007) also find a wide spread of values depending on the emission inventory used. The contrast between the GTPs and GWPs is as was discussed earlier for the direct effect.

\subsection{Contrails and aviation-induced cirrus}

Recent studies have looked at either the impact of heightdependent (but latitudinally-independent) perturbations to the present-day fleet for contrails (Rädel and Shine, 2008) or the height and latitudinally dependent perturbations (relative to a 2050 background), with particular attention to a possible supersonic fleet (Grewe and Stenke, 2008; Stenke et al., 2007).

According to the model results of Stenke et al. (2007), less than $2 \%$ of the present day total contrail radiative forcing arises from altitudes outside of $8-12 \mathrm{~km}$ and most of this is within the latitude band $30^{\circ} \mathrm{N}$ to $90^{\circ} \mathrm{N}$. Within this band and height region, specific contrail forcing (i.e. forcing per given distance flown) is $1.4 \mathrm{mWm}^{-2} 10^{9} \mathrm{~km}^{-1}$. This result is broadly consistent with Rädel and Shine (2008) who find a peak in the global-mean specific contrail forcing of $0.37 \mathrm{mWm}^{-2} 10^{9} \mathrm{~km}^{-1}$ for flights at about $10 \mathrm{~km}$. If future fleets were to fly in the $16-20 \mathrm{~km}$ region in the tropics, they would also have a significant impact, as this region has a high degree of ice-supersaturation and hence a high potential contrail cover.

Table 7 shows the GWP and GTP values for contrails using the integrated forcings from Forster et al. (2007b). Since, as noted in Section 6, there is no direct relationship between an aviation emission and a consequent contrail forcing, it is less clear how to reference the emissions in terms of metrics. Here we relate the total forcing due to contrails to the total aviation emissions of $\mathrm{CO}_{2}$ by the aviation fleet and calculate the AGWP and AGTP on a per $\mathrm{kg}\left(\mathrm{CO}_{2}\right)$ basis. An alternative, which is more consistent with the way aircraft generate contrails, would be to present them on a per km flown basis, but this would not then be consistent with the mass-based metrics used for the other emissions. Table 7 shows that despite the short lifetime of contrails (here $5 \mathrm{~h}$ is chosen), the $\operatorname{GWP}(100)$ remains non-negligible, because the initial forcing is so large. It must be stressed that these values are only suitable for use for the average of the present-day fleet - for the reasons discussed in Section 4.2, they are not appropriate for individual flights and would not be appropriate were there to be significant changes in 
Table 5

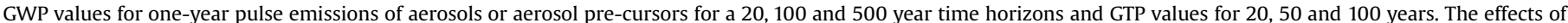

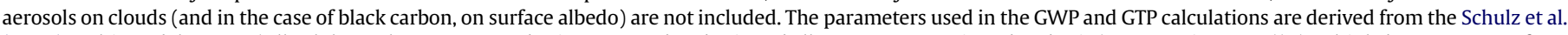

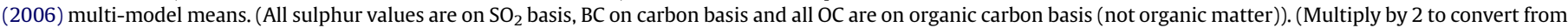
$\mathrm{SO}_{2}$ to $\mathrm{S}$ basis - the lifetimes quoted for sulphate are for $\mathrm{SO}_{4}$.) The GTP values are specific to a given value of climate sensitivity - see Appendix 2 .

\begin{tabular}{|c|c|c|c|c|c|c|c|c|}
\hline \multirow[t]{2}{*}{ Aerosol type } & \multirow[t]{2}{*}{ Specific forcing $\left(\mathrm{Wm}^{-2} \mathrm{~kg}^{-1}\right)$} & \multirow[t]{2}{*}{ Adjustment time (years) } & \multicolumn{3}{|l|}{ GWP } & \multicolumn{3}{|l|}{ GTP } \\
\hline & & & $H=20$ & $H=100$ & $H=500$ & $H=20$ & $H=50$ & $H=100$ \\
\hline Black carbon & $1.96 \mathrm{E}-9$ & 0.020 & 1600 & 460 & 140 & 470 & 77 & 64 \\
\hline Organic carbon & $-2.9 \mathrm{E}-10$ & 0.021 & -240 & -69 & -21 & -71 & -12 & -10 \\
\hline Sulphate & $-3.2 \mathrm{E}-10$ & 0.011 & -140 & -40 & -12 & -41 & -6.9 & -5.7 \\
\hline
\end{tabular}

the altitudes or routes taken by aircraft. The uncertainty in the forcings due to contrails, and hence in the GWPs and GTPs, is of order of 1.5-2 (Forster et al., 2007a; Rädel and Shine, 2008).

Table 7 also presents values for aviation-induced cirrus (AIC), based on a simple scaling that the forcing is about three times that of contrails (Forster et al., 2007a). As noted in Section 3, there are formidable difficulties in calculating the forcing due to AIC with any confidence, and the uncertainty is of order a factor of 3 (Forster et al., 2007a).

\subsection{Aviation water vapour}

Table 8 shows the specific radiative forcing and lifetimes due to water vapour emissions at 3 different height regions and two latitude regions (Grewe and Stenke, 2008). At pressures greater than $500 \mathrm{hPa}$ (i.e. below roughly $6 \mathrm{~km}$ ), the forcing is assumed to be negligible. The water vapour lifetime is a strong function of pressure, so that aviation water vapour emissions above the tropopause cause a greater accumulation per unit emission. A systematic assessment of the uncertainties is not available; we estimate that tropospheric lifetimes to be uncertain by around $20 \%$ and stratospheric lifetimes to be uncertain by around $40 \%$. The uncertainty in specific forcing is most likely dominated by the assumed vertical distribution of the water vapour change, and may be several 10's of percent.

Table 9 shows the resulting GWP and GTP values. The results reflect the same trends as seen in Table 8 , with metric values increasing rapidly as the altitude of emission increases, particularly in the tropics. They show that for aircraft flying well into the stratosphere ( $89 \mathrm{hPa}$ is around $17 \mathrm{~km}$ or $55 \mathrm{kft}$ ), the $\operatorname{AGWP}(100)$ is greater than that for $\mathrm{CO}_{2}$; given that, when burning kerosene, about $0.4 \mathrm{~kg}$ of water vapour is emitted per $\mathrm{kg}$ of $\mathrm{CO}_{2}$, the $\mathrm{CO}_{2}$-equivalent emissions from $\mathrm{CO}_{2}$ and $\mathrm{H}_{2} \mathrm{O}$ are going to be of roughly similar size. However, as with the other short-lived species discussed above, the $\operatorname{GTP}(100)$ values are smaller, but still, for GTP(20), the values are of the same magnitude as those for $\mathrm{CO}_{2}$. This supports the IPCC (1999) conclusion, that for any future fleets of high-flying supersonic aircraft, the effect of water vapour emissions is likely to be a significant, or even the dominant, contributor to their climate forcing.

We are unaware of any estimates in the literature which provide specific forcings and lifetimes for the present-day fleet as a whole. Applying the specific forcings and lifetimes in Table 8 for 30-90N and $198 \mathrm{hPa}$, which is broadly the region of peak emissions for the present-day fleet, would yield a radiative forcing of $3.6 \mathrm{mWm}^{-2}$, somewhat higher than the estimate of $2.8 \mathrm{mWm}^{-2}$ given by Lee et al. (2010). Hence the GWP and GTP values given in Table 9 for 30$90 \mathrm{~N}$ and $198 \mathrm{hPa}$ can be viewed as a very approximate indication of the GWP and GTP for the present-day fleet.

\subsection{Impact of metric choice on calculated $\mathrm{CO}_{2}$-equivalent emissions}

The fundamentally different nature of the GWP and GTP is evident when these metrics are used to calculate $\mathrm{CO}_{2}$-equivalent emissions. The standard application of metrics for transferring emissions to a common scale $-\mathrm{CO}_{2}$ equivalents, $\mathrm{CO}_{2} \mathrm{eq}(\mathrm{H})$ - is given by:

$\mathrm{CO}_{2} \mathrm{eq}(H)=\mathrm{E}_{i} \times M_{i}(H)$

where $i$ is the gas, $M$ is the metric and $H$ is the chosen time horizon.

Fig. 7 shows calculated global man-made emissions in terms of $\mathrm{CO}_{2}$ equivalents when GWP and GTP for some chosen time horizons are applied. Fig. 7 uses the Wild et al. (2001) surface global-mean values from Table 4 for $\mathrm{NO}_{x}$, and the global-mean values for aerosols given in Table 5 (i.e. neglecting the indirect effects); it must be recognised that for these and all other short-lived components there is a significant uncertainty in the GWP and GTP values, as discussed above.

The large differences between the two metrics as well as between the chosen time horizons are striking. Due to the memory

Table 6

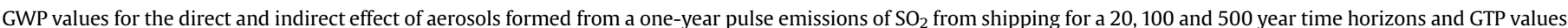

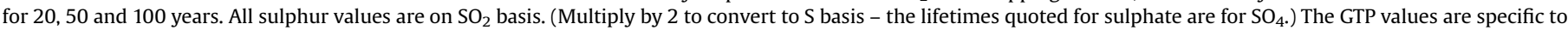
a given value of climate sensitivity - see Appendix 2. The three values presented for Lauer et al. (2007) are for different emission inventories.

\begin{tabular}{|c|c|c|c|c|c|c|c|c|}
\hline \multirow[t]{2}{*}{ Study } & \multirow[t]{2}{*}{ Specific forcing $\left(\mathrm{Wm}^{-2} \mathrm{~kg}^{-1}\right)$} & \multirow[t]{2}{*}{ Lifetime (years) } & \multicolumn{3}{|l|}{ GWP } & \multicolumn{3}{|l|}{ GTP } \\
\hline & & & $H=20$ & $H=100$ & $H=500$ & $H=20$ & $H=50$ & $H=100$ \\
\hline \multicolumn{9}{|l|}{ Direct } \\
\hline Endresen et al. & $-2.70 \mathrm{E}-10$ & 0.011 & -120 & -34 & -10 & -35 & -5.7 & -4.8 \\
\hline Eyring et al. & $-1.66 \mathrm{E}-10$ & 0.011 & -73 & -21 & -6 & -21 & -3.5 & -2.9 \\
\hline Lauer et al. & $-8.75 \mathrm{E}-11$ & 0.011 & -37 & -11 & -3 & -11 & -1.8 & -1.5 \\
\hline Fuglestvedt et al. & $-3.43 \mathrm{E}-10$ & 0.011 & -150 & -43 & -13 & -44 & -7.3 & -6.1 \\
\hline \multicolumn{9}{|l|}{ Indirect } \\
\hline Lauer et al. A & $-3.54 \mathrm{E}-09$ & 0.011 & -1600 & -440 & -130 & -450 & -75 & -63 \\
\hline Lauer et al. B & $-1.72 \mathrm{E}-09$ & 0.011 & -760 & -220 & -66 & -220 & -37 & -31 \\
\hline Lauer et al. C & $-3.30 \mathrm{E}-09$ & 0.011 & -1500 & -410 & -130 & -420 & -70 & -58 \\
\hline
\end{tabular}


Table 7

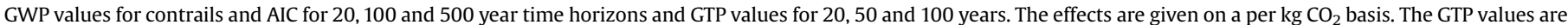
specific to a given value of climate sensitivity - see Appendix 2 .

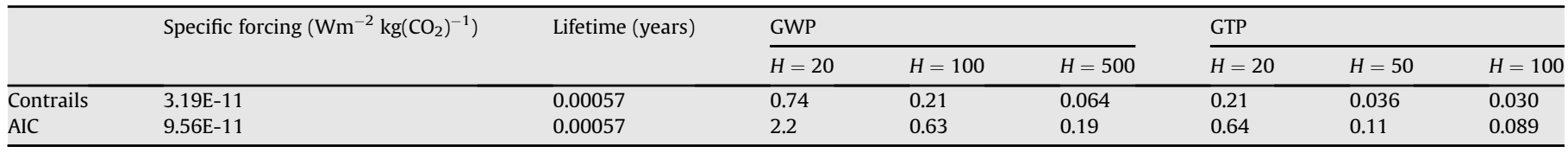

of integrated RF up to the chosen time horizon, the GWP-based perspective gives a much larger relative weight to the short-lived components. Even with 100-year time horizon the short-lived components give a significant contribution. For the GTP based calculation of $\mathrm{CO}_{2}$ eq the effects of short-lived are only evident for $H=20$ years, while after 50 years $\mathrm{CH}_{4}$ and $\mathrm{N}_{2} \mathrm{O}$ are the only non$\mathrm{CO}_{2}$ gases that contribute significantly.

\subsection{Relative level of understanding in metric values}

There are large variations in the level of scientific understanding across the metric values for the various components. As mentioned, this reflects the general level of understanding and the ability to model and quantify the effects of these emissions. Below we present a rough ranking based on our assessment of current level of understanding and ability to quantify the effects, starting with the most certain.

The components for which we have the best estimates are the (almost) inert LLGHGs; such as $\mathrm{N}_{2} \mathrm{O}$, and hydrofluorocarbons like HFC-134a. There are few, if any, indirect effects that have a significant impact on radiative forcing although there are some uncertainties in lifetimes and radiative efficiency.

Then follows the chemically or biologically active LLGHGs; $\mathrm{CO}_{2}, \mathrm{CH}_{4}$, CFC-12, HCFC-22. For $\mathrm{CO}_{2}$ there are uncertainties in our understanding and quantification of the carbon cycle and the exchange of carbon with the reservoirs. For methane there are important uncertainties due to indirect effects on tropospheric $\mathrm{O}_{3}$, stratospheric $\mathrm{H}_{2} \mathrm{O}$ and on its own lifetime. For CFC-12, HCFC22 and other CFCs and HCFCs, there are still significant uncertainties related to the effects on stratospheric $\mathrm{O}_{3}$ and the resulting $\Delta F$.

The next category consists of $\mathrm{CO}$ and water vapour from aviation. For $\mathrm{CO}$ there are significant uncertainties related to chemical effects on tropospheric $\mathrm{O}_{3}$ and $\mathrm{OH}$ (thereby $\mathrm{CH}_{4}$ and other gases removed by $\mathrm{OH}$ ). For $\mathrm{H}_{2} \mathrm{O}$ from aviation, the impact depends mostly on the height above the tropopause at which the water vapour is injected - the position of the tropopause is highly variable in time (on timescales from days to seasons) and location.

The next group is the VOCs which is not a well-defined group of hydrocarbons. They have complex chemical effects on tropospheric $\mathrm{O}_{3}$ and $\mathrm{OH}$ (and thereby $\mathrm{CH}_{4}$ and other gases removed by $\mathrm{OH})$. This group of gases is treated differently across model experiments, since various numbers of gases are lumped together

\section{Table 8}

Specific radiative forcing values for water vapour (in $\mathrm{Wm}^{-2} \mathrm{~kg}\left(\mathrm{H}_{2} \mathrm{O}\right)^{-1}$ ) and lifetimes for emissions in 3 different height regions and two latitude regions (Grewe and Stenke, 2008).

\begin{tabular}{lllllll}
\hline & \multicolumn{3}{l}{$\begin{array}{l}\text { Specific radiative forcing } \\
\left(\mathrm{Wm}^{-2} \mathrm{~kg}\left(\mathrm{H}_{2} \mathrm{O}\right)^{-1}\right)\end{array}$} & & \multicolumn{3}{l}{ Lifetime (years) } \\
& 52 & 89 & 198 & 52 & 89 & 198 \\
\hline Pressure $(\mathrm{hPa})$ & $2.89 \mathrm{E}-13$ & $3.07 \mathrm{E}-13$ & $2.13 \mathrm{E}-13$ & 1.28 & 0.83 & 0.08 \\
$30^{\circ} \mathrm{N}-90^{\circ} \mathrm{N}$ & $2.86 \mathrm{E}-13$ & $3.19 \mathrm{E}-13$ & $2.00 \mathrm{E}-13$ & 2.13 & 0.73 & 0.01 \\
$30^{\circ} \mathrm{S}-30^{\circ} \mathrm{N}$ & & & & & &
\end{tabular}

in different ways or some representative key species are modelled explicitly.

The next group is $\mathrm{SO}_{2}$, $\mathrm{OC}$ aerosols and contrails. Both direct effects and especially indirect cloud effects are very uncertain. Forster et al. (2007a) give a medium level of scientific understanding of the direct effects and low for the indirect effects on clouds. The level of understanding of contrails is also regarded as low, partly because information on the amount and properties of contrail on a global-scale is not yet available and partly because the net forcing is a small residual of the sum of shortwave cooling and longwave warming.

$\mathrm{NO}_{x}, \mathrm{BC}$ aerosols and aviation-induced cirrus form the final category. Estimates of the climate forcing caused by $\mathrm{NO}_{x}$ are very dependent on experimental design, and model treatment of transport processes. As shown in Section 7.3, the net effect of responses in short-lived ozone, methane and methane-induced ozone shows large variation amongst available calculations. There are also large uncertainties related to $\mathrm{BC}$, both due to strong dependence on location and model treatment of the direct, semidirect and indirect effects, and the snow albedo effect. Forster et al. (2007a) give medium-low for the level of scientific understanding of $\mathrm{BC}$ on snow. For AIC there are serious difficulties in distinguishing natural and AIC from observations and, to date, most studies have had to rely on empirical relationships between air traffic and cirrus trends. In addition, as with contrails, the tight balance between shortwave cooling and longwave warming makes the net effect uncertain.

\section{Ozone depletion metrics relevant for the transport sector}

Ozone in the stratosphere, between about 15 and $30 \mathrm{~km}$ altitude, protects the Earth's surface from harmful ultraviolet radiation from the sun (UNEP, 2007). Since the end of the 1970s, the ozone layer has been depleted by anthropogenic emissions of ozone depleting substances (ODSs) with halocarbons having the greatest impact (WMO, 2007). Several metrics are in use to

\section{Table 9}

GWP values for aircraft emissions of water vapour at different altitudes of emission for 20, 100 and 500 year time horizons and GTP values for 20,50 and 100 years Based on data from Grewe and Stenke (2008). $52 \mathrm{hPa}, 89 \mathrm{hPa}$ and $198 \mathrm{hPa}$ are approximately $20 \mathrm{~km}, 17 \mathrm{~km}$ and $12 \mathrm{~km}$, respectively. The GTP values are specific to a given value of climate sensitivity - see Appendix 2 .

\begin{tabular}{|c|c|c|c|c|c|c|}
\hline & \multicolumn{3}{|c|}{ GWP $\left(30^{\circ} \mathrm{N}-90^{\circ} \mathrm{N}\right)$} & \multicolumn{3}{|c|}{$\operatorname{GTP}\left(30^{\circ} \mathrm{N}-90^{\circ} \mathrm{N}\right)$} \\
\hline & $H=20$ & $H=100$ & $H=500$ & $H=20$ & $H=50$ & $H=100$ \\
\hline $52 \mathrm{hPa}$ & 15 & 4.3 & 1.3 & 5.0 & 0.75 & 0.60 \\
\hline $89 \mathrm{hPa}$ & 10 & 2.9 & 0.89 & 3.3 & 0.51 & 0.42 \\
\hline \multirow[t]{3}{*}{$198 \mathrm{hPa}$} & 0.69 & 0.20 & 0.059 & 0.20 & 0.033 & 0.028 \\
\hline & \multicolumn{3}{|c|}{ GWP $\left(30^{\circ} \mathrm{S}-30^{\circ} \mathrm{N}\right)$} & \multicolumn{3}{|c|}{$\operatorname{GTP}\left(30^{\circ} \mathrm{S}-30^{\circ} \mathrm{N}\right)$} \\
\hline & $H=20$ & $H=100$ & $H=500$ & $H=20$ & $H=50$ & $H=100$ \\
\hline$\overline{52 \mathrm{hPa}}$ & 25 & 7.0 & 2.1 & 9.3 & 1.27 & 1.0 \\
\hline $89 \mathrm{hPa}$ & 9.4 & 2.7 & 0.81 & 3.0 & 0.46 & 0.38 \\
\hline $198 \mathrm{hPa}$ & 0.081 & 0.023 & 0.0070 & 0.024 & 0.0039 & 0.0033 \\
\hline
\end{tabular}




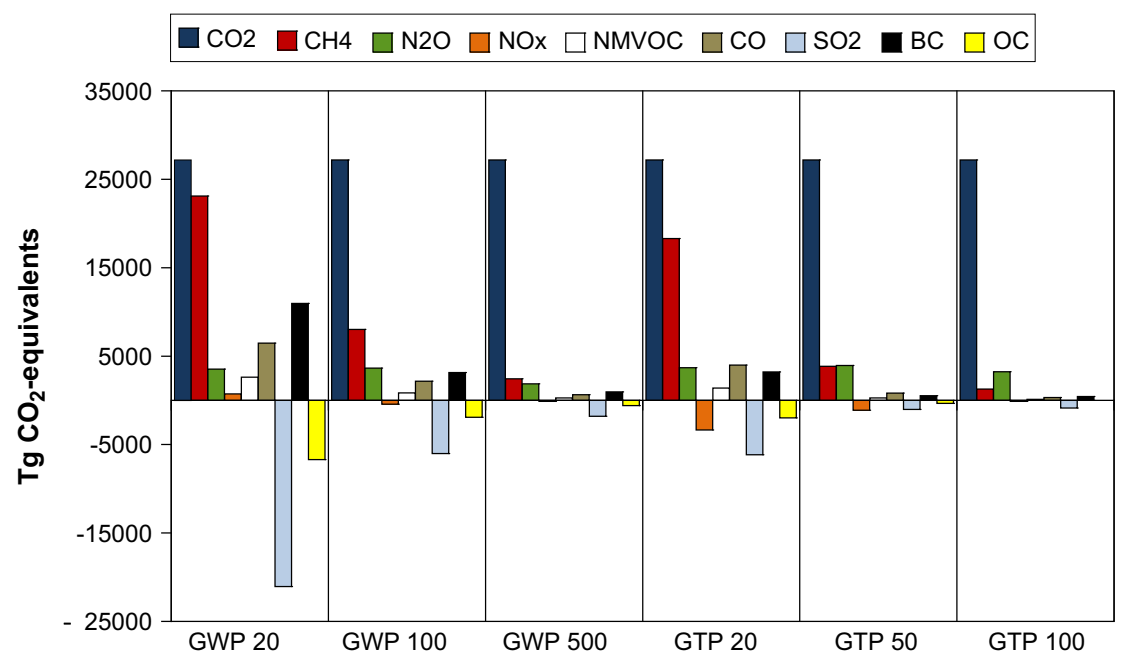

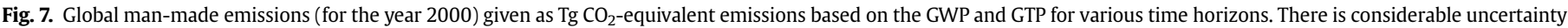
in the values for short-lived species in particular; see text for the discussion of the specific choices made for the GWP and GTP values used in this figure.

compare the effects of different ODSs on the ozone layer or to compare different scenarios. There are no metrics for comparing ODSs that directly incorporate the relationship between ozone concentration and ultraviolet radiation reaching the surface or other environmental effects of ozone depletion such as skin cancer rates. The metrics that are discussed here are: 1) the Ozone Depletion Potential (ODP), which links the emissions of ozone depleting substances to their relative effects on the ozone layer and 2) the Equivalent Effective Stratospheric Chlorine (EESC) which is a measure for the amount of chlorine and bromine in the stratosphere and related to the amount of ozone depletion.

\subsection{Ozone Depletion Potential (ODP)}

The relative contribution of individual halocarbons to stratospheric ozone depletion is often expressed by their ODP (Fisher et al., 1990a; Solomon and Albritton, 1992; Wuebbles, 1983). The metric of ODPs has been used in the 'Scientific Assessments of Ozone Depletion' (WMO, 1999, 2003, 2007) and in the Montreal Protocol on 'Substances that deplete the ozone layer' (ozone.unep. org). Generally, so-called steady-state ODPs are used. They are calculated assuming constant emissions and steady-state conditions. For compounds that are removed by linear processes, this is equivalent to assuming an emission pulse and integrating over the entire decay of the compound (Prather, 1996, 2002). Time-dependent ODPs can also be calculated (Solomon and Albritton, 1992), which reflect the different timescales over which the compound and reference gas (CFC-11) liberate chlorine and bromine into the stratosphere. Compounds that have shorter atmospheric lifetimes than CFC-11 have ODPs that decrease with increasing integration time, and vice versa.

The ODP of a compound $i$ is defined as

$\mathrm{ODP}_{i}=$

global $\mathrm{O}_{3}$ loss due to sustained unit mass emission of $i$ global $\mathrm{O}_{3}$ loss due to sustained unit mass emission of $\mathrm{CFC}-11$.

This formula is usually applied to compounds that are wellmixed in the atmosphere. The ODP can be calculated using detailed chemistry-transport models. Since in the ODP the ozone loss of a compound is expressed relative to the ozone loss of the same emission of CFC-11, it is relatively insensitive to the complexity of the model. Using this property, ODPs can be calculated using a semi-empirical formula as

$\mathrm{ODP}_{i}=\left(n_{\mathrm{Cl}, i}+\alpha n_{\mathrm{Br}, i}\right) \frac{f_{i}}{f_{\mathrm{CFC}-11}} \frac{\tau_{i}}{\tau_{\mathrm{CFC}-11}} \frac{M_{\mathrm{CFC}-11}}{M_{i}} \frac{1}{3}$,

where $\tau$ is the lifetime of the compound, $M$ the molecular weight, $n$ the number of chlorine/bromine atoms in the molecule, and $\alpha$ the higher effectiveness of bromine compared to chlorine for ozone destruction. The relative fractional release, the ratio $f_{i} / f_{\mathrm{CFC}-11}$, is a measure of the fractional release of inorganic halogen compounds relative to the fractional release of CFC-11. Observations in the stratosphere of correlations between halocarbons are used to evaluate the relative stratospheric release of chlorine and bromine and to derive the fractional release values.

Table 10 shows the ODPs given in WMO (2007) of the major halocarbons as calculated with the semi-empirical method and as laid down in the Montreal Protocol.

The ODP is similar to the GWP, as both metrics are used to compare the effects of emissions of a gas relative to the emission of the same amount of a reference gas, $\mathrm{CFC}-11$ or $\mathrm{CO}_{2}$, respectively. A difference between both metrics is that the ODP usually refers to steady-state conditions, while the GWP explicitly refers to a specific time horizon. However, as noted above, the ODP can be interpreted as the impact of a pulse emission integrated over an infinite time horizon, so in this sense can be considered equivalent to the GWP for this choice of time horizon.

Since ODPs are a relative measure they are subject to fewer uncertainties than absolute reductions in stratospheric ozone. Uncertainties in ODPs arise from uncertainties in atmospheric lifetimes and chemical and dynamical processes in the stratosphere. The ODPs from model calculations vary by about $\pm 15 \%$ for CFCs, $\pm 20 \%$ for HCFCs and $\pm 25 \%$ for halons (WMO, 1992). For gases with atmospheric lifetimes of a few years or shorter steady-state ODPs are not appropriate, but time-dependent ODPs should be considered. The ODP of short-lived gases depends also on the location and season of emission.

\subsection{Equivalent Effective Stratospheric Chlorine}

The Equivalent Effective Stratospheric Chlorine (EESC) is a metric for ozone depletion that refers to the state (composition) of the atmosphere and is thereby the analogue to the role of radiative forcing as climate change metric. The EESC index (Daniel et al., 
Table 10

Lifetimes (years) and Ozone Depletion Potentials (relative to CFC-11) for halocarbons (from WMO, 2007).

\begin{tabular}{llll}
\hline Halocarbon & Lifetime (Years) & Semi-empirical ODP & ODP in Montreal Protocol \\
\hline Chlorofluorocarbons & & \\
CFC-11 & 45 & 1.0 & 1.0 \\
CFC-12 & 100 & 1.0 & 1.0 \\
CFC-113 & 85 & 1.0 & 0.8 \\
CFC-114 & 300 & 1.0 & 1.0 \\
CFC-115 & 1700 & 0.44 & 0.6 \\
Halons & & & \\
Halon-1301 & 65 & 16 & 10 \\
Halon-1211 & 16 & 7.1 & 3.0 \\
Halon-2402 & 20 & 12 & 6.0 \\
Halon-1202 & 2.9 & 1.7 & - \\
Hydrochlorofluorocarbons & & \\
HCFC-22 & 12 & 0.05 & 0.055 \\
HCFC-123 & 1.3 & 0.02 & 0.02 \\
HCFC-124 & 5.8 & 0.02 & 0.022 \\
HCFC-141b & 9.3 & 0.12 & 0.11 \\
HCFC-142b & 18 & 0.07 & 0.065 \\
HCFC-225ca & 1.9 & 0.02 & 0.025 \\
HCFC-225cb & 5.8 & 0.03 & 0.033 \\
Others & & & \\
CCl & 26 & 0.73 & 1.1 \\
$\mathrm{CH}_{3} \mathrm{CCl}{ }_{3}$ & 5.0 & 0.12 & 0.1 \\
$\mathrm{CH}_{3}$ Br & 0.7 & 0.51 & 0.6 \\
$\mathrm{CH}_{3} \mathrm{Cl}$ & 1.0 & 0.02 & - \\
\hline
\end{tabular}

1995; WMO, 2007) has been used frequently as a measure of the amount of chlorine and bromine available in the stratosphere to destroy ozone. Contributions of very short-lived chlorine- and bromine-containing source gases and of tropospheric inorganic halogens are generally neglected. EESC is defined as

$$
\begin{aligned}
\operatorname{EESC}(t)= & f_{\mathrm{CFC}-11}\left[\sum_{\begin{array}{c}
\text { Cl-containing } \\
\text { halocarbons }
\end{array}} n_{i} \frac{f_{i}}{f_{\mathrm{CFC}-11}} \rho_{i, \text { entry }}\right. \\
& \left.+\alpha \sum_{\substack{\text { Br-containing } \\
\text { halocarbons }}} n_{i} \frac{f_{i}}{f_{\mathrm{CFC}-11}} \rho_{i, \text { entry }}\right]
\end{aligned}
$$

where $n$ is the number of chlorine or bromine atoms in the source gas, $f_{i} / f_{\text {CFC-11 }}$ represents the efficiency of the stratospheric halogen release relative to that of $\mathrm{CFC}-11$, denoted by $f_{\mathrm{CFC}-11}$, and $\rho_{i \text {,entry }}$ is the tropospheric mixing ratio of source gas $i$ when it entered the stratosphere. Traditionally, $\rho_{i, \text { entry }}$ is calculated assuming a simple time lag $I$ from the surface observations of usually 3 years, typical for the lower mid-latitude stratosphere, i.e.

$\rho_{i, \text { entry }}(t)=\rho_{i}(t-\Gamma)$

where $\rho_{i}(t)$ is the surface mixing ratio at time $t$. Recently, a few changes have been proposed to the formulation of EESC (Newman et al., 2007, 2006) that include a spectrum for the age-of-air applied to the time lag and the fractional release values. This EESC can be applied more appropriately to various parts of the stratosphere and provides quantitative estimates of inorganic chlorine and bromine in the stratosphere. Effective equivalent chlorine (EECl) (Montzka et al., 1996) is a similar quantity to EESC, but includes no consideration of the transport lag time.

It has generally been assumed that if all other atmospheric parameters and processes remain constant, ozone depletion relates linearly to EESC above a certain threshold level. The year EESC returns to its 1980 levels (the threshold level) is used as a metric in WMO Ozone Assessments to compare different scenarios. The year 1980 is chosen because this is the approximate date when midlatitude and Antarctic ozone depletion was observed to begin. An exception to this relationship is Antarctic ozone depletion.
Springtime depletion became so marked around 1990 that there was not enough ozone left in the lower stratosphere for the column ozone amount to continue to follow a linear relationship with EESC. So it is assumed here that no additional Antarctic ozone destruction occurs for EESC values above 1990 levels.

A second metric (WMO, 2007) that has been used to compare scenarios is the integrated EESC value above the 1980 level, integrated from 1980 or the current time until EESC returns to the 1980 level. This metric is meant to represent the cumulative ozone depletion due to ODSs over the specified time frame.

\subsection{Contributions of the transport sector}

The emissions of halocarbons are the main cause of the observed depletion of the ozone layer. Historically, the largest emissions are from refrigeration, air conditioning (stationary and mobile), foam blowing, chemical cleaning, fire extinguishing and soil fumigation. As a consequence of the Montreal Protocol and amendments and adjustments, the production, use and emissions of most ODSs have decreased strongly. The largest current and expected future emissions of ODSs are from CFCs and HCFCs present in existing refrigeration and air conditioning systems and foams, and from future production of HCFCs for refrigeration and air conditioning systems.

The transport sector can affect the ozone layer by, 1) direct effects of the emissions of ODSs from the transport sector on stratospheric chemistry, 2) direct effects of emissions of other species on stratospheric chemistry, 3 ) indirect effects of emissions on tropospheric ozone, temperatures in the stratosphere, circulation changes in the troposphere and stratosphere. Indirect effects are not quantified here as we do not have the necessary information with which to do so.

The direct effects of ODSs on the chemistry of the stratosphere include emissions from:

- HCFCs from transport refrigeration. This consists of refrigeration systems for transporting chilled or frozen goods. Transport takes place by road, rail, air and sea and containers as refrigerated systems are used with moving carriers. Most of the refrigerants banked in transport refrigeration are HCFCs and HFCs. Emissions from systems used in sea transport and fishing emit about 3 ktonnes HCFC-22 annually (IPCC, 2005). This is about $1 \%$ of the total HCFC-22 emission of 231 ktonnes in 2002.

- CFCs from mobile air-conditioners. Mobile air-conditioners initially used CFC-12 as refrigerant, but are now mainly equipped with HFC-134a. The emission of CFC-12 was about $63 \mathrm{kt} \mathrm{yr}^{-1}$ in 2002, but is expected to decrease to about 5

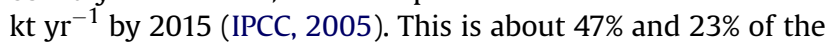
total CFC-12 emission in 2002 and 2015, respectively.

- Methyl bromide $\left(\mathrm{CH}_{3} \mathrm{Br}\right)$ from gasoline. The emission is estimated at about $5 \mathrm{kt} \mathrm{yr}^{-1}$ (WMO, 2003) compared with a total (natural and anthropogenic) emission about $170 \mathrm{kt} \mathrm{yr}^{-1}$ in 2005 (WMO, 2007).

- Halons from fire extinguishers on aircraft. Halons have been used in fire extinguishers for, e.g. engine spaces on ships and aircraft and ground protection of aircraft. Although halons are no longer necessary in virtually any new installations, they are harder to replace by alternatives in, e.g. passenger spaces and cargo compartments of commercial aircraft, and crew compartments of military combat vehicles (IPCC, 2005). Since the ban in production of halons by the Montreal Protocol, only recycled halons are currently used in new and existing systems.

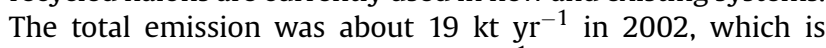
expected to decrease to about $3 \mathrm{kt} \mathrm{yr}^{-1}$ by 2015 . 
The direct effects of other emissions on stratospheric chemistry include:

- Emissions from catalytic converters on cars are a small source of nitrous oxide $\left(\mathrm{N}_{2} \mathrm{O}\right)$ of about $350 \mathrm{kt} \mathrm{yr}^{-1}$, which is $1.5 \%$ of the all anthropogenic emissions and $0.6 \%$ of the total $\mathrm{N}_{2} \mathrm{O}$ emissions.

- Changes in stratospheric chemistry from emissions of nitrogen oxides $\left(\mathrm{NO}_{x}\right)$ from sub- and supersonic aircraft. The IPCC (2001) estimate of the $\mathrm{NO}_{x}$ emissions from aircraft is $0.2-0.9 \mathrm{TgN} \mathrm{yr}^{-1}$, or $0.2-2 \%$ of the total anthropogenic $\mathrm{NO}_{x}$ emissions. Since aircraft emit their $\mathrm{NO}_{x}$ mostly in the upper troposphere and lower stratosphere they may have a larger effect on the ozone layer than these percentages suggest. Calculations (IPCC, 1999) show that aircraft emissions have increased $\mathrm{NO}_{x}$ concentrations at cruise altitudes in the northern hemisphere by about $20 \%$ with a corresponding calculated total ozone increase of about $0.4 \%$. This change in ozone column includes the increase in tropospheric ozone by aircraft $\mathrm{NO}_{x}$ emissions.

\section{Conclusions}

The emissions from the various transport sectors cover a broad mix of gases and aerosols with very different characteristics and effects on climate and stratospheric ozone. Tools to compare these emissions and put them on a common scale have a number of uses, e.g. to include these emissions in climate policies. In the assessment of effects of current emissions on climate one needs to choose a time horizon as well as an impact parameter (e.g. forcing, temperature, sea level, damage). The choice of time horizon goes beyond natural sciences and requires value judgements; the perceived relative importance of different emissions can depend greatly on that choice. In the choice of impact parameter - which also cannot be solely a science-based decision - there is a trade-off between relevance and uncertainty. The design of a metric depends also on its application and the policy context within which it is intended to function.

In this assessment we have chosen to focus on the metrics GWP, GTP and ODP that are based on physical parameters, but it is recognised that damage-based metrics using discounting of future effects are useful for analysis and exploring various policy options.

Given that there is a need for metrics to assess the importance of short-lived emissions (but not necessarily to use them in a climate agreement) e.g. from the transport sector, we give an overview of existing estimates for GWPs and ODPs from the literature. In addition, by extracting information on lifetimes, radiative forcing strengths, etc. from various published studies we have also established a comprehensive overview of values for these metrics that are not given explicitly in the literature. These values show the dependence on model, various assumptions, region of emissions etc. and give a useful overview of the current state of knowledge.

GWPs are straightforward to calculate for long-lived gases, but become significantly more problematic and uncertain for shortlived components addressed here; $\mathrm{NO}_{x}, \mathrm{CO}, \mathrm{VOC}, \mathrm{BC}, \mathrm{OC}$ and $\mathrm{SO}_{2}$. For GTPs, there is an additionally set of uncertainties, related to the representation of the climate system (the climate sensitivity and the response time) which affects both short- and long-lived components. There are basically two types of uncertainty; namely structural and parametric uncertainty. The first is related to design of the metric and is dependent on the policy context, while the other depends on the current state of scientific understanding.

For the long-lived greenhouse gases, the scientific confidence in metric values can be regarded as relatively high. For the short-lived components, the scientific confidence is low for $\mathrm{NO}_{x}, \mathrm{BC}, \mathrm{OC}$ and $\mathrm{SO}_{2}$. There are particular difficulties for $\mathrm{NO}_{x}$, because the net impact is a small residual of opposing effects which have quite different spatial distributions. The gases $\mathrm{CO}$ and VOC fall within an intermediate category.

The GWP and GTP metrics represent two fundamentally different ways of comparing emissions. While GWP integrates the radiative forcing along the time path up to the chosen time horizon, and puts equal weight on all times between the emission and the time horizon, the GTP focuses on one particular chosen point in time and gives the temperature effect at that time. For short-lived gases this difference in metric design has a large effect on the metric values since the climate system has only a limited memory of the signal of the short-lived emissions after approximately a decade. Thus, the choice of metric is dependent on the perspective adopted in climate policies. The formulation of the EU climate target of $2{ }^{\circ} \mathrm{C}$ gives signals to the scientific community regarding adequate design of metrics and their potential application. As stated by, for example, Manne and Richels (2001), the GWP is not suited for this type of policy. A timevariant metric which takes into account the proximity to the target is more adequate in this situation. This is one of the main reasons why the GTP metric is included in this assessment. As shown in Section 5.4, this metric is also suitable for evaluating the effect of emissions scenarios, and not only emissions for one year.

Our assessment and overview of metric values shows a large spread in the values for $\mathrm{NO}_{x}$ because of the spread in the output from the complex models (due to either underlying scientific uncertainty and/or differences in experimental design). This spread should be acknowledged in any application which uses these values. The region of emissions (both horizontally and vertically) has a large effect on the values, and in addition, the results also indicate large model dependence. In general, low latitude emissions cause larger forcings through changes in ozone and methane. The net effect of three different responses (short-lived ozone, methane and methane-induced ozone) shows large variations. For the aerosols there are several studies giving information for estimating GWPs and GTPs from emissions in different regions, but the results do not give a consistent picture regarding the most and least sensitive regions. Thus we focus mainly on the global-mean values for BC, OC and sulphate. We also give GWP and GTP values for the shipping sector (for $\mathrm{NO}_{x}$ and sulphate) and for aviation $\left(\mathrm{NO}_{x}\right)$.

Improvement of the quality of the metrics and their values will depend on progress in two different areas. Regarding the scientific knowledge and parametric uncertainty, improvements can be expected as more studies and coordinated exercises are published. Regarding the structural uncertainty, choices cannot be made by the natural sciences alone; significant progress requires interaction with economists and researchers from other disciplines, as well as with policymakers regarding the applications and policy contexts. In dialogue with policymakers, we can provide metrics that meet their needs based on the current level of scientific understanding.

\section{Acknowledgements}

This assessment has been funded by the EU FP6 Specific Support Action ATTICA (European Assessment of Transport Impacts on Climate Change and Ozone Depletion) and supported by the EU FP6 Integrated Project QUANTIFY. We thank all ATTICA co-authors, Olivier Boucher and the reviewers, Daniel Johansson and Piers Forster, for their helpful comments on the manuscript. Robert Sausen and Gabriele Erhardt are thanked for their work in coordinating ATTICA. Daniella Iachetti and Tone Veiby provided editorial help.

\section{Appendix 1. The Radiative Forcing Index (RFI)}

The issues concerning the use of the RFI deserve particular comment. The RFI is defined as "the ratio of total radiative forcing 
to that from $\mathrm{CO}_{2}$ emissions alone" (IPCC, 1999). The RFI may be a useful index for indicating the contribution of the non- $\mathrm{CO}_{2}$ climate effects of a given sector or sectors. However, the RFI is being used by some carbon offset companies, and has been proposed to be used in some policymaking applications as a simple multiplier to include the impact of non- $\mathrm{CO}_{2}$ emissions from aviation.

Quite apart from the difficulties in defining an appropriate value for the RFI, the application itself is flawed, as discussed in Wit et al. (2005) and Forster et al. (2006), because it is not an emissions metric. As illustrated in Section 6.1, present-day $\Delta F$ is affected by emissions from many different time periods in the past; this is why radiative forcing and associated measures such as the RFI should not be used to assess the impact of present emissions on future climate. The radiative forcing at the present time is the result of all prior aircraft emissions. In the case of $\mathrm{CO}_{2}$, these emissions will continue to cause a forcing for many decades into the future. By contrast, for the short-lived emissions, the forcing due to these prior emissions will die away rapidly. The RFI does not take this into account as it essentially captures the maximum effect of the short-lived emissions, but fails to account for the persistence of $\mathrm{CO}_{2}$ and thus underplays its climate impact.

As an illustration of this, consider a hypothetical case where current emissions are kept constant indefinitely into the future. $\Delta F$ from $\mathrm{CO}_{2}$ will continue to increase, whereas the other $\Delta F$ s will remain more-orless constant because of their much shorter lifetime. Thus, the RFI decreases over time (see Fig. A1) and the current RFI substantially overestimates the role of non- $\mathrm{CO}_{2}$ effects at, say, the 100 -year time horizon of the Kyoto protocol. This illustrates that this particular metric (and, indeed, radiative forcing itself) is strongly dependent on the particular past history (or future scenario) of emissions; arguably, metrics with such strong dependence should be avoided.

A further concern is that the use of RFI might encourage inappropriate measures. Since the RFI, in current usage, represents a fixed "tax" on non- $\mathrm{CO}_{2}$ emissions, there is no immediate incentive to reduce these non- $\mathrm{CO}_{2}$ emissions - indeed it may appear beneficial to change operations or engine characteristics to increase these emissions where they lead to lower $\mathrm{CO}_{2}$ emissions. Or if the value of RFI was subject to revision, its use might suggest that it is

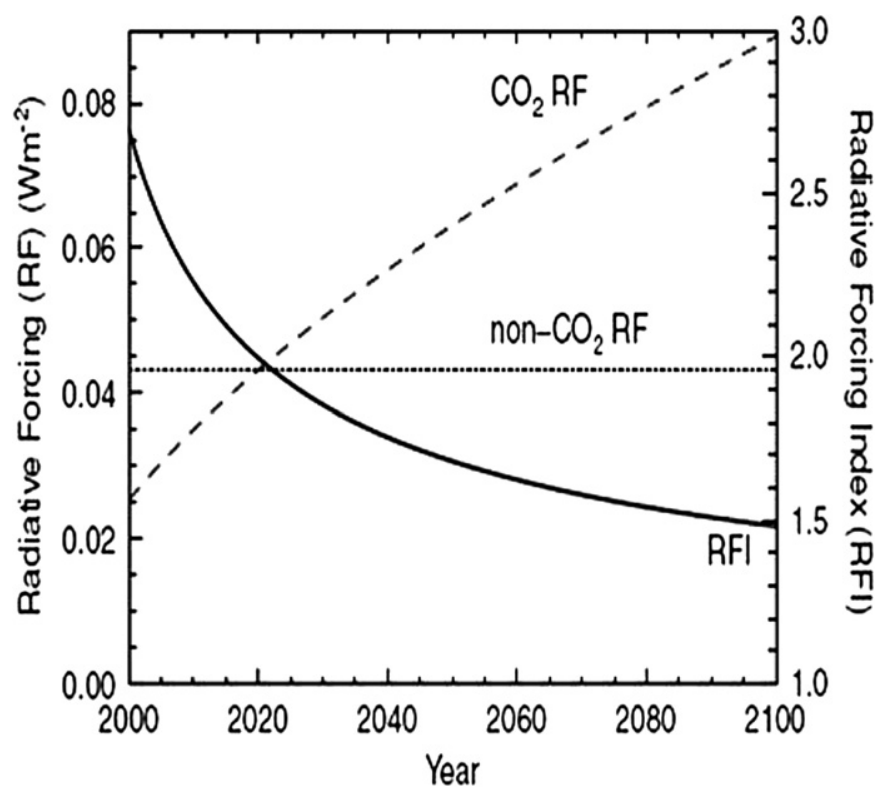

Fig. A1. A scenario for sustained present-day emissions illustrating how $\mathrm{CO}_{2}$ and its $\Delta F$ (dashed line) will continue to increase, whereas the non- $\mathrm{CO}_{2}$ effects (dotted line) have roughly stabilised with the emissions and are not expected to change. As a consequence of this the RFI (solid line) does not remain constant, but decreases over time (from Forster et al., 2006). beneficial (since it would reduce the RFI) to reduce contrail formation by flying lower at the cost of using more fuel and emitting more $\mathrm{CO}_{2}$; however, in reality, the long-lived $\mathrm{CO}_{2}$ emission are likely to have the greater climate impact over time.

An additional consideration is that methodologies adopted for one sector ought to be adopted in a similar way by all sectors. Suggestions for application of the RFI appear to have been limited uniquely to the aviation sector. Recent results (Fuglestvedt et al., 2008; Lauer et al., 2007; Lee et al., 2006) indicate that for shipping, for example, the RFI is negative, because the large cooling influence due to sulphur and $\mathrm{NO}_{x}$ emissions overwhelms the warming effect of $\mathrm{CO}_{2}$. The implications of adopting such a negative RFI, for both climate and air quality policy, have not been addressed, but would appear severe. The logical conclusion of using the RFI as a multiplier in an emission trading scheme would appear to be that shipping would be granted carbon credits for having a negative RFI.

More generally, the GWP has been adopted as the primary means of comparing the climate effect of emissions within the Kyoto Protocol. If the impact of non- $\mathrm{CO}_{2}$ emissions of aviation is to be included in any emission trading schemes, a GWP framework would appear to be more consistent; it is certainly the case that there are no greater difficulties in defining aviation GWPs than there are in defining aviation RFIs.

\section{Appendix 2. Analytical expressions for the Global Temperature Change Potential}

The simplest form of the GTP is given by Equation (7). We use, instead, the approach of Boucher and Reddy (2008) (see their appendix A), where GCM output is used to derive an impulseresponse function which includes the thermal inertia associated with the deep ocean. Since that paper does not present the resulting analytical expressions for the GTP, we do so here. Table A1 presents the parameters used here.

The time-evolution of the radiative forcing due to a pulse emission of $\mathrm{CO}_{2}$ is given by

$\Delta F^{\mathrm{CO}_{2}}(t)=A^{\mathrm{CO}_{2}}\left(a_{0}+\sum_{i=1}^{3} a_{i} \exp \left(\frac{-t}{\alpha_{i}}\right)\right)$

where $A^{\mathrm{CO}_{2}}$ is the specific forcing due to $\mathrm{CO}_{2}$ taken here to be $1.82 \times 10^{-15} \mathrm{Wm}^{-2} \mathrm{~kg}^{-1}$ following Forster et al. (2007a) and the term in parentheses is the decay of a pulse emission of $\mathrm{CO}_{2}$ with time $t$, as adopted for the calculation of the GWP in the same work. Table A1 gives the values for $a_{i}$ and $\alpha_{i}$.

The response in global-mean surface temperature to a unit pulse of $\Delta F$ is taken from Boucher and Reddy (2008) and is given by

$R(t)=\sum_{j=1}^{2} \frac{c_{j}}{d_{j}} \exp \left(-\frac{t}{d_{j}}\right)$

where the parameters $c_{i}$ and $d_{i}$ are given in Table A1. The first term in the summation can crudely be associated with the response of the ocean mixed-layer to a forcing, the second term as the response of the deep ocean, although they are derived from a curve fit to the GCM results and such a physical interpretation is only tentative.

Table A1

Parameter values for the pulse-response function for concentration of $\mathrm{CO}_{2}$ and response to a pulse of radiative forcing used in the AGTP calculations.

\begin{tabular}{llccl}
\hline & 0 & 1 & 2 & 3 \\
\hline$a_{i}$ (unitless) & 0.217 & 0.259 & 0.338 & 0.186 \\
$\alpha_{i}$ (years) & & 172.9 & 18.51 & 1.186 \\
$\left.c_{j}\left(\mathrm{~K}^{-2} \mathrm{Wm}^{-2}\right)^{-1}\right)$ & & 0.631 & 0.429 & \\
$d_{j}$ (years) & & 8.4 & 409.5 & \\
\hline
\end{tabular}


The equilibrium climate sensitivity is given by the sum of the $c_{i}$ values - in this case, $1.06 \mathrm{~K}\left(\mathrm{Wm}^{-2}\right)^{-1}$ equivalent to a $3.9 \mathrm{~K}$ equilibrium response to a doubling of $\mathrm{CO}_{2}$. The AGTP can then be found by analytical integration of the expression given in Section 5.4

$\operatorname{AGTP}(H)=\int_{0}^{H} \Delta F(t) R(H-t) \mathrm{d} t$

where the response to a pulse in radiative forcing $R(H-t)$ is given by Equation (A2).

Using Equations (A1) and (A2), the AGTP for $\mathrm{CO}_{2}$ for a time horizon $H$ is given by

$$
\begin{aligned}
\operatorname{AGTP}^{\mathrm{CO}_{2}}(H)= & A^{\mathrm{CO}_{2}}\left\{\sum_{j=1}^{2} a_{0} c_{j}\left(1-\exp \left(-\frac{H}{d_{j}}\right)\right)\right. \\
& \left.+\sum_{i=1}^{3} \sum_{j=1}^{2} \frac{a_{i} \alpha_{i} c_{j}}{\alpha_{i}-d_{j}}\left(\exp \left(-\frac{H}{\alpha_{j}}\right)-\exp \left(-\frac{H}{d_{j}}\right)\right)\right\} .
\end{aligned}
$$

The AGTP for non- $\mathrm{CO}_{2}$ greenhouse gases, aerosols, contrails and stratospheric water vapour is simpler to compute (and similar in form to Equation (8)), as we assume that concentration (and hence $\Delta F$ ) following a pulse emission is a simple exponential decay:

$\operatorname{AGTP}_{x}(H)=\sum_{j=1}^{2} \frac{A^{x} \alpha c_{j}}{\left(\alpha-d_{j}\right)}\left(\exp \left(\frac{-H}{\alpha}\right)-\exp \left(\frac{-H}{d_{j}}\right)\right)$

where $A^{x}$ and $\alpha$ are the specific radiative forcing and lifetime, respectively.

For species such as $\mathrm{NO}_{x}$ which lead to changes in ozone and methane, as explained in Section 7.2, the forcing is assumed to be a result of a one-year step emission of the pre-cursors in year 1 followed by an exponential decay of the resulting forcing from the end-of-year 1 value after this.

First it is necessary to derive the steady-state $\Delta F_{\mathrm{O}_{3}}^{\mathrm{SS}}$ corresponding to the integrated radiative forcings reported in the studies that use pulses. We assume that the integrated RF of a pulse emission and a one-year step emissions are equal. This assumption is valid if we assume that same number of ozone molecules is produced in the pulse case and in the one-year step emission case, and that their lifetimes are equal.

The forcing history from a pulse is given by $\Delta F_{\mathrm{O}_{3}}^{p}(t)$, and the corresponding forcing from a one-year step emission is given by $\Delta F_{\mathrm{O} 3}^{S}(t)$. It follows from the assumption above that

$\int_{0}^{H} \Delta F_{\mathrm{O}_{3}}^{p}(t) \mathrm{d} t=\int_{0}^{H} \Delta F_{\mathrm{O}_{3}}^{S}(t) \mathrm{d} t$ for $H \gg a$

The left side can be written as

$$
\int_{0}^{H} \Delta F_{\mathrm{O}_{3}}^{p}(t) \mathrm{d} t=\int_{0}^{H} \Delta F_{0} \exp \left(-t / \alpha_{s}\right) \mathrm{d} t .
$$

And the right hand side can be written as

$$
\begin{aligned}
\int_{0}^{H} \Delta F_{\mathrm{O}_{3}}^{S}(t) \mathrm{d} t= & \int_{0}^{1} \Delta F_{\mathrm{O}_{3}}^{S, S \mathrm{~S}}\left(1-\exp \left(-t / \alpha_{S}\right)\right) \mathrm{d} t \\
& +\int_{1}^{H} \Delta F_{\mathrm{O}_{3}}^{S, S S}\left(1-\exp \left(-1 / \alpha_{S}\right)\right) \exp \left(-(t-1) / \alpha_{S}\right) \mathrm{d} t,
\end{aligned}
$$

where the first term integrates the effects over the first year (when emission occurs) and the next term integrates the long-term effects of the decay of the first year effect,

When the lifetime $\left(\alpha_{S}\right)$ is short compared to the time horizon $(H)$ it is readily shown that the integrated forcing in the above equation is equal to $\Delta F^{S S} \cdot 1$ year.

Once the $\Delta F$ is defined, Equations (10)-(14) can be used in conjunction with Equation (A3) to derive the components of the AGTP. This leads to the following expressions (which are valid provided that $H>1$ year) so that the total AGTP is then the sum of (A5) and (A9):

a. Short-lived $\mathrm{O}_{3}$ perturbation $(t<1)$

For the forcing given by Equation (10), the AGTP is

$$
\begin{gathered}
\operatorname{AGTP}_{\mathrm{O}_{3}}^{S}(H)=\Delta F_{\mathrm{O}_{3}}^{S, S S} \sum_{j=1}^{2}\left\{c_{j}\left(\exp \left(\frac{1-H}{d_{j}}\right)-\exp \left(-\frac{H}{d_{j}}\right)\right)+\frac{c_{j} \alpha_{s}}{\alpha_{s}-d_{j}}\left(\exp \left(-\frac{H}{d_{j}}\right)\right.\right. \\
\left.\left.-\exp \left(\frac{1-H}{d_{j}}\right) \exp \left(-\frac{1}{\alpha_{s}}\right)\right)\right\}
\end{gathered}
$$

b. Short-lived $\mathrm{O}_{3}$ perturbation $(t \geq 1)$

For the forcing given by Equation (11), the AGTP is

$$
\begin{aligned}
\operatorname{AGTP}_{\mathrm{O}_{3}}^{S}(H)= & \Delta F_{\mathrm{O}_{3}}^{S, S S}\left(1-\exp \left(-\frac{1}{\alpha_{S}}\right)\right) \sum_{j=1}^{2} \frac{\alpha_{s} c_{j}}{\alpha_{S}-d_{j}}\left(\exp \left(\frac{1-H}{\alpha_{S}}\right)\right. \\
& \left.-\exp \left(\frac{1-H}{d_{j}}\right)\right)
\end{aligned}
$$

c. Methane perturbation $(t<1)$

For the forcing given by Equation (12), the AGTP is

$$
\begin{aligned}
\operatorname{AGTP}_{\mathrm{CH}_{4}}(H)= & \Delta F_{\mathrm{CH}_{4}}^{\mathrm{SS}} \sum_{j=1}^{2}\left\{c _ { j } \left(\exp \left(\frac{1-H}{d_{j}}\right)\right.\right. \\
& \left.-\exp \left(-\frac{H}{d_{j}}\right)\right)+\frac{c_{j} \alpha_{P M}}{\alpha_{P M}-d_{j}} \\
\times & \left.\left(\exp \left(-\frac{H}{d_{j}}\right)-\exp \left(\frac{1-H}{d_{j}}\right) \exp \left(-\frac{1}{\alpha_{P M}}\right)\right)\right\}
\end{aligned}
$$

d. Methane perturbation $(t \geq 1)$

For the forcing given by Equation (13) the AGTP is

$$
\begin{aligned}
\operatorname{AGTP}_{\mathrm{CH}_{4}}(H)= & \Delta F_{\mathrm{CH}_{4}}^{\mathrm{SS}}\left(1-\exp \left(-\frac{1}{\alpha_{P M}}\right)\right) \\
& \times \sum_{j=1}^{2} \frac{\alpha_{P M} c_{j}}{\alpha_{P M}-d_{j}}\left(\exp \left(\frac{1-H}{\alpha_{P M}}\right)-\exp \left(\frac{1-H}{d_{j}}\right)\right)
\end{aligned}
$$

e. Methane-induced $\mathrm{O}_{3}$ perturbation $(t \geq 1)$

For the forcing given by Equation (14) the AGTP is

$$
\begin{aligned}
\operatorname{AGTP}_{\mathrm{O}_{3}}^{P M}(H)= & \Delta F_{\mathrm{O}_{3}}^{P M, S S}\left(1-\exp \left(-\frac{1}{\alpha_{P M}}\right)\right) \\
& \times \sum_{j=1}^{2} \frac{\alpha_{P M} c_{j}}{\alpha_{P M}-d_{j}}\left(\exp \left(\frac{1-H}{\alpha_{P M}}\right)-\exp \left(\frac{1-H}{d_{j}}\right)\right)
\end{aligned}
$$

The AGWP for the short-lived ozone forcing given by Equations (10) and (11) is 


$$
\begin{aligned}
\operatorname{AGWP}_{\mathrm{O}_{3}}^{S}(H)= & \Delta F_{\mathrm{O}_{3}}^{S, S S}\left(1-\alpha_{S}\left(\exp \left(-\frac{(H-1)}{\alpha_{S}}\right)\right.\right. \\
& \left.\left.-\exp \left(-\frac{H}{\alpha_{S}}\right)\right)\right)
\end{aligned}
$$

with a similar expression for the methane forcings. The methane-induced ozone forcing follows from using Equation (14) for the pulse forcing in the calculation of the GWP.

\section{Appendix 3. Regional value of aerosol metrics}

In this Appendix we illustrate the regional variation of emissions metrics based on four recent studies (Berntsen et al., 2006; Koch et al., 2007; Naik et al., 2007; Reddy and Boucher, 2007). In all studies, the forcing resulting from emissions from distinct regions were calculated, and are used here to compute the metrics. It is emphasized that these studies adopted different experimental design and, in particular, Naik et al. (2007) focused on biomass emissions, rather than transport emissions; since these emissions have a different seasonality to transport emissions, their results are probably not directly comparable.

Tables A2-A4 present the GWP and GTP results for black carbon, organic carbon and sulphate respectively, and include the global multi-model average results from Schulz et al. (2006) (see Table 5) for comparison. We retain the geographical designations given in

\section{Table A2}

GWP values for one-year pulse emissions of black carbon for a 20, 100 and 500 year time horizons and GTP values for 20,50 and 100 years. The effects of aerosols on clouds (and in the case of black carbon, on surface albedo) are not included. The GTP

\begin{tabular}{|c|c|c|c|c|c|c|}
\hline \multirow[t]{2}{*}{ Study } & \multicolumn{3}{|l|}{ GWP } & \multicolumn{3}{|l|}{ GTP } \\
\hline & $H=20$ & $H=100$ & $H=500$ & $H=20$ & $H=50$ & $H=100$ \\
\hline \multicolumn{7}{|l|}{ Koch et al. } \\
\hline SE ASIA & 1700 & 480 & 150 & 500 & 82 & 68 \\
\hline N AMER & 1900 & 550 & 170 & 560 & 93 & 77 \\
\hline EURO & 1800 & 510 & 150 & 520 & 86 & 72 \\
\hline S ASIA & 3200 & 920 & 280 & 940 & 160 & 130 \\
\hline S AMER & 2200 & 610 & 190 & 630 & 100 & 87 \\
\hline AFRICA & 1200 & 340 & 100 & 350 & 57 & 48 \\
\hline \multicolumn{7}{|l|}{ Naik et al. } \\
\hline Africa & 4500 & 1300 & 390 & 1300 & 220 & 180 \\
\hline E ASIA & 3400 & 960 & 290 & 980 & 160 & 140 \\
\hline FSU & 2000 & 580 & 180 & 590 & 98 & 81 \\
\hline INDIA & 5100 & 1400 & 440 & 1500 & 250 & 200 \\
\hline N AMER & 3200 & 920 & 280 & 940 & 160 & 130 \\
\hline S AMER & 4900 & 1400 & 420 & 1400 & 240 & 200 \\
\hline SE ASIA & 4000 & 1200 & 350 & 1200 & 200 & 160 \\
\hline \multicolumn{7}{|c|}{ Reddy and Boucher } \\
\hline S. America & 1900 & 550 & 170 & 570 & 94 & 78 \\
\hline N AMER & 1500 & 430 & 130 & 450 & 74 & 62 \\
\hline AFRICA & 2500 & 720 & 220 & 730 & 120 & 100 \\
\hline EUR & 1400 & 380 & 120 & 390 & 65 & 54 \\
\hline Mid-EAST & 2600 & 740 & 220 & 760 & 130 & 100 \\
\hline S ASIA & 2400 & 670 & 200 & 690 & 110 & 95 \\
\hline E ASIA & 1500 & 420 & 130 & 430 & 72 & 60 \\
\hline \multicolumn{7}{|l|}{ Berntsen et al. } \\
\hline EUR & 1500 & 430 & 130 & 440 & 72 & 60 \\
\hline China & 1200 & 340 & 100 & 350 & 58 & 48 \\
\hline S. ASIA & 2200 & 640 & 190 & 660 & 110 & 91 \\
\hline S AMER & 2200 & 620 & 190 & 634 & 110 & 88 \\
\hline Bond and Sun & 2200 & 680 & & & & \\
\hline \multicolumn{7}{|l|}{ Schulz et al. } \\
\hline Global mean & 1600 & 460 & 140 & 470 & 77 & 64 \\
\hline
\end{tabular}
values are specific to a given value of climate sensitivity - see Appendix 2.
Table A3

GWP values for one-year pulse emissions of organic carbon aerosols for a 20,100 and 500 year time horizons and GTP values for 20, 50 and 100 years. The effect of aerosols on clouds is not included. The GTP values are specific to a given value of

\begin{tabular}{|c|c|c|c|c|c|c|}
\hline \multirow[t]{2}{*}{ Study } & \multicolumn{3}{|l|}{ GWP } & \multicolumn{3}{|l|}{ GTP } \\
\hline & $H=20$ & $H=100$ & $H=500$ & $H=20$ & $H=50$ & $H=100$ \\
\hline \multicolumn{7}{|l|}{ Koch et al. } \\
\hline SE ASIA & -80 & -23 & -7 & -23 & -4 & -3 \\
\hline N AMER & -150 & -42 & -13 & -43 & -7 & -6 \\
\hline EURO & 160 & 45 & 14 & 46 & 8 & 6 \\
\hline S ASIA & -340 & -95 & -29 & -98 & -16 & -13 \\
\hline S AMER & -277 & -79 & -24 & -81 & -13 & -11 \\
\hline AFRICA & -190 & -53 & -16 & -54 & -9 & -7 \\
\hline \multicolumn{7}{|l|}{ Naik et al. } \\
\hline Africa & -470 & -130 & -41 & -140 & -23 & -19 \\
\hline E ASIA & -300 & -86 & -26 & -88 & -15 & -12 \\
\hline FSU & -270 & -77 & -23 & -79 & -13 & -11 \\
\hline INDIA & -510 & -140 & -44 & -150 & -24 & -20 \\
\hline N AMER & -310 & -88 & -27 & -90 & -15 & -12 \\
\hline S AMER & -510 & -150 & -44 & -150 & -25 & -21 \\
\hline SE ASIA & -540 & -150 & -47 & -160 & -26 & -22 \\
\hline \multicolumn{7}{|l|}{ Berntsen et al. } \\
\hline EUR & -170 & -48 & -14 & -49 & -8 & -7 \\
\hline China & -130 & -37 & -11 & -38 & -6 & -5 \\
\hline S. ASIA & -300 & -84 & -26 & -86 & -14 & -12 \\
\hline S AMER & -220 & -62 & -19 & -63 & -10 & -9 \\
\hline \multicolumn{7}{|l|}{ Schulz et al. } \\
\hline Global mean & -240 & -69 & -21 & -71 & -12 & -10 \\
\hline
\end{tabular}
climate sensitivity - see Appendix 2.

the original studies, although these can be ambiguous, particularly when describing Asian emissions. Thus, for Koch et al. (2007) "SE Asia" corresponds, broadly, to China and Siberia and "S Asia" corresponds, broadly, to the Indian sub-continent; for Naik et al. (2007) "E Asia" corresponds broadly to China and "SE Asia" corresponds broadly to a region including Vietnam and Indonesia; in Reddy and Boucher (2007), "S Asia" is broadly the Indian subcontinent, while "E Asia" is broadly Siberia and China. For Berntsen et al. (2006) "S Asia" also corresponds broadly to the Indian subcontinent. For black carbon, we also include the GWP presented by Bond and Sun (2005).

Two important points emerge from these tables. First, when considering individual studies there is a quite substantial variation

Table A4

GWP values for aerosols formed from a one-year pulse emissions of $\mathrm{SO}_{2}$ for a 20, 100 and 500 year time horizons and GTP values for 20,50 and 100 years. The indirect effects of sulphate on clouds are not included. (All values are on $\mathrm{SO}_{2}$ basis. Multiply by 2 to convert from $\mathrm{SO}_{2}$ to $\mathrm{S}$ basis.) The GTP values are specific to a given value of

\begin{tabular}{|c|c|c|c|c|c|c|}
\hline \multirow[t]{2}{*}{ Study } & \multicolumn{3}{|l|}{ GWP } & \multicolumn{3}{|l|}{ GTP } \\
\hline & $H=20$ & $H=100$ & $H=500$ & $H=20$ & $H=50$ & $H=100$ \\
\hline \multicolumn{7}{|l|}{ Koch } \\
\hline SE ASIA & -57 & -16 & -4.9 & -17 & -3 & -2 \\
\hline N AMER & -89 & -25 & -7.6 & -26 & -4 & -4 \\
\hline EURO & -42 & -12 & -3.6 & -12 & -2 & -2 \\
\hline S ASIA & -120 & -35 & -11 & -36 & -6 & -5 \\
\hline S AMER & -570 & -160 & -49 & -170 & -27 & -23 \\
\hline AFRICA & -140 & -38 & -12 & -39 & -7 & -5 \\
\hline \multicolumn{7}{|l|}{ Berntsen et al. } \\
\hline EUR & -100 & -29 & -8.7 & -29 & -5 & -4 \\
\hline China & -69 & -20 & -6.0 & -20 & -3 & -3 \\
\hline S. ASIA & -160 & -44 & -14 & -46 & -8 & -6 \\
\hline S. AMER & -180 & -50 & -15 & -51 & -8 & -7 \\
\hline \multicolumn{7}{|l|}{ Schulz et al. } \\
\hline Global mean & -140 & -40 & -12 & -41 & -7 & -6 \\
\hline
\end{tabular}
climate sensitivity - see Appendix 2. 
in metric values for aerosols (or their pre-cursors) emitted in different regions, typically of a factor of 2 or 3 , but sometimes reaching an order of magnitude for the sulphate case and varying in sign for the organic carbon case. Second, when comparing across studies, there are quite distinct differences in the ranking of the relative importance of regions. As an example, comparing the GWP(20) for black carbon, emissions from Africa are least important in the Koch et al. (2007) calculations, but the value is double (and is the second most important) using the parameters given in Reddy and Boucher (2007).

Hence, it is concluded that the importance of regional variation in emission metrics due to aerosols has been established by these studies, but as yet it is not possible to recommend values for that effect. A tight multi-model intercomparison, along the lines of Schulz et al. (2006), but focusing on regionally constrained emissions, is likely to be required for further progress to be made.

\section{Appendix 4. Major acronyms and abbreviations used in this assessment}

\section{AGWP Absolute Global Warming Potential}

AGTP Absolute Global Temperature Change Potential

AIC Aviation-Induced Cirrus

ATTICA Assessment of Transport Impacts on Climate Change and Ozone Depletion

BC Black Carbon

CCM Chemistry-Climate Model

CICERO Centre for International Climate and Environmental Research (Norway)

CTM Chemical Transport Model

DLR Institut für Physik der Atmosphäre, Oberpfaffenhofen (Germany)

EC European Commission

EECl Effective Equivalent Chlorine

EESC Equivalent Effective Stratospheric Chlorine

EU European Union

FP6 Sixth Framework Programme

GCM General Circulation Model

GHG Greenhouse Gas

GTP Global Temperature Change Potential

GWP Global Warming Potential

IPCC Intergovernmental Panel on Climate Change

LLGHG Long-Lived Greenhouse Gas

LMDz Laboratoire de Météorologie Dynamique

OC Organic Carbon

ODP Ozone Depleting Potential

ODS Ozone Depleting Substance

POA Primary Organic Aerosol

QUANTIFY Quantifying the Climate Impact of Global and European Transport System

$\mathrm{RF} \quad$ Radiative Forcing

RFI Radiative Forcing Index

SCM Simple Climate Model

SOA Secondary Organic Aerosol

TAC International Conference on Transport, Atmosphere and Climate

3-D three-dimensional

UD-EBM Upwelling-Diffusion Energy Balance Model

UiO University of Oslo

UNEP United Nations Environment Programme

UNFCCC United Nations Framework Convention on Climate Change

USEPA United States Environmental Protection Agency

WMO World Meteorological Organization
Appendix 5. Some chemical symbols used in this assessment

\begin{tabular}{|c|c|}
\hline $\mathrm{CCl}_{4}$ & carbon tetrachloride \\
\hline CFC & chlorofluorocarbon \\
\hline CFC-11 & trichlorofluoromethane \\
\hline CFC-12 & dichlorodifluoromethane \\
\hline CFC-113 & trichlorotrifluoroethane \\
\hline CFC-114 & dichlorotetrafluoroethane \\
\hline CFC-115 & chloropentafluoroethane \\
\hline $\mathrm{CH}_{4}$ & methane \\
\hline $\mathrm{CH}_{3} \mathrm{Br}$ & methylbromide \\
\hline $\mathrm{CH}_{3} \mathrm{Cl}$ & methylchloride \\
\hline $\mathrm{CH}_{3} \mathrm{CCl}_{3}$ & methyl chloroform \\
\hline $\mathrm{CO}$ & carbon monoxide \\
\hline $\mathrm{CO}_{2}$ & carbon dioxide \\
\hline $\mathrm{H}_{2}$ & hydrogen molecule \\
\hline halon-1202 & $\mathrm{CBr}_{2} \mathrm{~F}_{2}$ \\
\hline halon-1211 & $\mathrm{CF}_{2} \mathrm{ClBr}$ \\
\hline halon-1301 & $\mathrm{CF}_{3} \mathrm{Br}$ \\
\hline halon-2402 & $\mathrm{CF}_{2} \mathrm{Br} \mathrm{CF} 2 \mathrm{Br}$ \\
\hline $\mathrm{HCFC}$ & hydrochlorofluorocarbon \\
\hline HCFC-22 & $\mathrm{CHF}_{2} \mathrm{Cl}$ \\
\hline HCFC-123 & $\mathrm{C}_{2} \mathrm{~F}_{3} \mathrm{HCl}_{2}$ \\
\hline HCFC-124 & $\mathrm{CF}_{3} \mathrm{CHClF}$ \\
\hline HCFC-141b & $\mathrm{CH}_{3} \mathrm{CFCl}_{2}$ \\
\hline HCFC-142b & $\mathrm{CH}_{3} \mathrm{CF}_{2} \mathrm{Cl}$ \\
\hline HCFC-225ca & $\mathrm{CF}_{3} \mathrm{CF}_{2} \mathrm{CHCl}_{2}$ \\
\hline HCFC-225cb & $\mathrm{CClF}_{2} \mathrm{CF}_{2} \mathrm{CHClF}$ \\
\hline $\mathrm{HFC}$ & hydrofluorocarbon \\
\hline HFC-134a & $\mathrm{CF}_{3} \mathrm{CH}_{2} \mathrm{~F}$ \\
\hline $\mathrm{HO}_{x}$ & the sum of $\mathrm{OH}$ and $\mathrm{HO}_{2}$ \\
\hline $\mathrm{H}_{2} \mathrm{O}$ & water vapour \\
\hline $\mathrm{N}$ & atomic nitrogen \\
\hline NMVOC & non methane volatile organic compounds \\
\hline $\mathrm{NO}_{x}$ & nitrogen oxides $\left(\mathrm{NO}+\mathrm{NO}_{2}\right)$ \\
\hline $\mathrm{NO}_{3}$ & nitrate radical \\
\hline $\mathrm{N}_{2} \mathrm{O}$ & nitrous oxide \\
\hline $\mathrm{O}_{3}$ & ozone \\
\hline $\mathrm{OH}$ & hydroxyl radical \\
\hline PFC & perfluorocarbon \\
\hline $\mathrm{RO}_{x}$ & $\begin{array}{l}\text { organic peroxy radicals }\left(\mathrm{RO}_{2} \text { with } \mathrm{R} \text { being an organic }\right. \\
\text { group) }\end{array}$ \\
\hline S & atomic sulphur \\
\hline $\mathrm{SO}_{2}$ & sulphur dioxide \\
\hline $\mathrm{SO}_{4}^{2-}$ & sulphate ion \\
\hline VOC & volatile organic compounds \\
\hline
\end{tabular}

\section{References}

Aaheim, H.A., Fuglestvedt, J.S., Godal, O., 2006. Cost savings of a flexible multigas climate policy. Energy Journal, 485-502 (special issue).

Archer, D., Kheshgi, H., MaierReimer, E., 1997. Multiple timescales for neutralization of fossil fuel $\mathrm{CO}_{2}$. Geophysical Research Letters 24, 405-408.

Berntsen, T.K., Fuglestvedt, J.S., Joshi, M.M., Shine, K.P., Stuber, N., Ponater, M., Sausen, R., Hauglustaine, D.A., Li, L., 2005. Climate response to regional emissions of ozone precursors: sensitivities and warming potentials. Tellus 57B, 283-304.

Berntsen, T., Fuglestvedt, J., Myhre, G., Stordal, F., Berglen, T.F., 2006. Abatement of greenhouse gases: does location matter? Climatic Change 74, 377-411.

Berntsen, T., Fuglestvedt, J., 2008. Global temperature responses to current emissions from the transport sector. Proceedings of the National Academy of Sciences of the United States of America 105, 19154-19159.

Boer, G.J., Yu, B., 2003. Climate sensitivity and response. Climate Dynamics 21, 415-429.

Bond, T.C., Sun, H.L., 2005. Can reducing black carbon emissions counteract global warming? Environmental Science \& Technology 39, 5921-5926.

Boucher, O., Reddy, M.S., 2008. Climate trade-off between black carbon and carbon dioxide emissions. Energy Policy 36, 193-200. 
Bradford, D.F., 2001. Time, money and tradeoffs. Nature 410, 649-650.

Caldeira, K., Kasting, J.F., 1993. Insensitivity of global warming potentials to carbon dioxide emission scenarios. Nature 366, 251-253.

Capaldo, K., Corbett, J.J., Kasibhatla, P., Fischbeck, P.S., Pandis, S.N., 1999. Effects of ship emissions on sulphur cycling and radiative climate forcing over the ocean. Nature 400, 743-746.

Collins, W.J., Derwent, R.G., Johnson, C.E., Stevenson, D.S., 2002. The oxidation of organic compounds in the troposphere and their global warming potentials. Climatic Change 52, 453-479.

Daniel, J.S., Solomon, S., Albritton, D.L., 1995. On the evaluation of halocarbon radiative forcing and global warming potentials. Journal of Geophysical Research-Atmospheres 100, 1271-1286.

den Elzen, M., Meinshausen, M., 2006. Meeting the EU 2 degrees $C$ climate target: global and regional emission implications. Climate Policy 6, 545-564.

Derwent, R.G., Collins, W.J., Johnson, C.E., Stevenson, D.S., 2001. Transient behaviour of tropospheric ozone precursors in a global 3-D CTM and their indirect greenhouse effects. Climatic Change 49, 463-487.

Derwent, R.G., Stevenson, D.S., Doherty, R.M., Collins, W.J., Sanderson, M.G., Johnson, C.E., 2008. Radiative forcing from surface $\mathrm{NO}_{x}$ emissions: spatial and seasonal variations. Climatic Change 88, 385-401.

Eckaus, R.S., 1992. Comparing the effects of greenhouse gas emissions on global warming. Energy Journal 13.

Endresen, Ø., Sørgård, E., Sundet, J.K., Dalsøren, S.B., Isaksen, I.S.A., Berglen, T.F. Gravir, G., 2003. Emission from international sea transportation and environmental impact. Journal of Geophysical Research 108, 4560.

Eyring, V., Kohler, H.W., van Aardenne, J., Lauer, A., 2005. Emissions from international shipping: 1 . The last 50 years. Journal of Geophysical Research-Atmospheres 110, D17305.

Eyring, V., Stevenson, D.S., Lauer, A., Dentener, F.J., Butler, T., Collins, W.J., Ellingsen, K., Gauss, M., Hauglustaine, D.A., Isaksen, I.S.A., Lawrence, M.G. Richter, A., Rodriguez, J.M., Sanderson, M., Strahan, S.E., Sudo, K., Szopa, S., van Noije, T.P.C., Wild, O., 2007. Multi-model simulations of the impact of international shipping on atmospheric chemistry and climate in 2000 and 2030. Atmospheric Chemistry and Physics 7, 757-780.

Eyring, V., Isaksen, I.S.A., Berntsen, T., Collins, W.J., Corbett, J.J., Endresen, O., Grainger, R.G., Moldanova, J., Schlager, H., Stevenson, D.S., 2010. Transport impacts on atmosphere and climate: shipping. Atmospheric Environment 44, 4735-4771.

Fisher, D.A., Hales, C.H., Filkin, D.L., Ko, M.K.W., Sze, N.D., Connell, P.S., Wuebbles, D.J., Isaksen, I.S.A., Stordal, F., 1990a. Model calculations of the relative effects of CFCs and their replacements on stratospheric ozone. Nature 344, 508-512.

Fisher, D.A., Hales, C.H., Wang, W.C., Ko, M.K.W., Sze, N.D., 1990b. Model calculations of the relative effects of CFCs and their replacements on global warming. Nature 344, 513-516.

Forster, P.M.D., Shine, K.P., Stuber, N., 2006. It is premature to include non- $\mathrm{CO}_{2}$ effects of aviation in emission trading schemes. Atmospheric Environment 40, 1117-1121.

Forster, P., Ramaswamy, V., Artaxo, P., Berntsen, T., Betts, R., Fahey, D.W., Haywood, J., Lean, J., Lowe, D.C., Myhre, G., Nganga, J., Prinn, R., Raga, G., Schulz, M., Van Dorland, R., 2007a. Changes in atmospheric constituents and in radiative forcing. In: Solomon, S., et al. (Eds.), Climate Change 2007: the Physical Science Basis. Contribution of Working Group I to the Fourth Assessment Report of the Intergovernmental Panel on Climate Change. Cambridge University Press, Cambridge.

Forster, P.M.D., Shine, K.P., Stuber, N., 2007b. Corrigendum to "It is premature to include non- $\mathrm{CO}_{2}$ effects of aviation in emission trading schemes (vol 40, pg 1117, 2006)". Atmospheric Environment 41 3941-3941.

Forster, P., Rogers, H., 2008. Metrics for Comparison of Climate Impacts from Well Mixed Greenhouse Gases and Inhomogeneous Forcing Such as Those from UT/ LS Ozone, Contrails and Contrail Cirrus. (Subject Specific White Paper). Aviation Climate Change Research Initiative (ACCRI). Available from: www.faa.gov/ about/office_org/headquarters_offices/aep/aviation_climate/media/ACCRI SSWP_VII_Forster.pdf.

Freeman, A.M., 2003. The Measurement of Environmental and Resource Values, second ed.. Resources for the Future Press, Washington, D.C.

Fuglestvedt, J.S., Berntsen, T.K., Godal, O., Sausen, R., Shine, K.P., Skodvin, T., 2003. Metrics of climate change: assessing radiative forcing and emission indices. Climatic Change 58, 267-331.

Fuglestvedt, J.S., Berntsen, T.K., Isaksen, I.S.A., Mao, H.T., Liang, X.Z., Wang, W.C., 1999. Climatic forcing of nitrogen oxides through changes in tropospheric ozone and methane; global 3D model studies. Atmospheric Environment 33, 961-977.

Fuglestvedt, J., Berntsen, T., Myhre, G., Rypdal, K., Bieltvedt Skeie, R., 2008. Climate forcing from the transport sectors. Proceedings of the National Academy of Sciences of the United States of America 105, 454-458.

Godal, O., Fuglestvedt, J., 2002. Testing 100-year global warming potentials: impacts on compliance costs and abatement profile. Climatic Change 52, 93-127.

Grewe, V., Stenke, A., 2008. A strategy for climate evaluation of aircraft technology: an efficient climate impact assessment tool - AirClim. Atmospheric Chemistry and Physics 8, 4621-4639.

Hansen, J., Sato, M., Ruedy, R., Nazarenko, L., Lacis, A., Schmidt, G.A., Russell, G., Aleinov, I., Bauer, M., Bauer, S., Bell, N., Cairns, B., Canuto, V., Chandler, M., Cheng, Y., Del Genio, A., Faluvegi, G., Fleming, E., Friend, A., Hall, T., Jackman, C., Kelley, M., Kiang, N., Koch, D., Lean, J., Lerner, J., Lo, K., Menon, S., Miller, R., Minnis, P., Novakov, T., Oinas, V., Perlwitz, J., Perlwitz, J., Rind, D., Romanou, A.,
Shindell, D., Stone, P., Sun, S., Tausnev, N., Thresher, D., Wielicki, B., Wong, T., Yao, M., Zhang, S., 2005. Efficacy of climate forcings. Journal of Geophysical Research-Atmospheres 110, D18104.

Hartmann, D.L., 1996. Global Physical Climatology. Academic.

IPCC, 1990. Climate Change: the Intergovernmental Panel on Climate Change Scientific Assessment. Cambridge University Press, Cambridge, UK.

IPCC, 1992. Climate Change 1992. The Intergovernmental Panel on Climate Change Scientific Assessment. Cambridge University Press, Cambridge, UK.

IPCC, 1995. Climate Change 1994. The Intergovernmental Panel on Climate Change Cambridge University Press, Cambridge, UK.

IPCC, 1996. Climate Change 1995. The Science of Climate Change. The Intergovernmental Panel on Climate Change. Cambridge University Press, Cambridge, UK.

IPCC, 1999. Aviation and the Global Atmosphere. Intergovernmental Panel on Climate Change. Cambridge University Press, Cambridge, UK.

IPCC, 2001. Climate Change 2001: the Scientific Basis. Intergovernmental Panel on Climate Change. Cambridge University Press, Cambridge, UK.

IPCC, 2005. Safeguarding the Ozone Layer and the Global Climate System. Intergovernmental Panel on Climate Change. Cambridge University Press, Cambridge.

IPCC, 2007. Climate Change 2007 - the Physical Science Basis. Contribution of Working Group I to the Fourth Assessment Report of the IPCC. Cambridge University Press, Cambridge.

IPCC, 2009. Summary Report of the IPCC Expert Meeting on the Science of Alternative Metrics 18-20 March 2009, Oslo, Norway. IPCC. IPCC-XXX/Doc.13 (31.III.2009) Available at: www.ipcc.ch/meetings/session30/doc13.pdf.

Johansson, D.J.A., Persson, U.M., Azar, C., 2006. The cost of using global warming potentials: analyzing the trade off between $\mathrm{CO}_{2}, \mathrm{CH}_{4}$ and $\mathrm{N}_{2} \mathrm{O}$. Climatic Change 77, 291-309.

Johnson, C., Henshaw, J., McInnes, G., 1992. Impact of aircraft and surface emissions of nitrogen oxides on tropospheric ozone and global warming. Nature 355, 69-71.

Joos, F., Bruno, M., Fink, R., Siegenthaler, U., Stocker, T.F, LeQuere, C., 1996. An efficient and accurate representation of complex oceanic and biospheric models of anthropogenic carbon uptake. Tellus Series B-Chemical and Physical Meteorology 48, 397-417.

Kahn Ribeiro, S., Kobayashi, S., Beuthe, M., Gasca, J., Greene, D., Lee, D.S. Muromachi, Y., Newton, P.J., Plotkin, S., Sperling, D., Wit, R., Zhou, P.J., 2007. Transport and its infrastructure. In: Metz, B., et al. (Eds.), Climate Change 2007: Mitigation. Contribution of Working Group III to the Fourth Assessment Report of the Intergovernmental Panel on Climate Change. Cambridge University Press, Cambridge.

Koch, D., Bond, T.C., Streets, D., Unger, N., van der Werf, G.R., 2007. Global impacts of aerosols from particular source regions and sectors. Journal of Geophysical Research-Atmospheres 112, D02205.

Köhler, M.O., Rädel, G., Dessens, O., Shine, K.P., Rogers, H.L., Wild, O., Pyle, J.A., 2008. Impact of perturbations to nitrogen oxide emissions from global aviation. Journal of Geophysical Research 113, D11305.

Kolstad, C.D., 2000. Environmental Economics. Oxford University Press, New York

Lauer, A., Eyring, V., Hendricks, J., Jockel, P., Lohmann, U., 2007. Global mode simulations of the impact of ocean-going ships on aerosols, clouds, and the radiation budget. Atmospheric Chemistry and Physics 7, 5061-5079.

Lee, D.S., Lim, L., Eyring, V., Sausen, R., Endresen, O., Behrens, H.-L., 2006. Radiative forcing and temperature response from shipping. In: Sausen, R., et al. (Eds.) International Conference on Transport, Atmosphere and Climate. European Commission, Oxford, pp. 202-207.

Lee, D.S., Pitari, G., Berntsen, T., Grewe, V., Gierens, K., Penner, J.E., Petzold, A Prather, M., Schumann, U., Bais, A., Iachetti, D., Lim, L.L., 2010. Transport impacts on atmosphere and climate: aviation. Atmospheric Environment 44, 4678-4734

Manne, A.S., Richels, R.G., 2001. An alternative approach to establishing trade-offs among greenhouse gases. Nature 410, 675-677.

Marais, K., Lukachko, S.P., Jun, M., Mahashabde, A., Waitz, I.A., 2008. Assessing the impact of aviation on climate. Meteorologische Zeitschrift 17, 157-172.

Montzka, S.A., Butler, J.H., Myers, R.C., Thompson, T.M., Swanson, T.H., Clarke, A.D., Lock, L.T., Elkins, J.W., 1996. Decline in the tropospheric abundance of halogen from halocarbons: implications for stratospheric ozone depletion. Science 272 1318-1322.

Murphy, J.M., Sexton, D.M.H., Barnett, D.N., Jones, G.S., Webb, M.J., Collins, M., 2004. Quantification of modelling uncertainties in a large ensemble of climate change simulations. Nature 430, 768-772.

Myhre, G., Stordal, F., 2001. On the tradeoff of the solar and thermal infrared radiative impact of contrails. Geophysical Research Letters 28, 3119-3122.

Naik, V., Mauzerall, D., Horowitz, L., Schwarzkopf, M.D., Ramaswamy, V. Oppenheimer, M., 2005. Net radiative forcing due to changes in regional emissions of tropospheric ozone precursors. Journal of Geophysical ResearchAtmospheres 110, D24306.

Naik, V., Mauzerall, D.L., Horowitz, L.W., Schwarzkopf, M.D., Ramaswamy, V. Oppenheimer, M., 2007. On the sensitivity of radiative forcing from biomass burning aerosols and ozone to emission location. Geophysical Research Letters 34, L03818.

Newman, P.A., Nash, E.R., Kawa, S.R., Montzka, S.A., Schauffler, S.M., 2006. When will the Antarctic ozone hole recover? Geophysical Research Letters 33, L12814.

Newman, P.A., Daniel, J.S., Waugh, D.W., Nash, E.R., 2007. A new formulation of equivalent effective stratospheric chlorine (EESC). Atmospheric Chemistry and Physics 7, 4537-4552.

Nordhaus, W.D., 2007. A review of the Stern Review on the economics of climate change. Journal of Economic Literature 45, 686-702. 
O'Neill, B.C., 2000. The jury is still out on global warming potentials. Climatic Change 44, 427-443.

O'Neill, B.C., 2003. Economics, natural science, and the costs of global warming potentials - an editorial comment. Climatic Change 58, 251-260.

Ponater, M., Pechtl, S., Sausen, R., Schumann, U., Huttig, G., 2006. Potential of the cryoplane technology to reduce aircraft climate impact: a state-of-the-art assessment. Atmospheric Environment 40, 6928-6944.

Prather, M.J., 1996. Time scales in atmospheric chemistry: theory, GWPs for $\mathrm{CH}_{4}$ and $\mathrm{CO}$, and runaway growth. Geophysical Research Letters 23, 2597-2600.

Prather, M.J., 2002. Lifetimes of atmospheric species: integrating environmental impacts. Geophysical Research Letters 29, 2063.

Rädel, G., Shine, K.P., 2008. Radiative forcing by persistent contrails and its dependence on cruise altitudes. Journal of Geophysical Research 113, D07105.

Reddy, M.S., Boucher, O., 2007. Climate impact of black carbon emitted from energy consumption in the world's regions. Geophysical Research Letters 34, L11802.

Rotmans, J., Den Elzen, M.G.J., 1992. A model-based approach to the calculation of global warming potentials (GWPs). International Journal of Climatology 12, 865-874.

Sausen, R., Isaksen, I., Grewe, V., Hauglustaine, D., Lee, D.S., Myhre, G., Kohler, M.O. Pitari, G., Schumann, U., Stordal, F., Zerefos, C., 2005. Aviation radiative forcing in 2000: an update on IPCC (1999). Meteorologische Zeitschrift 14, 555-561.

Sausen, R., Schumann, U., 2000. Estimates of the climate response to aircraft $\mathrm{CO}_{2}$ and $\mathrm{NO}_{x}$ emissions scenarios. Climatic Change 44, 27-58.

Schmalensee, R., 1993. Comparing greenhouse gases for policy purposes. Energy Journal 14, 245-256.

Schneider, S.H., Thompson, S.L., 1981. Atmospheric $\mathrm{CO}_{2}$ and climate - importance of the transient response. Journal of Geophysical Research-Oceans and Atmospheres 86, 3135-3147.

Schulz, M., Textor, C., Kinne, S., Balkanski, Y., Bauer, S., Berntsen, T., Berglen, T., Boucher, O., Dentener, F., Guibert, S., Isaksen, I.S.A., Iversen, T., Koch, D. Kirkevåg, A., Liu, X., Montanaro, V., Myhre, G., Penner, J.E., Pitari, G., Reddy, S., Seland, Ø., Stier, P., Takemura, T., 2006. Radiative forcing by aerosols as derived from the AeroCom present-day and pre-industrial simulations. Atmospheric Chemistry and Physics 6, 5225-5246.

Schumann, U., 2005. Formation, properties and climatic effects of contrails. Comptes Rendus Physique 6, 549-565.

Sherwood, S., 2007. Discounting and uncertainty: a non-economist's view. Climatic Change 80, 205-212.

Shine, K.P., Berntsen, T.K., Fuglestvedt, J.S., Bieltvedt Skeie, R., Stuber, N., 2007. Comparing the climate effect of emissions of short- and long-lived climate agents. Philosophical Transactions of the Royal Society A 365, 1903-1914.

Shine, K.P., Berntsen, T.K., Fuglestvedt, J.S., Sausen, R., 2005a. Scientific issues in the design of metrics for inclusion of oxides of nitrogen in global climate agreements. Proceedings of the National Academy of Sciences of the United States of America 102, 15768-15773.

Shine, K.P., Fuglestvedt, J.S., Hailemariam, K., Stuber, N., 2005b. Alternatives to the global warming potential for comparing climate impacts of emissions of greenhouse gases. Climatic Change 68, 281-302.

Shine, K.P., Fuglestvedt, J.S., Stuber, N., 2003. An Alternative to the Global Warming Potential for Comparing Climate Impacts of Emissions of Greenhouse Gases. Working Paper 2003:3. CICERO, Oslo, 17 pp.

Skodvin, T., Fuglestvedt, J.S., 1997. A comprehensive approach to climate change: political and scientific considerations. Ambio 26, 351-358.

Smith, S.J., 2003. The evaluation of greenhouse gas indices - an editorial comment. Climatic Change 58, 261-265.

Smith, S.J., Wigley, T.M.L., 2000. Global warming potentials: 1. Climatic implications of emissions reductions. Climatic Change 44, 445-457.

Solomon, S., Albritton, D.L., 1992. Time-dependent ozone depletion potentials for short-term and long-term forecasts. Nature 357, 33-37.

Stainforth, D.A., Aina, T., Christensen, C., Collins, M., Faull, N., Frame, D.J., Kettleborough, J.A., Knight, S., Martin, A., Murphy, J.M., Piani, C., Sexton, D. Smith, L.A., Spicer, R.A., Thorpe, A.J., Allen, M.R., 2005. Uncertainty in predictions of the climate response to rising levels of greenhouse gases. Nature 433, 403-406.
Stenke, A., Grewe, V., Pechtl, S., 2007. Do supersonic aircraft avoid contrails. Atmospheric Chemistry and Physics Discussions 7, 12927-12958.

Stern, N., 2007. The Economics of Climate Change: the Stern Review. Cambridge University Press, Cambridge, $692 \mathrm{pp}$.

Stevenson, D.S., Doherty, R.M., Sanderson, M.G., Collins, W.J., Johnson, C.E., Derwent, R.G., 2004. Radiative forcing from aircraft $\mathrm{NO}_{x}$ emissions: mechanisms and seasonal dependence. Journal of Geophysical Research-Atmospheres 109, D17307.

Stuber, N., Forster, P., Rädel, G., Shine, K., 2006. The importance of the diurnal and annual cycle of air traffic for contrail radiative forcing. Nature 441, 864-867.

Svensson, F., Hasselrot, A., Moldanova, J., 2004. Reduced environmental impact by lowered cruise altitude for liquid hydrogen-fuelled aircraft. Aerospace Science and Technology 8, 307-320.

Tanaka, K., O’Neill, B.C., Rokityanskiy, D., Obersteiner, M., Tol, R.S.J. Evaluating global warming potentials with historical temperature. Climatic Change, in press.

Tol, R.S.J., Berntsen, T.K., O’Neill, B.C., Fuglestvedt, J.S., Shine, K.P., Balkanski, Y., Makra, L., 2008. Metrics for Aggregating the Climate Effect of Different Emissions: a Unifying Framework. The Economic and Social Research Institute, Dublin. http://www.esri.ie/UserFiles/publications/20080924144712/WP257.pdf ESRI Working Paper 257.

Uherek, E., Halenka, T., Berntsen, T., Balkanski, Y., Borken, Y., Gauss, M., Hoor, P., Juda-Rezler, K., Lelieveld, J., Melas, D., Rypdal, K., Sausen, R., Schmid, S., 2010. Transport impacts on atmosphere and climate: land transport. Atmospheric Environment 11, 4772-4816.

UNEP, 2007. Environmental Effects of Ozone Depletion: 2006 Assessment. United Nations Environment Programme, Nairobi.

USEPA, 2000. Guidelines for Preparing Economic Analyses. United States Environmental Protection Agency. Available from: http://yosemite.epa.gov/ee/epa/eed. nsf/webpages/Guidelines.html.

van Vuuren, D.P., Weyant, J., de la Chesnaye, F., 2006. Multi-gas scenarios to stabilize radiative forcing. Energy Economics 28, 102-120.

Weitzman, M.L., 2007. Review of the Stern Review on the economics of climate change. Journal of Economic Literature 45, 703-724.

Wigley, T.M.L., Ammann, C.M., Santer, B.D., Raper, S.C.B., 2005. Effect of climate sensitivity on the response to volcanic forcing. Journal of Geophysical ResearchAtmospheres 110, D09107.

Wigley, T.M.L., Raper, S.C.B., 2001. Interpretation of high projections for globalmean warming. Science 293, 451-454.

Wild, O., Prather, M.J., Akimoto, H., 2001. Indirect long-term global radiative cooling from $\mathrm{NO}_{x}$ emissions. Geophysical Research Letters 28, 1719-1722.

Wit, R.C.N., Boon, B.H., van Velzen, A., Cames, M., Deuber, O., Lee, D.S., 2005. Giving Wings to Emission Trading. Inclusion of Aviation Under the European Emission Trading System (ETS): Design and Impacts. CE Delft, The Netherlands.

WMO, 1992. Scientific Assessment of Ozone Depletion: 1991: Global Ozone Research and Monitoring Project Report No 25. World Meteorological Organization, Geneva, Switz.

WMO, 1999. Scientific Assessment of Ozone Depletion: 1998: Global Ozone Research and Monitoring Project Report No 44. World Meteorological Organization, Geneva, Switz.

WMO, 2003. Scientific Assessment of Ozone Depletion: 2002: Global Ozone Research and Monitoring Project Report No 47. World Meteorological Organization, Geneva, Switz.

WMO, 2007. Scientific Assessment of Ozone Depletion: 2006: Global Ozone Research and Monitoring Project Report No 50. World Meteorological Organization, Geneva, Switz.

Wuebbles, D.J., 1983. Chlorocarbon emission scenarios - potential impact on stratospheric ozone. Journal of Geophysical Research-Oceans and Atmospheres $88,1433-1443$.

Wuebbles, D.J., Yang, H., Herman, R., 2008. Climate Metrics and Aviation: Analysis of Current Understanding and Uncertainties (Subject Specific White Paper). Aviation Climate Change Research Initiative (ACCRI). Available from: www.faa. gov/about/office_org/headquarters_offices/aep/aviation_climate/media/ACCRI_ SSWP_VIII_Wuebbles.pdf. 